\title{
Prediction of Tensile Behaviors of L-DED 316 Stainless Steel parts using Machine Learning
}

Israt Zarin Era

ie0005@mix.wvu.edu

Follow this and additional works at: https://researchrepository.wvu.edu/etd

Part of the Industrial Engineering Commons, and the Manufacturing Commons

\section{Recommended Citation}

Era, Israt Zarin, "Prediction of Tensile Behaviors of L-DED 316 Stainless Steel parts using Machine Learning" (2021). Graduate Theses, Dissertations, and Problem Reports. 8274.

https://researchrepository.wvu.edu/etd/8274

This Thesis is protected by copyright and/or related rights. It has been brought to you by the The Research Repository @ WVU with permission from the rights-holder(s). You are free to use this Thesis in any way that is permitted by the copyright and related rights legislation that applies to your use. For other uses you must obtain permission from the rights-holder(s) directly, unless additional rights are indicated by a Creative Commons license in the record and/ or on the work itself. This Thesis has been accepted for inclusion in WVU Graduate Theses, Dissertations, and Problem Reports collection by an authorized administrator of The Research Repository @ WVU. For more information, please contact researchrepository@mail.wvu.edu. 
Prediction of Tensile Behaviors of L-DED 316 Stainless Steel parts using Machine Learning

Israt Zarin Era

Follow this and additional works at: https://researchrepository.wvu.edu/etd

Part of the Industrial Engineering Commons, and the Manufacturing Commons 


\title{
Prediction of Tensile Behaviors of L-DED 316 Stainless Steel parts using Machine Learning
}

\author{
Israt Zarin Era
}

\begin{abstract}
Thesis submitted to the College of Engineering \& Mineral Resources at West Virginia University in partial fulfillment of the requirements for the degree of
\end{abstract}

\author{
Master of Science \\ In \\ Industrial Engineering \\ Zhichao Liu, Ph.D, Chair \\ Kenneth R. Currie, Ph.D., P.E. \\ Thorsten Wuest, Ph.D.
}

Department of Industrial and Management Systems Engineering

\author{
Morgantown, West Virginia
}

July 2021

Keywords: Machine learning, Directed Energy Deposition, XGBoost, Ridge Regression, Bootstrap

Copyright 2021 Israt Zarin Era 


\section{ABSTRACT \\ Prediction of Tensile Behaviors of L-DED 316 Stainless Steel parts using Machine Learning}

\section{Israt Zarin Era}

Directed energy deposition (DED) is a rising field in the arena of metal additive manufacturing and has extensive applications in aerospace, medical and rapid prototyping. The process parameters, such as laser power, scanning speed and specimen height, play a great deal in controlling and affecting the properties of DED fabricated parts. Nevertheless, both experimental and simulation methods have shown constraints and limited ability to generate accurate and efficient computational predictions on the correlations between the process parameters and the final part quality. In this work, a data driven machine learning model XGBoost has been built and applied to predict the tensile behaviors of the stainless steel 316 parts by DED with variation in process parameters i.e. laser power, scanning speed, layer height and energy density. For the validation purpose, molten pool temperature data has been provided to the model and it was able to predict the molten pool temperature successfully with a very high accuracy. After the tensile testing, the model was able to predict the tensile properties i.e. yield strength, elongation (\%) and ultimate tensile strength of the fabricated parts with a limited size of training data and to compute the significance of the factors affecting the part quality. Performance of the model was then compared with ridge regression and XGBoost outperformed ridge regression. 


\section{ACKOWLEDGEMENT}

I would like to thank my research supervisor Dr. Zhichao Liu for his unparallel support and guidance during the course of work. I also thank him for encouraging and funding me for the IISE Annual Conference and Expo 2021.

I am grateful to Almighty, my parents and my husband for their continuous support and blessing which helped to complete my M.Sc. degree successfully. A special thanks to Sajid Mahmud, who initially helped me with the resource material of machine learning. I wish to thank my colleagues and friends who supported me during my endeavors. 


\section{$\underline{\text { Table of Contents }}$}

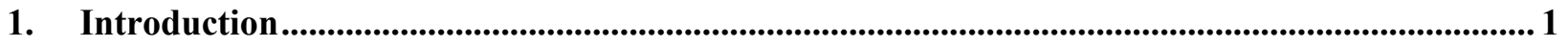

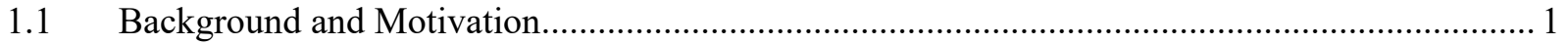

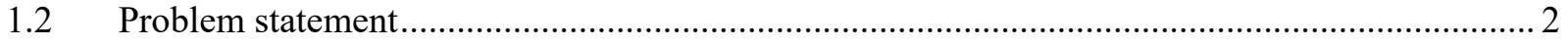

2. Literature Review ............................................................................................................................. 3

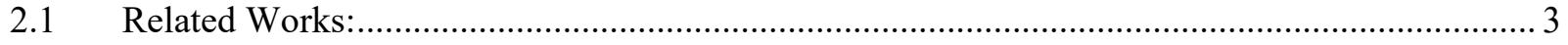

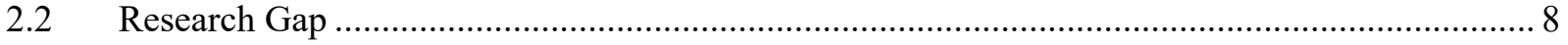

2.3 Challenges and Proposed Solution:..................................................................................... 9

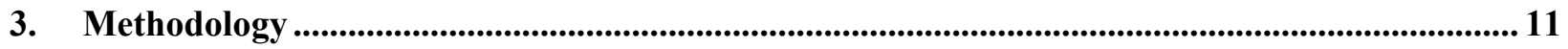

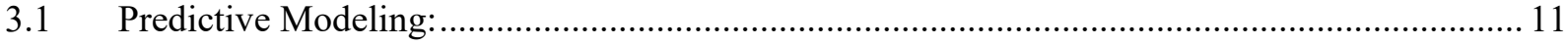

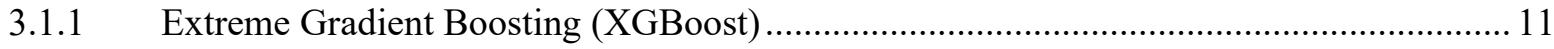

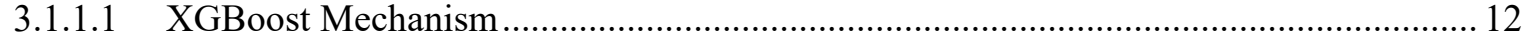

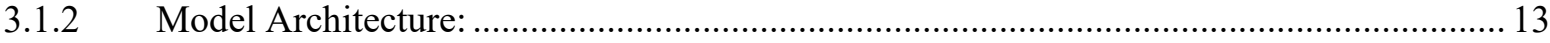

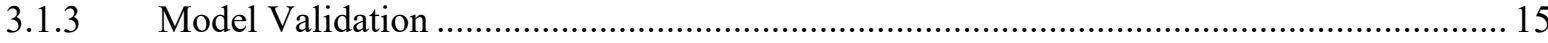

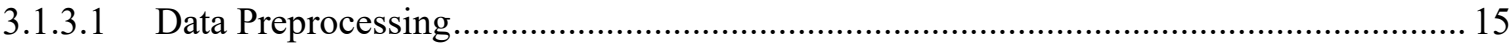

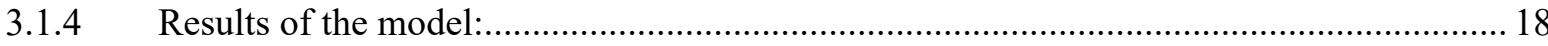

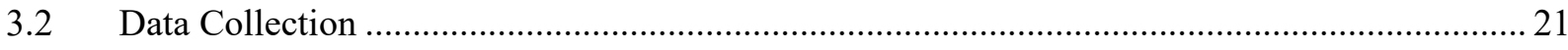

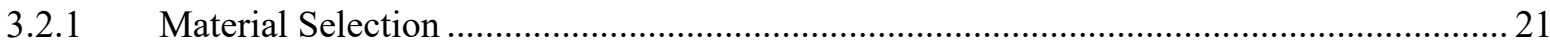

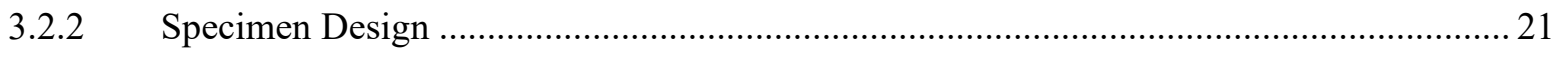

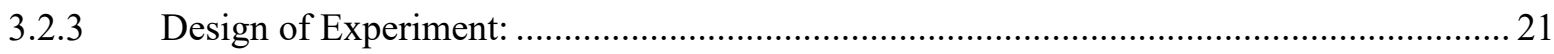

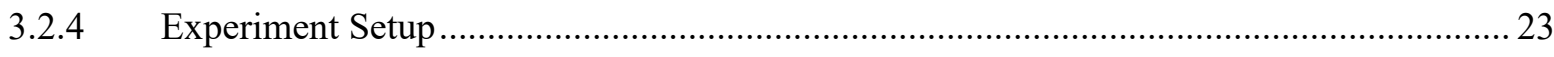

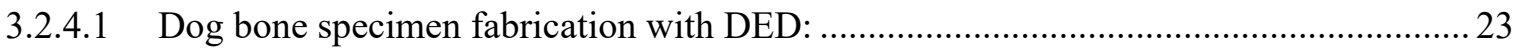

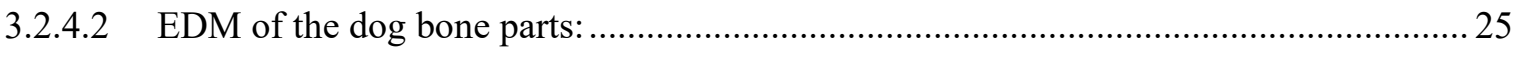

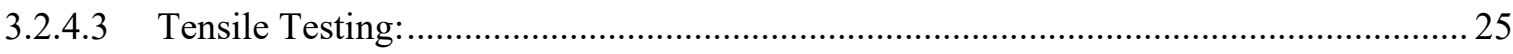

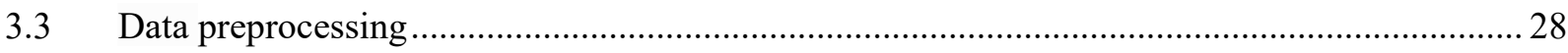

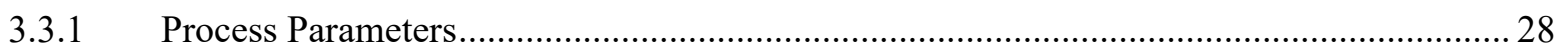

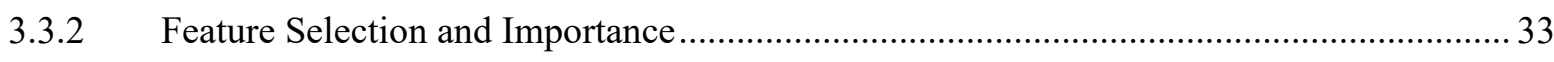

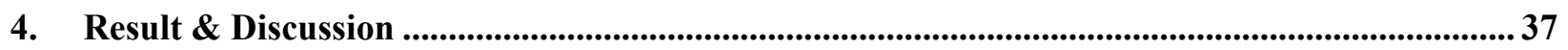

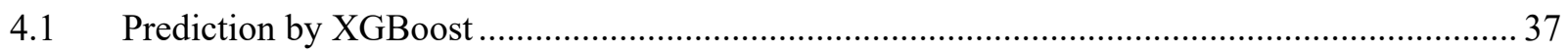

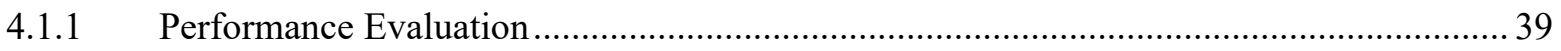

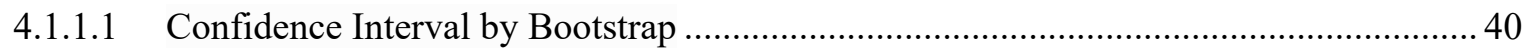

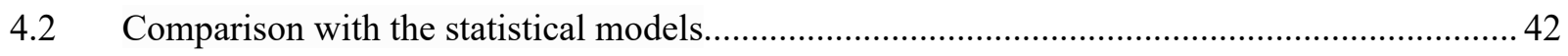

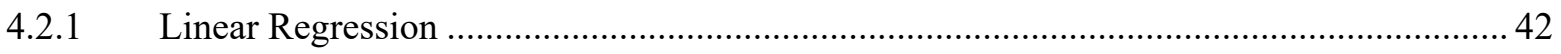


4.2.1.1 Regression Analysis: Elongation (\%) versus Power (W), Scanning Speed (mm/sec), Layer Height $(\mathrm{mm})$, Energy Density $\left(\mathrm{J} / \mathrm{mm}^{2}\right)$

4.2.1.2 Regression Analysis: UTS (MPa) versus Power (W), Scanning Speed (mm/sec), layer Height (mm), Energy Density $\left(\mathrm{J} / \mathrm{mm}^{2}\right)$

4.2.1.3 Regression Analysis: Yield Strength (MPa) versus Power (W), Scanning Speed

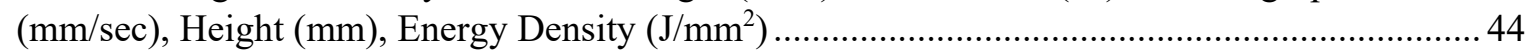

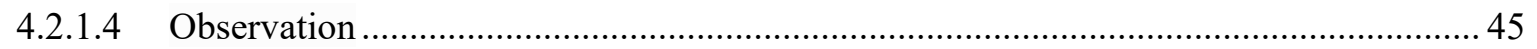

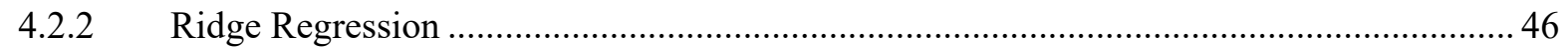

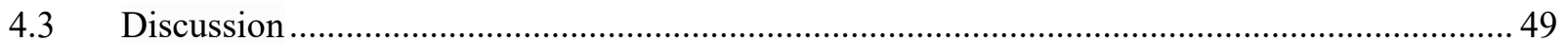

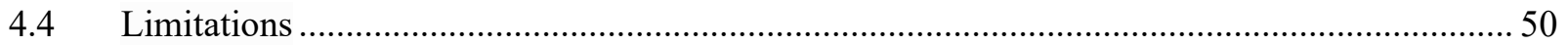

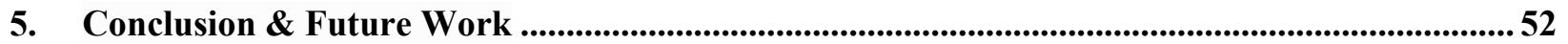

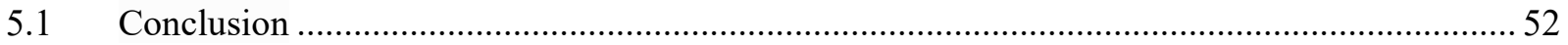

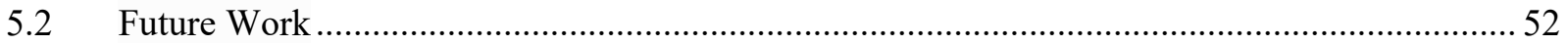

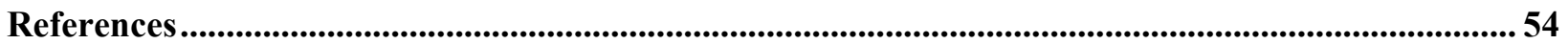




\section{Lists of Tables}

Table 1: Summary of Prediction Models using Machine learning

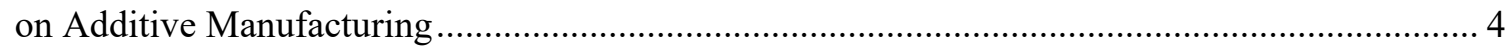

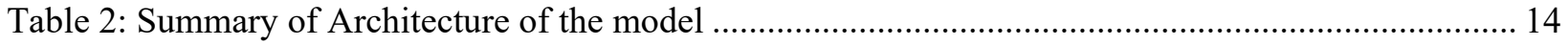

Table 3: Design of Experiment of molten pool temperature ................................................................ 16

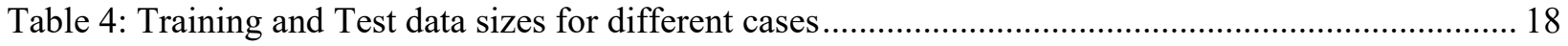

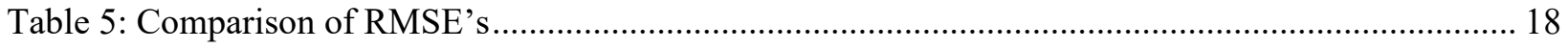

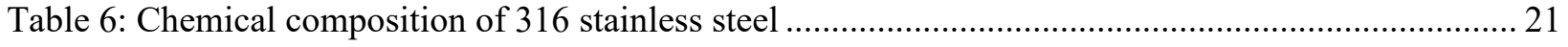

Table 7: Design of Experiment of Dog bone specimens..................................................................... 22

Table 8: Design of Experiment with groups with parameters.............................................................. 22

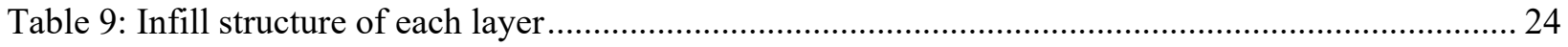

Table 10: Comparison between the mechanical properties of printed and raw $316 \mathrm{SS}$........................... 27

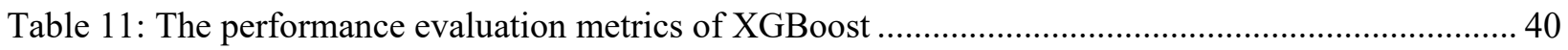

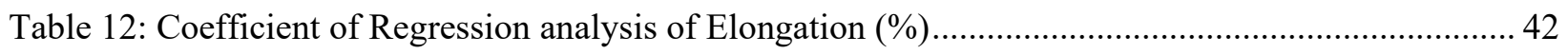

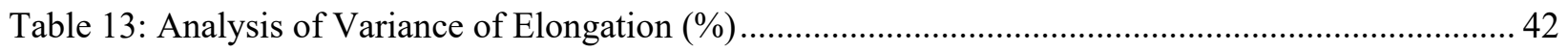

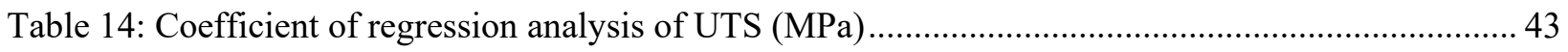

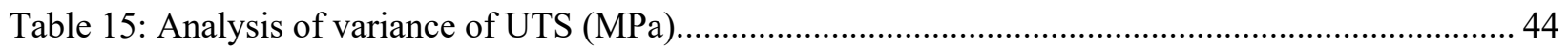

Table 16: Coefficient of Regression analysis of Yield Strength (MPa) .................................................. 44

Table 17: Analysis of variance of Yield Strength (MPa) ....................................................................... 45

Table 18: The performance evaluation metrics of Ridge Regression .................................................... 48 


\section{$\underline{\text { List of Illustrations }}$}

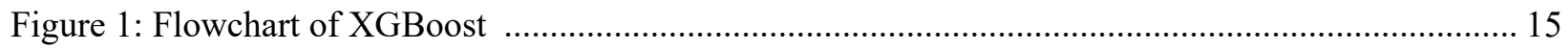

Figure 2: The molten pool temperature of Experiment 1 (laser power: $250 \mathrm{~W}$, scanning speed:11 mm/s) 17

Figure 3: Molten pool temperature of eight layers of the specimen of

experiment 1 after removing the dwelling temperature data

Figure 4: Predicted and observed molten pool temperature of experiment 1

(laser power: $250 \mathrm{~W}$, scanning speed: $11 \mathrm{~mm} / \mathrm{s}$ )

Figure 5: Predicted and observed molten pool temperature of experiment 7

(laser power: $350 \mathrm{~W}$, scanning speed: $7 \mathrm{~mm} / \mathrm{s}$ )

Figure 6: Predicted and observed molten pool temperature of experiment 21

(laser power: $250 \mathrm{~W}$, scanning speed: $3 \mathrm{~mm} / \mathrm{s}$ )

Figure 7: Predicted and observed molten pool temperature of experiment 25

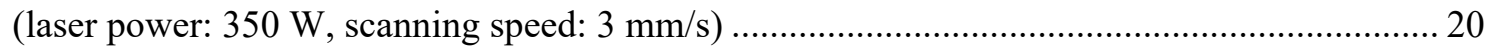

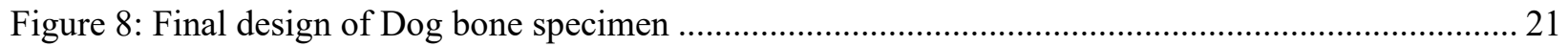

Figure 9: Schematic diagram of $\mathrm{AMBIT}^{\mathrm{TM}}$ laser based

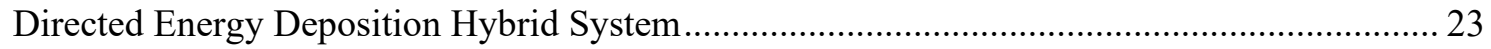

Figure 10: 3D printed 316 SS Dog bone specimens by L-DED process .............................................. 24

Figure 11: Electric Discharge Machining and the final dog bone specimen .........................................2 25

Figure 12: Universal testing Machine and dog bone specimen after failure .......................................... 26

Figure 13: Yield Strength from the stress-strain curve graph (for sample \#6) ........................................ 26

Figure 14: Yield Strength (MPa) VS. Laser Power (W) for $11 \mathrm{~mm} / \mathrm{sec}$ scanning speed.......................... 28

Figure 15: Elongation (\%) VS. Laser Power (W) for $11 \mathrm{~mm} / \mathrm{sec}$ scanning speed................................... 29

Figure 16: UTS (MPa) VS. Laser Power (W) for $11 \mathrm{~mm} / \mathrm{sec}$ scanning speed ........................................ 29

Figure 17: Yield strength (MPa) VS. energy density $\left(\mathrm{J} / \mathrm{mm}^{2}\right)$ for $11 \mathrm{~mm} / \mathrm{sec}$ scanning speed.................. 30

Figure 18: Elongation (\%) VS. energy density $\left(\mathrm{J} / \mathrm{mm}^{2}\right)$ for $11 \mathrm{~mm} / \mathrm{sec}$ scanning speed ........................ 30

Figure 19: Ultimate tensile strength (MPa) VS. energy density $\left(\mathrm{J} / \mathrm{mm}^{2}\right)$ for $11 \mathrm{~mm} / \mathrm{sec}$ scanning speed .. 31

Figure 20: Yield Strength (MPa) VS. Layer Height $(\mathrm{mm})$ for $11 \mathrm{~mm} / \mathrm{sec}$ scanning speed ....................... 31

Figure 21: Elongation (\%) VS. Layer Height (mm) for $11 \mathrm{~mm} / \mathrm{sec}$ scanning speed ............................... 32

Figure 22: Ultimate Tensile Strength (MPa) VS. Layer Height (mm) for $11 \mathrm{~mm} / \mathrm{sec}$ scanning speed ..... 32

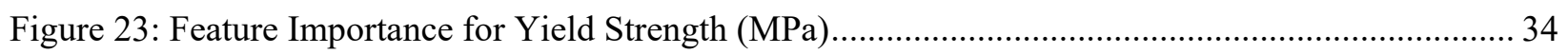

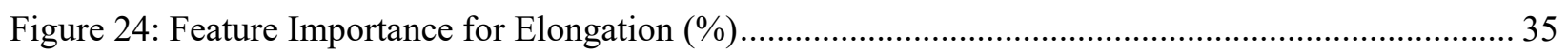

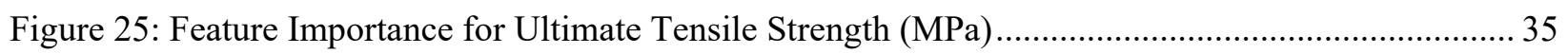

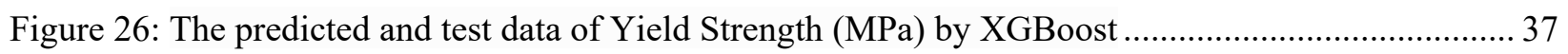

Figure 27: The predicted and test data of Elongation (\%) by XGBoost ................................................. 38 
Figure 28: The predicted and test data for UTS (MPa) by XGBoost..................................................... 38

Figure 29: The histogram of distribution of RMSE of yield strength (MPa) by bootstrap...................... 41

Figure 30: The histogram of distribution of RMSE of ultimate tensile strength (MPa) by bootstrap ........ 42

Figure 31: The histogram of distribution of RMSE of elongation (\%) by bootstrap ............................... 42

Figure 32: The predicted and test data for Yield Strength (MPa) by Ridge Regression .......................... 47

Figure 33: The predicted and test data for Elongation (\%) by Ridge Regression .................................... 48

Figure 34: The predicted and test data for UTS (MPa) by Ridge Regression. ........................................ 48 


\section{Introduction}

\subsection{Background and Motivation}

The world is moving from Subtractive Manufacturing to the Additive Manufacturing popularly called 3D printing technology. It has been declared as the "third industrial revolution" by Economist magazine [April-2012], (Jyoti Mazumder, 2015). Additive manufacturing possesses a domininance over other convemtional manufacturing processes, exceling in printing complex shapes avoiding different traditional processes, such as expensive tooling, dies, or casting molds. (Khairallah et. al, 2017). Our work focuses on one such type of AM process, known as directed energy deposition (DED). In DED systems, At the presence of inert gas, there are a strenuous heat source and a flow of raw material, both concentrating at a common focal point. The heat concentrated at a particular point melts the both the substrate and the powder creating melt pool around that spot. (Dass et. al, 2019). DED systems can be classified on basis of types of feeds and Energy Sources. According to feed type, there are two kinds of DED systems: Powder feed and Wire feed.

Extensive studies have been done on powder based DED system and it is the most used metal DED technique. DED process is able to print complete solid parts with highly controllable microstructures. This process can produce functionally graded components with different compositions along three axes. (Froes et al, 2019) The combined strength of DED processes enables it to be utilized to develop, repair, overhaul and modernize of new materials, improve the life of injection molding or die casting dies filling wear resistant alloys where there is high possibilties of high wear.(Gibson et al., 2015).

Recently, DED is getting popular for extensive usage in Aerospace, Defense, Automobile and Biomedical industries. (Froes et al., 2019). Despite the advanteages of DED processes are prone to poor resolution and rough surface finish. It is difficult to attain an accuracy more than $0.25 \mathrm{~mm}$ and a surface roughness of less than $25 \mu \mathrm{m}$. (Gibson et al., 2015) Despite the advantages over other AM processes, DED process is prone to pores and cracks (Khanzadeh et al. 2019) which can compromise the quality of the final product. Entrapped gas, lack of fusion, rapid solidification and incomplete powder melting are identified as the possible causes of the defective microstructures (Shamsaei et al.,2015). Slower build speed is another limitation. Due to slower deposition rate, the build speed is lengthier. To achieve better accuracies, small beam sizes and rapid deposition rates are required. Rapid deposition rates result in degradation of resolution and surface finish. Variations in laser power and scan rate to achieve etter accuracies or deposition rates may also affect the microstructures of the depositedb components, and thus finding an optimum deposition condition requires tradeoffs between build speed, accuracy, and microstructure. (Gibson et al., 2015) 


\subsection{Problem statement}

Evaluation of mechanical properties of printed parts have been proved to be befitting of understanding the relationship between the parameters and print quality of fabricated parts in previous studies (Zhang et al., 2017). Tensile strength is a fine indicator of mechanical behavior of a particular material. Tensile tests are used to determine how materials will behave under tension load and to verify that materials adhere to minimum strength and elongation requirements (Dass et al., 2019). The selection of process parameters is always critical to the quality of the manufactured product. Lots of efforts have been made on investigating the effects of processing parameters on output quality from experiential perspective. The tensile strength of printed parts is one of the quality-assuring mechanical properties which is dependent on the DED process parameters. To establish the correlation among the process parameters and mechanical properties, both experiment-based models and simulation modeling are prominently employed by the researchers. But both experiment-based and simulation-based models appear to have significant setbacks.

Over the period of numerous studies, machine learning algorithms have become the leading tools to use data driven predictive modeling. But the area of predicting tensile strength of L-DED specimens is still not well explored by ML users. The purpose of our work is to investigate and predict the correlation among tensile strength of the printed parts and the process parameters of Laser Based Directed Energy Deposition using machine learning. The goal is to address the challenges arising from both experimentation and modeling. The intended research will be able to impact the field of Additive Manufacturing by using Machine learning tools. The proposed ML model should be able to predict physical properties of the fabricated specimens of L-DED which can contribute to reduce process time, material wastes and expenses concerning them. Dependence on trial and error experimental methods can be lessened greatly and they can be replaced with predictive data-driven models for future validations. 


\section{Literature Review}

\subsection{Related Works:}

An enriched literature is available on data driven models on various AM process parameters. Zhang et. al, 2020 developed a data-driven predictive model using two machine learning algorithms i.e. extreme gradient boosting (XGBoost) and long short-term memory (LSTM) to estimate the melt pool temperature during DED with high accuracy. The same authors (2019) introduced a Data-Driven predictive modeling approach to estimate the tensile strength of polymers fabricated by the cooperative 3D printing process. The authors used Ensemble learning that combines multiple learning algorithms including Lasso, Support Vector Regression and XGBoost and compare the results with Ridge Regression. They claimed the ensemble learning method outperformed the linear regression mode. For instances, the RMSEs of the ensemble learning model are 3.627, 4.339, and 2.648, while the RMSEs of the linear regression model are $5.28,4.19$, and 4.76 when $50 \%, 70 \%$, and $90 \%$ of the total data are used as training data respectively. Wu et. al, 2019 presented an ensemble learning-based approach to surface roughness prediction in FFF processes. To improve computational efficiency and to avoid overfitting, a subset (40) of the features was selected using RF based on feature importance. The ensemble learning algorithm combined six different machine learning algorithms, including RF, AdaBoost, CART, SVR, RR, and RVFL network. The authors claimed the performance of the ensemble approach outperforms the individual base learners based on RMSE and RE. Luke Scime et. al 2019 and Sarah J. Wolff et. al, 2019 worked on Inconel 718 material for flaw formation in Laser Based Powder Bed Fusion using BoW and SIFT method and experimentally validated prediction of thermal history and microhardness in DED process by a computational thermo-fluid dynamics (CtFD) model respectively. Both works demonstrated that the proposed inexpensive and welltested computational frame works can generate a large amount of high-quality prediction data, including temperature field, velocity field, melt pool dimensions, dilution, heating and cooling rates, solidification parameters, and microhardness. Li et. al, 2019 modeled the degradation and remaining useful life prediction of Aircraft Engines using particle swarm optimization (PSO) and sequential quadratic optimization (SQP) based Ensemble Learning which combines seven machine learning algorithms of different types, including RFs, Classification and Regression tree (CART), Recurrent Neural Networks (RNN), Autoregressive (AR) model, Adaptive networkbased fuzzy inference system (ANFIS), Relevance vector machine (RVM), and Elastic net (EN). The authors claimed their model outperformed base learners significantly based on S-score, RE, and RMSE values.

Fabrizia Caiazzo et. al, 2018 took a machine learning approach based on Artificial Neural Networks to find the correlation between the laser metal deposition process parameters and the output geometrical parameters of the deposited metal trace produced by laser direct metal deposition on 5-mm-thick 2024 aluminum alloy plates. Mojtaba Khanzadeh et. al, 2018 performed porosity prediction method using morphological characteristics of the melt pool boundary by Supervised Learning using Decision Tree (DT), K-Nearest Neighbor (KNN), Support Vector Machine (SVM), Linear Discriminant Analysis (LDA), and Quadratic Discriminant 
Analysis (QDA)). Dazhong Wu et. al, 2018 used RFs, SVR, RR, and LASSO to predict Surface roughness in fused deposition modelling. Recurrent Neural Network (RNN) is applied in prediction of thermal history in directed energy deposition processes by Mojtaba Mozaffar et. al, 2018. The authors mentioned considering that the accuracy of the model can be further improved by increasing training epochs and geometry types included in the database.

Wu et. al, 2017 also applied RF, ANN, SVR in order to perform Tool wear prediction. Batut et. al, 2017 performed temperature prediction in direct metal deposition by a numerical and an analytical model. A hybrid artificial neural network (ANN) with genetic algorithm approach (GA) is constructed to predict of tensile properties by Hayes et. al, 2017. Venkata Rao, 2016 executed optimization of fused deposition modeling process by teaching-learning-based optimization (TLBO) algorithm and non-dominated Sorting TLBO (NSTLBO) algorithm. Hansel et. al, 2016 applied Taguchi method to predict optimum deposition conditions of tensile strength of typical metal material by AM.

Onwubolu et. al, 2014 employed Finite Element Analysis Method for Fused Deposition Modelling Process. The process parameters and the experimental results were through a hybrid GMDH-GA system resulted predicted output, which found correlated very closely with the experimental values. Garg et. al, 2014 introduced an ensemble method of genetic programming (GP) and artificial neural network for formulating a model for predicting the wear strength of the FDM fabricated prototype. The results indicated that ensemble model have performed better than that of the standardized GP, which used to be performed by experts for optimizing the performance of the FDM process before. Cortez et. al, 2013 introduced Sensitivity analysis to open black box data mining models using Synthesized data and real- life scenarios. Benardos et. al, 2002 performed prediction of surface roughness using neural networks and Taguchi's design of experiments.

Table 1: Summary of Prediction Models using Machine learning on Additive Manufacturing

\begin{tabular}{|c|c|c|c|c|}
\hline Metrics & Model Input & Model output & Material & Reference \\
\hline $\begin{array}{c}\text { XGBoost and } \\
\text { LSTM Neural } \\
\text { Network }\end{array}$ & $\begin{array}{l}\text { - } \text { Power } \\
\text { - Scanning Speed } \\
\text { - Specimen } \\
\text { Height }\end{array}$ & $\begin{array}{l}\text { Molten Pool } \\
\text { Temperature }\end{array}$ & Inconel 718 & $\begin{array}{c}\text { Zhang et. al } \\
\text { (2020) }\end{array}$ \\
\hline $\begin{array}{l}\text { Ensemble } \\
\text { learning with } \\
\text { Lasso, SVR, } \\
\text { and extreme }\end{array}$ & $\begin{array}{l}\text { - Angle of incline } \\
\text { - Overlapping } \\
\text { length } \\
\text { - Number of } \\
\text { shells }\end{array}$ & Tensile Strength & $\begin{array}{c}\text { Polylactide } \\
\text { (PLA) }\end{array}$ & $\begin{array}{c}\text { Zhang et. al } \\
\text { (2019) }\end{array}$ \\
\hline
\end{tabular}




\begin{tabular}{|c|c|c|c|c|}
\hline $\begin{array}{l}\text { gradient } \\
\text { XGBoost }\end{array}$ & & & & \\
\hline $\begin{array}{l}\text { Microstructures } \\
\text { and tensile } \\
\text { properties }\end{array}$ & $\begin{array}{ll}\text { - } & \text { Powder feed } \\
& \text { rate } \\
\text { - } & \text { Laser Power } \\
\text { - } & \text { Scanning Speed } \\
\text { - } & \text { Laser Spot } \\
\text { - } & \text { Diameter } \\
\text { - } & \text { Carrier Gas }\end{array}$ & $\begin{array}{ll}\text { - } & \text { Microstructure } \\
\text { - } & \text { Tensile } \\
& \text { Strength }\end{array}$ & Inconel 718 & Fu et. al (2016) \\
\hline Taguchi method & $\begin{array}{ll}\text { - } & \text { Laser Power } \\
\text { - } & \text { Feed Speed } \\
\text { - } & \text { Powder } \\
\text { - } & \text { Flow } \\
\text { - } & \text { Carrier Gas }\end{array}$ & $\begin{array}{ll}\text { - } & \text { Tensile } \\
& \text { Strength } \\
\text { - } & \text { Yield Strength } \\
\text { - } & \text { Elongation }\end{array}$ & $\begin{array}{c}\text { - } \mathrm{X} 2 \mathrm{CrNi} \\
\mathrm{Mo} 17- \\
12-2 \\
-\quad \text { Inconel } \\
625\end{array}$ & $\begin{array}{l}\text { Hansel et. al } \\
\text { (2016) }\end{array}$ \\
\hline $\begin{array}{c}\text { Finite Element } \\
\text { Analysis }\end{array}$ & $\begin{array}{ll}\text { - } & \text { Layer } \\
& \text { Thickness } \\
\text { - } & \text { Part Orientation } \\
\text { - } & \text { Raster Angle } \\
\text { - } & \text { Raster Width } \\
\text { - } & \text { Air Gap }\end{array}$ & $\begin{array}{ll}- & \text { Tensile } \\
& \text { Strength }\end{array}$ & ABS material & $\begin{array}{c}\text { Onwubolu et. al } \\
\text { (2014) }\end{array}$ \\
\hline $\begin{array}{c}\text { A hybrid } \\
\text { artificial neural } \\
\text { network (ANN) } \\
\text { p genetic } \\
\text { algorithm } \\
\text { approach (GA) }\end{array}$ & $\begin{array}{ll}\text { - } & \text { AM- } \alpha+\beta \text { stress } \\
& \text { relieved } \\
\text { - } & \text { AM- } \alpha+\beta \text { HIP } \\
\text { - } & \text { AM- } \alpha+\beta \\
& \text { annealed }\end{array}$ & $\begin{array}{l}\text { - Yield Strength } \\
\text { - Ultimate } \\
\text { Tensile } \\
\text { Strength }\end{array}$ & Ti-6Al-4V & $\begin{array}{l}\text { Hayes et. al } \\
\text { (2017) }\end{array}$ \\
\hline $\begin{array}{c}\text { Ensemble } \\
\text { Learning with } \\
\text { RF, AdaBoost, } \\
\text { (CART), } \\
\text { (SVR), (RR), } \\
\text { and (RVFL) }\end{array}$ & $\begin{array}{ll}\text { - } & \text { Layer } \\
& \text { Thickness } \\
\text { - } & \text { Extruder } \\
& \text { Temperature } \\
\text { - } & \text { Print } \\
\text { Speed/Extrusion } \\
\text { Speed }\end{array}$ & - Roughness & $\begin{array}{l}\text { Polylactic Acid } \\
\text { (PLA) }\end{array}$ & Wu et. al (2019) \\
\hline $\begin{array}{l}\text { Neural networks } \\
\text { and Taguchi's } \\
\text { design of } \\
\text { experiments }\end{array}$ & $\begin{array}{ll}\text { - } & \text { Feed Rate } \\
\text { - } & \text { Cutting Speed } \\
& \text { Tool Wear } \\
\text { - } & \text { Cutting Fluid }\end{array}$ & $\begin{array}{ll}\text { - } & \text { Surface } \\
& \text { Roughness }\end{array}$ & $\begin{array}{l}\text { Series } 2 \\
\text { Aluminum } \\
\text { alloy }\end{array}$ & $\begin{array}{c}\text { Benardos et. al } \\
\quad(2002)\end{array}$ \\
\hline
\end{tabular}




\begin{tabular}{|c|c|c|c|c|}
\hline & $\begin{array}{ll}\text { - } & \text { Engagement } \\
\text { - } & \text { Cutting Depth }\end{array}$ & & & \\
\hline BoW and SIFT & $\begin{array}{ll}\text { - } & \text { Beam Power } \\
\text { - } & \text { Beam Velocity } \\
\end{array}$ & $\begin{array}{l}\text { In situ melt pool } \\
\text { temperature }\end{array}$ & $\begin{array}{c}\text { Inconel } 718 \\
\text { material }\end{array}$ & $\begin{array}{l}\text { Scime et. al } \\
(2019)\end{array}$ \\
\hline $\begin{array}{c}\text { Finite Element } \\
\text { Analysis }\end{array}$ & $\begin{array}{ll}\text { - } & \text { Laser Power } \\
\text { - } & \text { Scanning Speed }\end{array}$ & Surface Temperature & Ti6Al4V & Batut et. al (2017) \\
\hline $\begin{array}{l}\text { Computational } \\
\text { thermo-fluid } \\
\text { dynamics model }\end{array}$ & $\begin{array}{ll}\text { - } & \text { Clad Height } \\
\text { - } & \text { Powder Flow } \\
\text { - } & \text { Laser Power }\end{array}$ & $\begin{array}{ll}\text { - } & \text { Dilution } \\
\text { - } & \text { Microstructure } \\
\text { - } & \text { Microhardness } \\
\text { - } & \text { Melt Pool } \\
& \text { Geometry } \\
\text { - } & \text { Temperature } \\
\end{array}$ & $\begin{array}{l}\text { Inconel } 718 \text { on } \\
\text { carbon steel }\end{array}$ & $\begin{array}{l}\text { Wolff et. al } \\
\text { (2019) }\end{array}$ \\
\hline $\begin{array}{c}\text { Recurrent } \\
\text { neural networks }\end{array}$ & 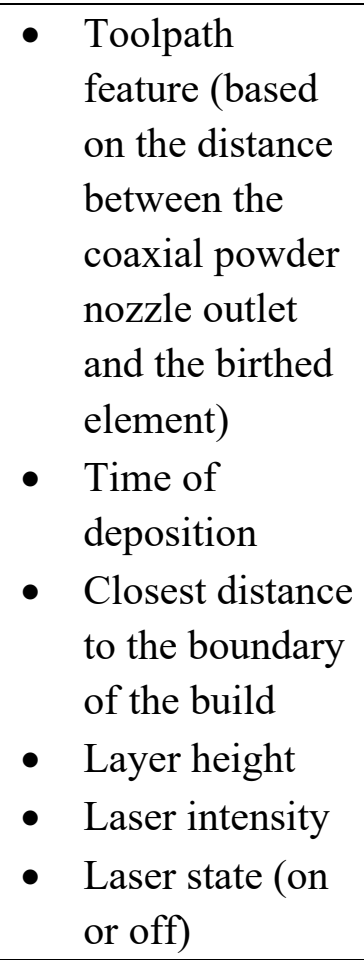 & $\begin{array}{l}\text { Melt Pool } \\
\text { Temperature }\end{array}$ & $\begin{array}{c}\text { Stainless steel } \\
316 \mathrm{~L}\end{array}$ & $\begin{array}{l}\text { Mozaffar et. al } \\
\text { (2018) }\end{array}$ \\
\hline $\begin{array}{l}\text { An Ensemble } \\
\text { Approach }\end{array}$ & $\begin{array}{ll}\text { - } & \text { Layer thickness } \\
& \text { Orientation } \\
\text { - } & \text { Raster Angle } \\
\text { - } & \text { Raster width } \\
\text { - } & \text { Air gap }\end{array}$ & Wear strength. & $\begin{array}{l}\text { Material not } \\
\text { mentioned }\end{array}$ & Garg et. al (2014) \\
\hline RF, ANN, SVR & - Cutting Force & Tool wear & $\begin{array}{c}\text { High-speed } \\
\text { steel and } \\
\text { stainless steel }\end{array}$ & Wu et. al (2017) \\
\hline
\end{tabular}




\begin{tabular}{|c|c|c|c|c|}
\hline $\begin{array}{l}\text { Optimization of } \\
\text { fused deposition } \\
\text { modeling } \\
\text { process }\end{array}$ & $\begin{array}{ll}\text { - } & \text { Layer } \\
& \text { Thickness } \\
& \text { Orientation } \\
\text { - } & \text { Raster Angle } \\
\text { - } & \text { Raster Width } \\
\text { - } & \text { Air Gap } \\
\end{array}$ & Wear & $\begin{array}{l}\text { Acrylonitrile } \\
\text { butadiene } \\
\text { styrene } \\
\text { (ABS P400) }\end{array}$ & Rao (2016) \\
\hline $\begin{array}{l}\text { Artificial Neural } \\
\text { Network }\end{array}$ & $\begin{array}{ll}\text { - } & \text { Laser Power } \\
\text { - } & \text { Scanning Speed } \\
\text { - } & \text { Powder Feeding } \\
& \text { Rate } \\
\end{array}$ & $\begin{array}{ll}\text { - } & \text { Width } \\
\text { - } & \text { Depth } \\
\text { - } & \text { Height } \\
\end{array}$ & 2024 Al Alloy & $\begin{array}{l}\text { Caiazzo et. al } \\
\text { (2018) }\end{array}$ \\
\hline $\begin{array}{l}\text { Degradation and } \\
\text { Remaining } \\
\text { Useful Life } \\
\text { Prediction of } \\
\text { Aircraft Engines } \\
\text { Using Ensemble } \\
\text { Learning } \\
\end{array}$ & $\begin{array}{l}\text { RFs algorithm } \\
\text { is used to select } \\
\text { the most } \\
\text { important } \\
\text { variables }\end{array}$ & 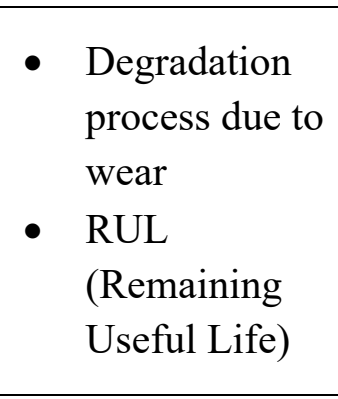 & $\begin{array}{l}\text { High Pressure } \\
\text { Compressor } \\
\text { and Fan } \\
\text { Module } \\
\text { (material is not } \\
\text { particularly } \\
\text { mentioned) }\end{array}$ & Li et. al (2019) \\
\hline $\begin{array}{l}\text { Experimentation } \\
\text { on Effects of } \\
\text { Melt Pool } \\
\text { Variables }\end{array}$ & Powder Flow Rate & $\begin{array}{ll}\text { - } & \text { Mean Surface } \\
& \text { Distribution } \\
\text { - } & \text { Mean Surface } \\
& \text { Roughness } \\
\text { - } & \text { Length of melt } \\
\text { pool } \\
\text { - } & \text { Depth of melt } \\
\text { pool } \\
\text { - } & \text { Area of melt } \\
& \text { pool }\end{array}$ & $\begin{array}{c}\text { Inconel } 718 \\
\text { powder on Ti- } \\
6 \mathrm{Al}-4 \mathrm{~V}\end{array}$ & Shah et. al (2010) \\
\hline
\end{tabular}




\begin{tabular}{|c|c|c|c|c|}
\hline $\begin{array}{c}\text { Supervised } \\
\text { learning using } \\
\text { KNearest } \\
\text { Neighbors } \\
\text { (KNN), Support } \\
\text { Vector Machine } \\
\text { (SVM), } \\
\text { Decision Tree } \\
\text { (DT) and } \\
\text { Discriminant } \\
\text { Analysis (DA). }\end{array}$ & $\begin{array}{l}\text { Features obtained via } \\
\text { functional principal } \\
\text { component analysis } \\
\text { (FPCA) }\end{array}$ & Porosity & Ti-6Al-4V & $\begin{array}{c}\text { Khanzadeh et. al } \\
\text { (2018) }\end{array}$ \\
\hline $\begin{array}{l}\text { XGBoost } \\
\text { Algorithm }\end{array}$ & $\begin{array}{l}\text { - Number of } \\
\text { Threads } \\
\text { - Number of } \\
\text { Training } \\
\text { Example } \\
\text { - Number of } \\
\text { Machines } \\
\end{array}$ & Time per Tree & Not Applicable & Chen et al (2016) \\
\hline $\begin{array}{c}\text { Sensitivity } \\
\text { analysis to open } \\
\text { black box data } \\
\text { mining models }\end{array}$ & $\begin{array}{l}\text { - Synthetic data } \\
\text { - Real-life data } \\
\text { on bank direct } \\
\text { marketing and } \\
\text { white wine } \\
\text { quality }\end{array}$ & Classification & Not applicable & $\begin{array}{c}\text { Cortez et. al } \\
\text { (2013) }\end{array}$ \\
\hline
\end{tabular}

\subsection{Research Gap}

Limitations of most of the existing data driven prediction methods lie in the high cost associate with the process, longer process time and elaborated size of data. On the other hand, outputs from the visual and simulation-based models can be far from the actual yields because they are often incapable of taking account the uncertainty that results from material variation or process parameters. Supervised models, on the contrary, are independent of design of experiment or the geometry of the specimens. This learning technique can be constructively used on the available data of any kind.

Though Finite Element Analysis (FEA) has been employed to model the mechanical behavior of printed parts, it is difficult to model the relationship between the parameters and mechanical behavior by using it. The advantage of FEA is that it can use cohesive elements to model bonded interfaces, but it is difficult to determine the boundary conditions at the bonded interface between 
the objects printed in DED process for FEA (Garg et. al, 2016). In the past decade Genetic Algorithms and Finite Element Analysis models have been popular choices to serve the purpose. Physics-based hybrid models combining Artificial Neural Network with Genetic Algorithm (Hayes et. al, 2017) with predictive capabilities were generated to predict mechanical properties of printed parts but these models faced difficulties to accomplish better prediction. Though lot of efforts have been made in building data driven predictive models to predict the mechanical behavior of printed parts of different AM processes, very few studies have been made on predictions of tensile behavior of DED fabricated parts.

\subsection{Challenges and Proposed Solution:}

Model selection is the most challenging part of this study. From the literature we found through the evaluation of predictive models, Neural Networks along with other machine learning algorithms like Ridge Regression, Random Forests, AdaBoost, Support Vector Regression, Lasso, Gradient Boosting performed very well and provided satisfactory results. To improve the prediction accuracy to the extreme extent, researchers is now drawn to develop and execute high performance machine learning methods which brings us to XGBoost (Extreme Gradient Boosting) method. It has turned into a state-of-art prediction method and a very popular choice of data scientists of this age. Speed and model performance enhancement are the chief concern while developing XGBoost. (Chen et. al, 2016). It supports three kinds of gradient boosting namely Gradient Boosting, Regularized Gradient Boosting, and Stochastic Gradient Boosting as the features of the scikit-learn. xGBoost is capable of supporting different range of computations with wide range of parameters with various size of the data set. While neural networks are proven to be capable of proving the best accuracy while predicting, they require a huge size of data set to function properly. On the other hand, XGBoost can provide satisfactory results with tabular or even comparatively smaller size of data. (Shwartz-Ziv et. al, 2021) According to the authors, on the same data set XGBoost outperformed deep learning algorithm 1D Convulational neural network, TabNet, DNF-Net and NODE.

XGBoost already has been used to perform data driven prediction modeling in a variety of additive manufacturing processes. The computational efficiency of XGBoost is compared to other machine learning algorithms is much higher (Zhang et. al, 2020). The authors observed that LSTM Neural Network runs 400 times slower than XGBoost. It performed extraordinarily well predicting tensile strength of the parts fabricated by cooperative 3D printing compared to SVR (Wu et. al, 2017). It is evident from the literature that this high-performance machine learning algorithm is yet to be used in prognosis of mechanical properties of Direct Energy Deposition process which inspired us to employ XGBoost to predict the effects of processing parameters on tensile behavior of parts fabricated by Laser based Direct Energy Deposition (L-DED).

The difficulties we faced during modeling are data preprocessing and Hyperparameter tuning. Special measures were taken in data preparation. The model was validated using the molten pool temperature data against time indices, layer indices, scanning speed, laser power and the height of the specimens. The hyperparameter, number of trees, shrinking rate, observation nodes, depth of trees are required to be tuned accordingly to get a better outcome. Design of experiment of the 
manufacturing phase was the most challenging part. To determine the number of layers for a particular recipe regarding the scanning speed and laser power to have a standard height of the specimen and to protect the deposition head in the deferential motion proved to be daunting as well. 


\section{Methodology}

This study includes two major phases- Experimentation Phase includes the design of the specimen, fabrication, strength testing and data collection and Predictive Modeling Phase in which the ML model is trained by the training data and then the prediction is compared with the test data to compare the accuracy of the prediction.

\subsection{Predictive Modeling:}

\subsubsection{Extreme Gradient Boosting (XGBoost)}

Extreme gradient boosting (XGBoost) is widely recognized machine learning algorithm and a very popular choice of data scientists. Developed by Chen et. al, 2016, this is an open source package. This ML algorithm is suitable for data mining challenges and also for tabular data type. XGBoost is faster than almost all other algorithms. The concept is descended from supervised learning technique with combination of Gradient boosting and Gradient Descent. Gradient descent is an iterative optimization algorithm suitable for function with several variables. The technique of gradient descent is to start with initial weights and over several iteration reach the local minima by updating the weights over and over. On the other hand, boosting involves sequential ensemble of learners. It consists of a cost function which finds the minimum value. It's desired to maintain a minimum gap between the predicted and real values and in order to do that the weights are required to help the cost function to reach its local minima. It's evident that, with error records, the weights are trained well and they can assist to reach the minimum value.

Gradient Boosting is a supervised learning following the principle of Gradient Descent and Boosting. Gradient Boosted Models (GBM's) are trees built sequentially. In GBM's, the weighted sum of multiple models is taken. It combines a set of weak learners and delivers improved prediction accuracy (Feng et. al, 2018). It is called gradient boosting because it uses a gradient descent algorithm to minimize the loss when adding new models. Decision trees are regarded as the weak learner in gradient boosting. Regression trees measure the gain from the loss function calculated from output for splits and sum up all the output following the similar procedure. Subsequent model's outputs are added in order to correct the residuals in the predictions which will be followed by the predecessor model. Each new model uses gradient descent optimization to update corrections to the weights.

The concept of boosting is to add new model to the ensemble. (Natekine et. al, 2013) After each iteration, a weak learner is added to the ensemble with the weights added from the error of the previous learner added to the model. From the mistakes/errors of the previous model, the weak learners are trained to improve the performance of the ensemble learner to be function as a single strong learner.

The purpose of building XGBoost is to aggregate all the computational advantages of boosting. XGBoost is an advanced implementation of GBM. GBM's build trees in series, but XGBoost built them parallel; making it faster than other ML algorithms. 
XGBoost dominates structured or tabular datasets on classification and regression predictive modeling problems. This approach supports both regression and classification predictive modeling problems. Gradient boosting involves three basic elements (Brownlee, 2018):

1. A loss function.

2. A weak learner

3. An additive model.

\subsubsection{XGBoost Mechanism}

Tree Building: The main concept of the tree building is to predict the residuals from the prediction and test data. At every iteration, a new tree is built and then the residuals are recomputed. This process keeps going until the model can fit the data well. A tree in XGBoost with a total of $J$ leaves. (Zhang et. al, 2020).

$$
T(x ; \Theta)=\sum_{j=1}^{J} \gamma_{j} I\left(x \in R_{j}\right)
$$

with parameters $\Theta=\left\{R_{j}, \gamma_{j}\right\}_{1}^{J}$ where $\gamma_{j}$ is the constant assigned to the disjoint region $R_{j}$. The boosted tree model can be expressed by the sum of all trees

$$
f_{M}(x)=\sum_{m=1}^{M} T\left(x ; \Theta_{m}\right)
$$

Loss Function: where $M$ represents the number of trees. In the forward stagewise procedure the objective function that needs to be optimized at each step is

$$
o b j_{m}=\sum_{i=1}^{n} L\left(y_{i}, f_{m-1}\left(x_{i}\right)+v \cdot T\left(x_{i} ; \Theta_{m}\right)\right)+\varepsilon \cdot J+\frac{1}{2} \lambda \cdot \sum_{j=1}^{J} \gamma_{j m}{ }^{2}
$$

where $\boldsymbol{v}$ is the shrinkage factor while $\boldsymbol{\varepsilon}$ and $\lambda$ are the regularization parameters to reduce the overfitting. The first part of the objective function, $\sum_{i=1}^{n} \boldsymbol{L}\left(\boldsymbol{y}_{\boldsymbol{i}}, \boldsymbol{f}_{\boldsymbol{m}-\mathbf{1}}\left(\boldsymbol{x}_{\boldsymbol{i}}\right)+\boldsymbol{v} \cdot \boldsymbol{T}\left(\boldsymbol{x} ; \boldsymbol{\Theta}_{\boldsymbol{m}}\right)\right)$ measures the loss for using $\boldsymbol{f}_{\boldsymbol{m}}\left(\boldsymbol{x}_{\boldsymbol{i}}\right)$ to predict $y_{i}$. The prediction $\boldsymbol{f}_{\boldsymbol{m}}\left(\boldsymbol{x}_{\boldsymbol{i}}\right)$ in XGBoost is combined by the prediction from previous trees $\boldsymbol{f}_{\boldsymbol{m - 1}}\left(\boldsymbol{x}_{\boldsymbol{i}}\right)$ and the scaled approximation from the $m$-th tree $\boldsymbol{v}$. $\boldsymbol{T}\left(\boldsymbol{x} ; \boldsymbol{\Theta}_{\boldsymbol{m}}\right)$. The second part of the objective function, $\boldsymbol{\varepsilon} \cdot \boldsymbol{J}+\frac{\mathbf{1}}{\mathbf{2}} \lambda \cdot \sum_{j=1}^{J} \gamma_{j \boldsymbol{m}}{ }^{2}$ defines the complexity of the tree $\boldsymbol{T}\left(\boldsymbol{x} ; \boldsymbol{\Theta}_{\boldsymbol{m}}\right)$.

Split: XGBoost trees use binary splitting. And the split point selection starts with searching the point where the gain is maximum. During the tree pruning process, a gain function is calculated with

$$
\text { Gain }=\frac{1}{2}\left[\frac{G_{L}^{2}}{H_{L}+\lambda}+\frac{G_{R}^{2}}{H_{R}+\lambda}-\frac{\left(G_{L}+G_{R}\right)^{2}}{H_{L}+H_{R}+\lambda}\right]-\gamma
$$

where $G_{L}, H_{L}, G_{R}$ and $H_{R}$ represent the sum of $g_{i}$ and $h_{i}$ at the left and right branch after splitting, respectively. $g_{i}$ and $h_{i}$ are the first and second derivatives of $\boldsymbol{L}\left(\boldsymbol{y}_{\boldsymbol{i}}, \boldsymbol{f}_{\boldsymbol{m}-\mathbf{1}}\left(\boldsymbol{x}_{\boldsymbol{i}}\right)\right)$. To make the 
optimal split at each node, the branch with the maximum value of gain is selected. $\boldsymbol{\gamma}$ is a constant assigned as a tuning factor. The unique features of using second order Taylor expansions in the loss function and emphasizing the regularization terms in the objective function make XGBoost powerful and efficient in handling different modeling tasks. (Zhang et. al, 2020)

Pruning: XGBoost performs an optional function called Pruning after the tree models are built. Pruning starts from the bottom with the leaves and then moves up to the root node. The purpose of this movement is to check if the gain function at each node is smaller or larger than the $\gamma$ value. If the gain value is smaller than the $\gamma$ value, then the pruner cuts the node and reach for the next one. If the gain value is higher than the $\gamma$ value, the pruner leaves the whole parent node without checking any further.

\subsubsection{Model Architecture:}

The overall parameters have been divided into 3 categories by XGBoost creators. (Maladkar, 2018) They are:

1. General Parameters: Guide the overall functioning

2. Booster Parameters: Guide the individual booster (tree/regression) at each step

3. Learning Task Parameters: Guide the optimization performed

Regularization: This belongs mainly to the learning task parameters. Regularization is a weight control technique which is required to control the overfitting of the data in a model. The regularization parameters exist in XGBoost are:

- $\quad$ L2 regularization, $\lambda$ : This is a constant added to the second part of the loss function. It helps to control the size of the weights and selection of the spilt point.

- L1 regularization, $\boldsymbol{\alpha}$ : Similar to L2 regularization, function of L1 function is also to act on the weight control of the model.

- Learning rate, $\boldsymbol{\varepsilon}$ : This is also a constant less than 1 which is multiplied to the number of leaves $\boldsymbol{J}$ to slow down the speed of the model to fit a data and controls overfitting.

Pruning: As mentioned before pruning occurs as an optional function of XGBoost. It belongs to the booster parameters class.

Gamma $\gamma$ : A fixed constant as a threshold value to be used in improvement of the gain function. It helps to decide the pruning. And also split points depend on the $\boldsymbol{\gamma}$ value.

min_child_weight: This is the minimum value of the second derivative at a child node to be able to participate in the splitting.

max_depth: This is actually the maximum depth a tree can grow. This plays as a hard stop to halt the tree building process.

Early stopping: Early stopping is not set during the model initialization rather it is set during the training. This helps the model to select number of estimators during the hyperparameter tuning. 
Hyperparameters: Hyperparameters are not related to data rather they are the set of configurations which assist the model to fit the certain kind of data or problem. Typically, a hyperparameters has predictable effects on the model but still it's the most challenging part during building a model. Therefore, we need to search for the best set of hyperparameters for the model to be able to predict better. This is called hyperparameter optimization, hyperparameter tuning, or hyperparameter search. Hyperparameters are not the model parameters and it is not possible to find the best set just from the training data. (Maladkar, 2018)

A range of different optimization algorithms can be used. The two most popular techniques are Grid Search and Random Search.

Grid Search: Grid search is a technique which is designed to find the right set of the hyperparameters for the data set in a manner that it considers and employ every set of values in the grid one by one. Each set of parameters is taken into consideration and the accuracy is noted. Once all the combinations are evaluated, the model with the set of parameters which give the top accuracy is considered to be the best. The cons of this technique are it takes an incredible amount of time to compute the best result and it use up a lot of spaces of the core. (Maladkar, 2018)

Random Search: Random search is a technique which select the hyperparameters randomly form the set. This methodology chose random combinations of the hyperparameters are used to find the best solution for the built model. It is similar to grid search, but it is able to determine the optimized set of the hyperparameters better than grid search. The limitation of random search is that it yields high variance during performing due to random selection of parameters compare to the grid search. (Maladkar, 2018)

The hyperparameter set is selected based on the available literatures and experimentations bellow:

Table 2: Summary of Architecture of the model

\begin{tabular}{|c|c|}
\hline Language & Python 3.7 \\
\hline Environment & Conda \\
\hline Library & Scikit Learn \\
\hline Model & XGRegressor \\
\hline Hyperparameters Tuning & RandomizedSearchcv \\
\hline n_estimators & $500,1000,2000$ \\
\hline Max_depth & $4,5,6$ \\
\hline Min_child_weight & $1,2,3$, \\
\hline Learning Rate & $0.1,0.01$ \\
\hline n_iter & $2,3,6$ \\
\hline
\end{tabular}


The flow chart of how XGBoost works from beginning to performance evaluation.

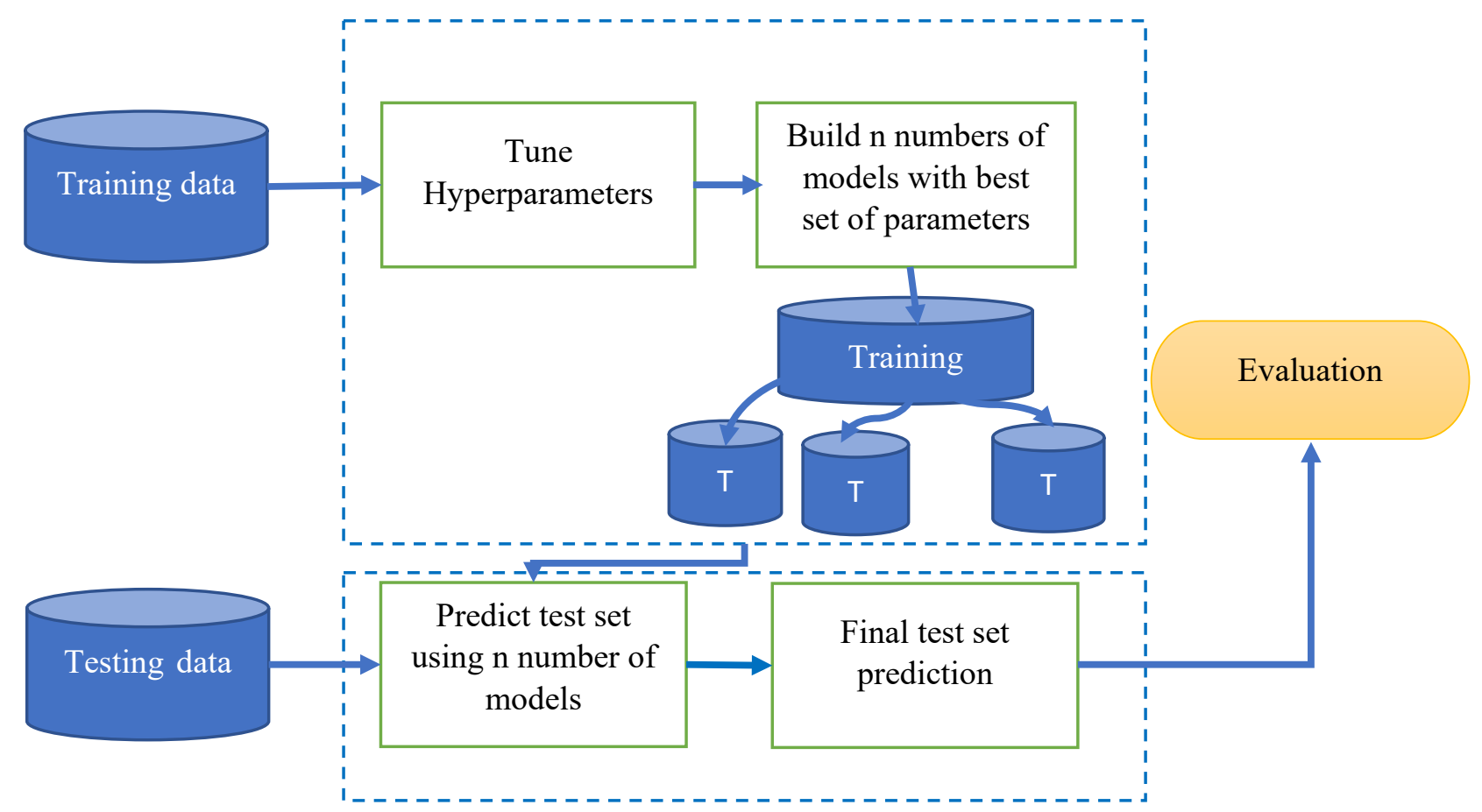

Figure 1: Flowchart of XGBoost

The modeling can be divided into two main phases. First the training phase where the data is loaded to model. Then the chief concern is to tune the hyperparameters according to the data and the problem type. The $\mathrm{n}$ number of trees or models are built where the objective values being minimized, the residuals are computed consequent and weight is given to the errors of the previous models for the new model to learn. This is how the training phase is completed. Then at the beginning of the testing phase, the test data is either selected by the user or model picks them using different split functions. The learning experience is applied to the test data to fit the best set of the results. When the final prediction is completed, the prediction evaluation is calculated to check the performance accuracy of the model. In our work, first in case of melt pool data, we have selected the test data but later for the tensile testing, the split function is used to separate test data from the training data.

\subsubsection{Model Validation}

\subsubsection{Data Preprocessing}

For validating the model, we have used the data provided by Dr. Liu from Zhang et. al, 2020, a collaboration work of Dr. Liu with Ziyang Zhang. Data preprocessing is one of the most critical part of the modeling. The experimental setup in Zhang et. al, 2020 is thin walled Inconel 718 specimens with eight layers were fabricated under different experimental conditions. A total of twenty-five sets of molten pool temperature data were collected with a full experiment design. Each data set records the molten pool temperature of eight layers and for data preprocessing, the measured temperature data during dwell time was removed for all 25 experiments. 
Table 3: Design of experiment of molten pool temperature

\begin{tabular}{|c|c|c|c|c|c|c|}
\hline $\begin{array}{c}\text { Experiments } \\
\text { No. }\end{array}$ & $\begin{array}{c}\text { Laser } \\
\text { power } \\
\text { (W) }\end{array}$ & $\begin{array}{l}\text { Scanning } \\
\text { speed } \\
(\mathbf{m m} / \mathbf{s})\end{array}$ & \multicolumn{3}{|c|}{$\begin{array}{c}\text { Height } \\
(\mathbf{m m})\end{array}$} & $\begin{array}{c}\text { Ave. } \\
\text { Height } \\
\text { (mm) }\end{array}$ \\
\hline 1 & 250 & 11 & 1.54 & 1.53 & 1.51 & 1.53 \\
\hline 2 & 275 & 11 & 1.57 & 1.34 & 1.44 & 1.45 \\
\hline 3 & 300 & 11 & 1.83 & 1.90 & 1.76 & 1.83 \\
\hline 4 & 250 & 9 & 1.95 & 1.96 & 2.07 & 1.99 \\
\hline 5 & 325 & 11 & 1.74 & 1.66 & 1.59 & 1.66 \\
\hline 6 & 275 & 9 & 1.64 & 1.79 & 1.89 & 1.77 \\
\hline 7 & 350 & 11 & 1.71 & 1.81 & 1.80 & 1.77 \\
\hline 8 & 300 & 9 & 2.23 & 2.24 & 2.33 & 2.27 \\
\hline 9 & 250 & 7 & 3.01 & 3.06 & 3.03 & 3.03 \\
\hline 10 & 325 & 9 & 2.37 & 2.40 & 2.45 & 2.41 \\
\hline 11 & 350 & 9 & 2.48 & 2.61 & 2.32 & 2.47 \\
\hline 12 & 275 & 7 & 2.73 & 2.84 & 2.80 & 2.79 \\
\hline 13 & 300 & 7 & 3.07 & 3.06 & 3.14 & 3.09 \\
\hline 14 & 325 & 7 & 3.22 & 3.27 & 3.29 & 3.26 \\
\hline 15 & 250 & 5 & 4.37 & 4.70 & 4.52 & 4.53 \\
\hline 16 & 350 & 7 & 2.56 & 2.58 & 2.52 & 2.55 \\
\hline 17 & 275 & 5 & 4.38 & 4.39 & 4.73 & 4.50 \\
\hline 18 & 300 & 5 & 4.37 & 4.08 & 3.98 & 4.14 \\
\hline 19 & 325 & 5 & 3.95 & 3.94 & 3.78 & 3.89 \\
\hline 20 & 350 & 5 & 3.48 & 3.47 & 3.60 & 3.52 \\
\hline 21 & 250 & 3 & 5.42 & 5.48 & 5.58 & 5.49 \\
\hline 22 & 275 & 3 & 5.16 & 5.25 & 5.39 & 5.27 \\
\hline 23 & 300 & 3 & 4.83 & 5.01 & 5.22 & 5.02 \\
\hline 24 & 325 & 3 & 5.14 & 5.25 & 5.21 & 5.20 \\
\hline 25 & 350 & 3 & 5.03 & 4.86 & 4.88 & 4.92 \\
\hline
\end{tabular}




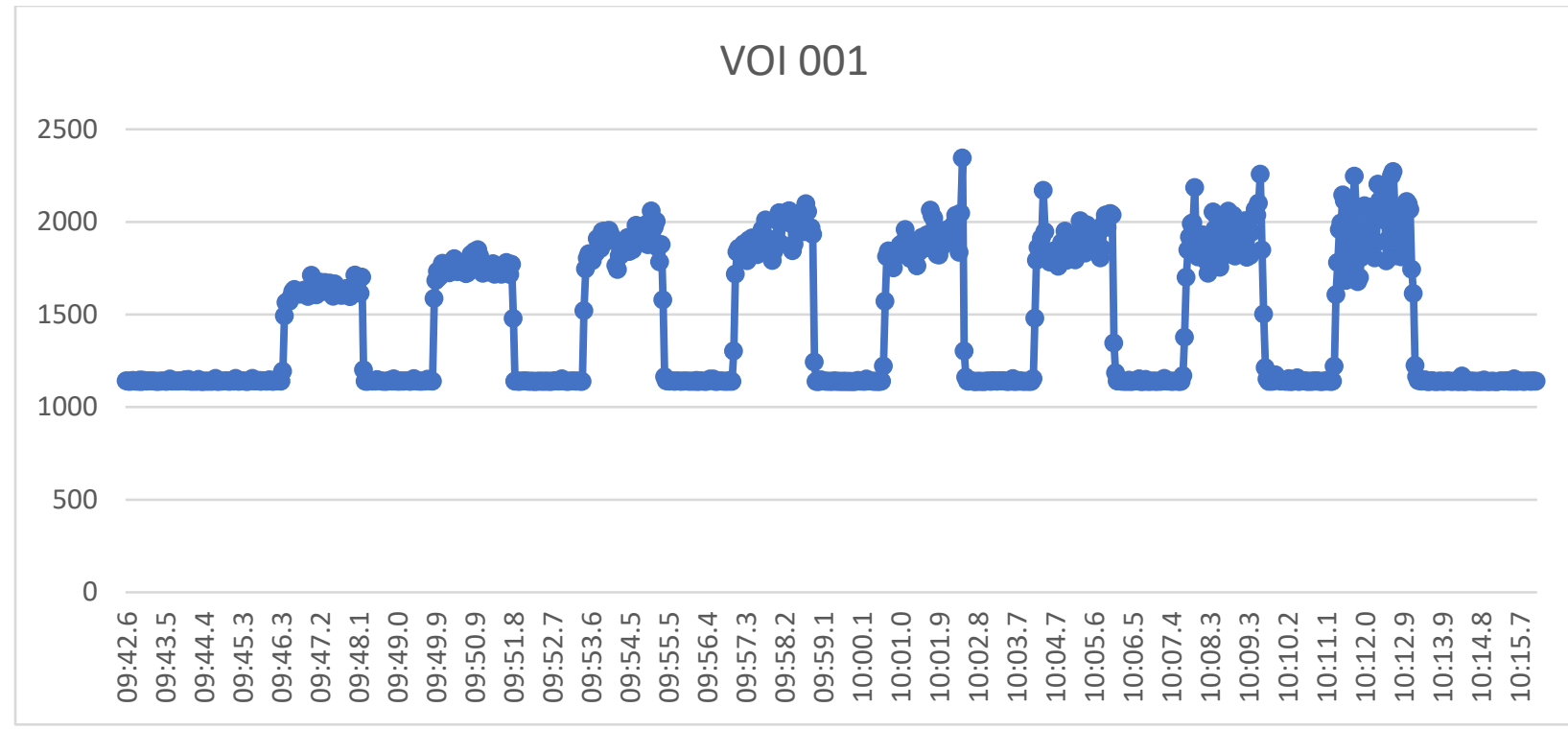

Figure 2: The molten pool temperature of Experiment 1 (laser power: $250 \mathrm{~W}$, scanning speed:11 $\mathrm{mm} / \mathrm{s}$ )

Dwell temperature data is removed from the data set as noises in Figure 3. They are function of only time indexing. During dwelling the laser is off, so there is no heating. The dwell temperature ranged from very lower than the molten pool temperature. The final data of the experiment 1 is below:

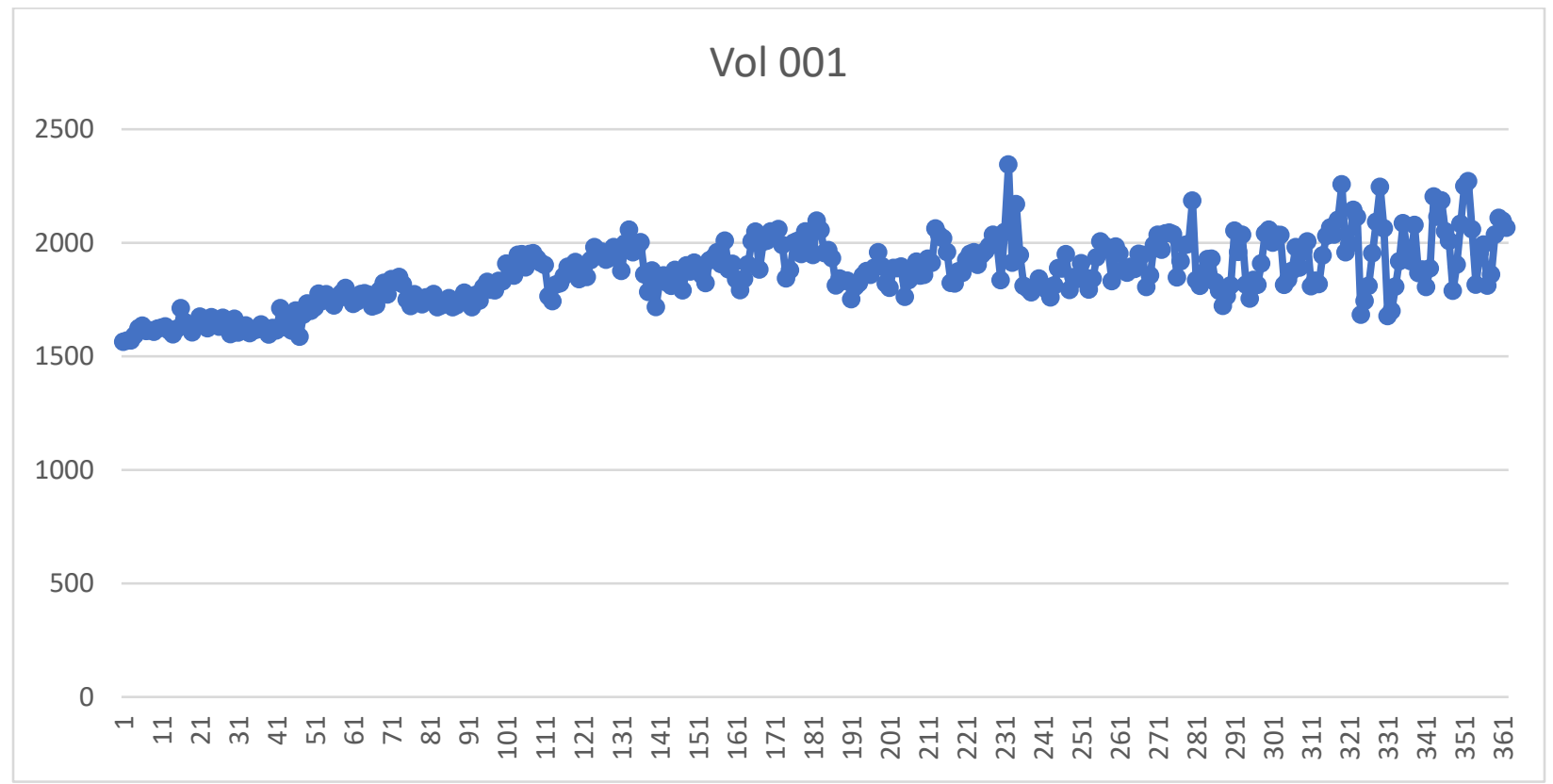

Figure 3: Molten pool temperature of eight layers of the specimen of experiment 1 after removing the dwelling temperature data. 
Each layer contains on average 50 to 100 data of time indexing. Layer indexing is also included for every temperature point. The three most affecting parameters Laser Power, Scanning Speed and Specimen Heights (Zhang et al., 2020) which have direct impact on the molten pool temperature are assigned along with a particular experiment. Four cases are constructed where in each one, only one random experiment data is assigned for testing and rest twenty-four served for the training.

Table 4. Training and test data sizes for different cases

\begin{tabular}{|l|l|l|l|l|l|l|}
\hline Cases & $\begin{array}{l}\text { Training } \\
\text { data }\end{array}$ & Test data & $\begin{array}{l}\text { Laser power } \\
\text { of test data }\end{array}$ & $\begin{array}{l}\text { Scanning } \\
\text { speed of test } \\
\text { data }\end{array}$ & $\begin{array}{l}\text { Training } \\
\text { data size }\end{array}$ & $\begin{array}{l}\text { Test } \\
\text { data } \\
\text { size }\end{array}$ \\
\hline Case 1 & $\# 2-25$ & $\# 1$ & $250 \mathrm{~W}$ & $11 \mathrm{~mm} / \mathrm{s}$ & 17167 & 361 \\
\hline Case 2 & $\# 1-6,8-25$ & $\# 7$ & $350 \mathrm{~W}$ & $11 \mathrm{~mm} / \mathrm{s}$ & 17162 & 367 \\
\hline Case 3 & $\# 1-20,22-$ & $\# 21$ & $250 \mathrm{~W}$ & $3 \mathrm{~mm} / \mathrm{s}$ & 16200 & 1328 \\
\hline Case 4 & $\# 1-24$ & $\# 25$ & $350 \mathrm{~W}$ & $3 \mathrm{~mm} / \mathrm{s}$ & 16199 & 1329 \\
\hline
\end{tabular}

\subsubsection{Results of the model:}

The four cases were plotted to demonstrate the accuracy of the prediction of the model. The prediction evaluation was done by measuring the Root mean square error (RMSE) value.

$$
R M S E=\sqrt{\sum_{i=1}^{n} \frac{(\widehat{Y l}-Y i)}{n}}
$$

$Y_{i}$ and $\widehat{Y}_{l}$ denote the observed value and predicted value respectively. The RMSE is smaller when the prediction is closer to real values and higher values mean the model failed to predict closely. Compared to the very recent work of Zhang et al., 2020 on the very same data set with XGBoost, the results are shown into the table.

Table 5 Comparison of RMSE's

\begin{tabular}{|c|c|c|c|c|}
\hline \#Cases & Training Data & Testing data & RMSE & $\begin{array}{c}\text { RMSE (Zhang } \\
\text { et. al, 2020) }\end{array}$ \\
\hline Case 1 & $\# 2-25$ & $\# 1$ & 94 & 96 \\
\hline Case 2 & $\# 1-6,8-25$ & $\# 7$ & 35 & 25 \\
\hline Case 3 & $\# 1-20,22-25$ & $\# 21$ & 43 & 43 \\
\hline Case 4 & $\# 1-24$ & $\# 25$ & 30 & 24 \\
\hline
\end{tabular}

The reasons of deviation from the results of Zhang et al. 2020, the data set was prepared separately. Removal of the dwelling temperatures might differ in both data sets. And the hyperparameter tuning was different in both works. 


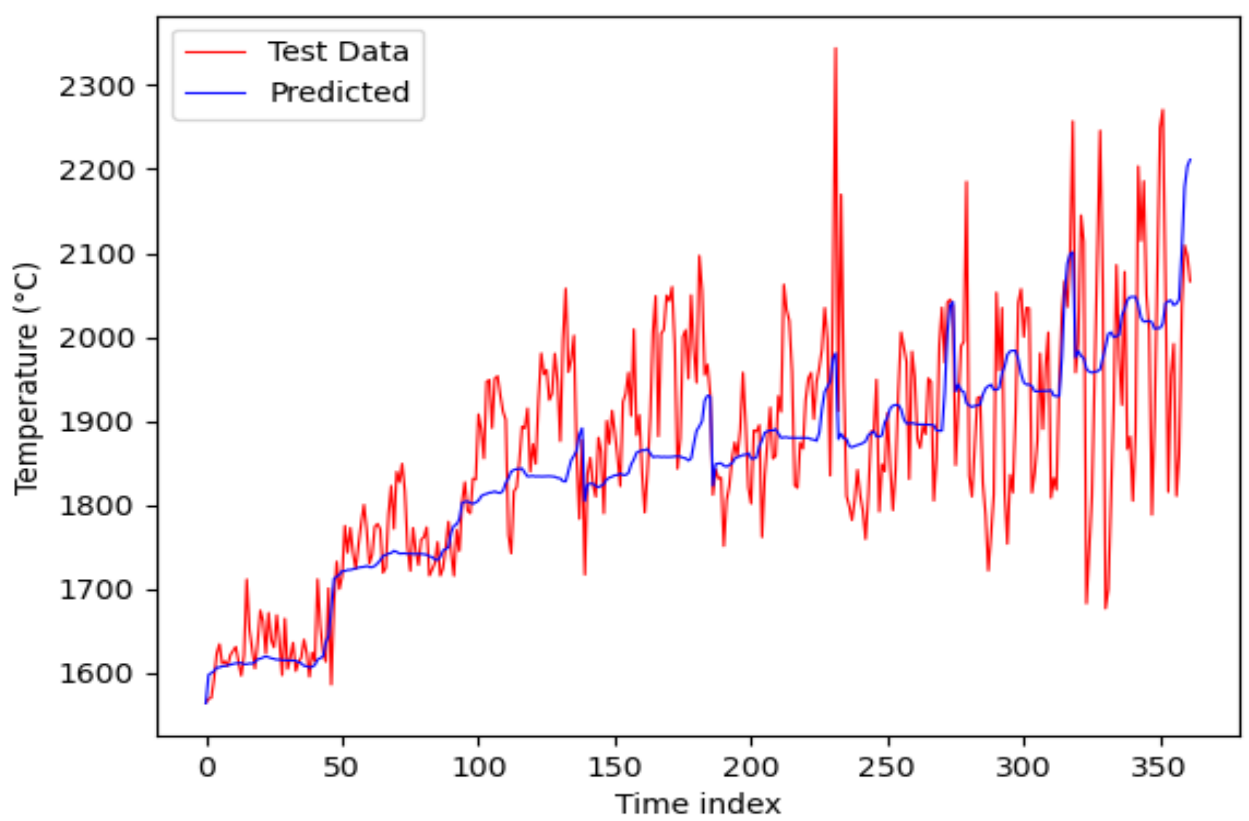

Figure 4: Predicted and observed molten pool temperature of experiment 1 (laser power: $250 \mathrm{~W}$, scanning speed: $11 \mathrm{~mm} / \mathrm{s}$ )

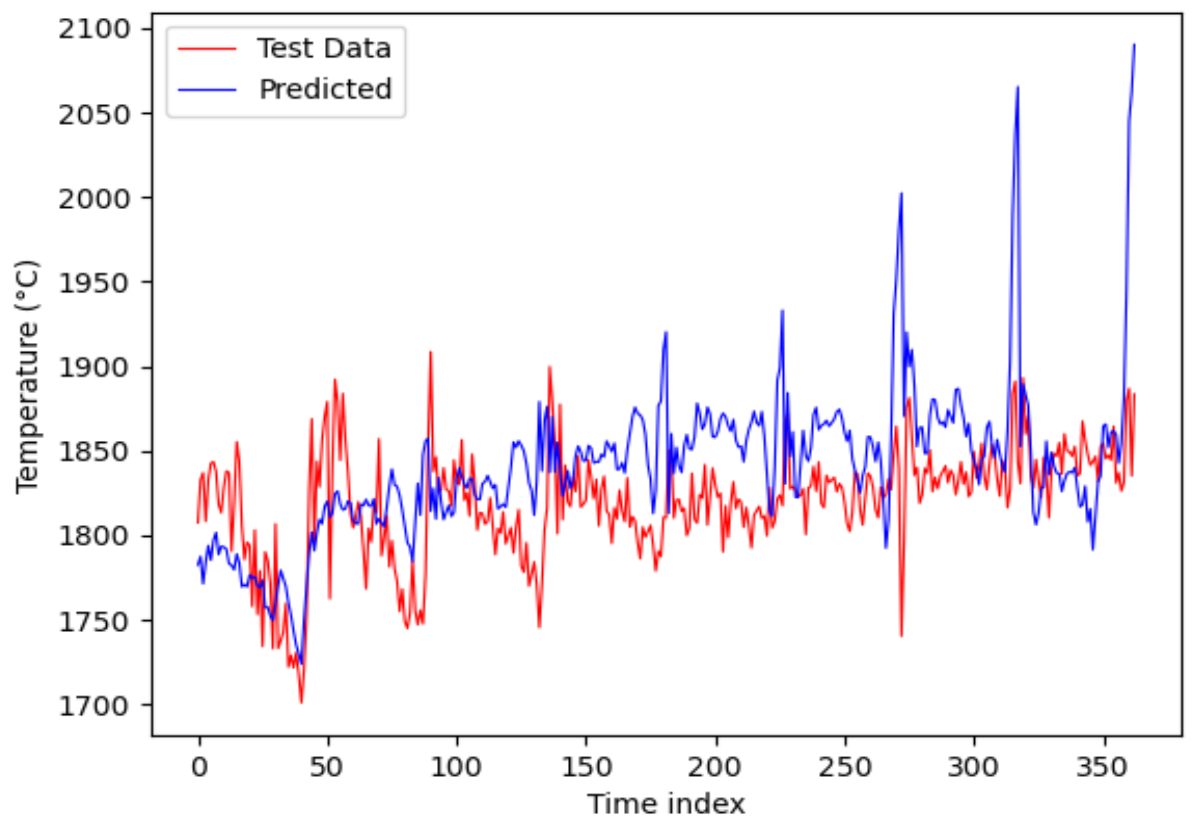

Figure 5: Predicted and observed molten pool temperature of experiment 7 (laser power: $350 \mathrm{~W}$, scanning speed: $7 \mathrm{~mm} / \mathrm{s}$ ) 


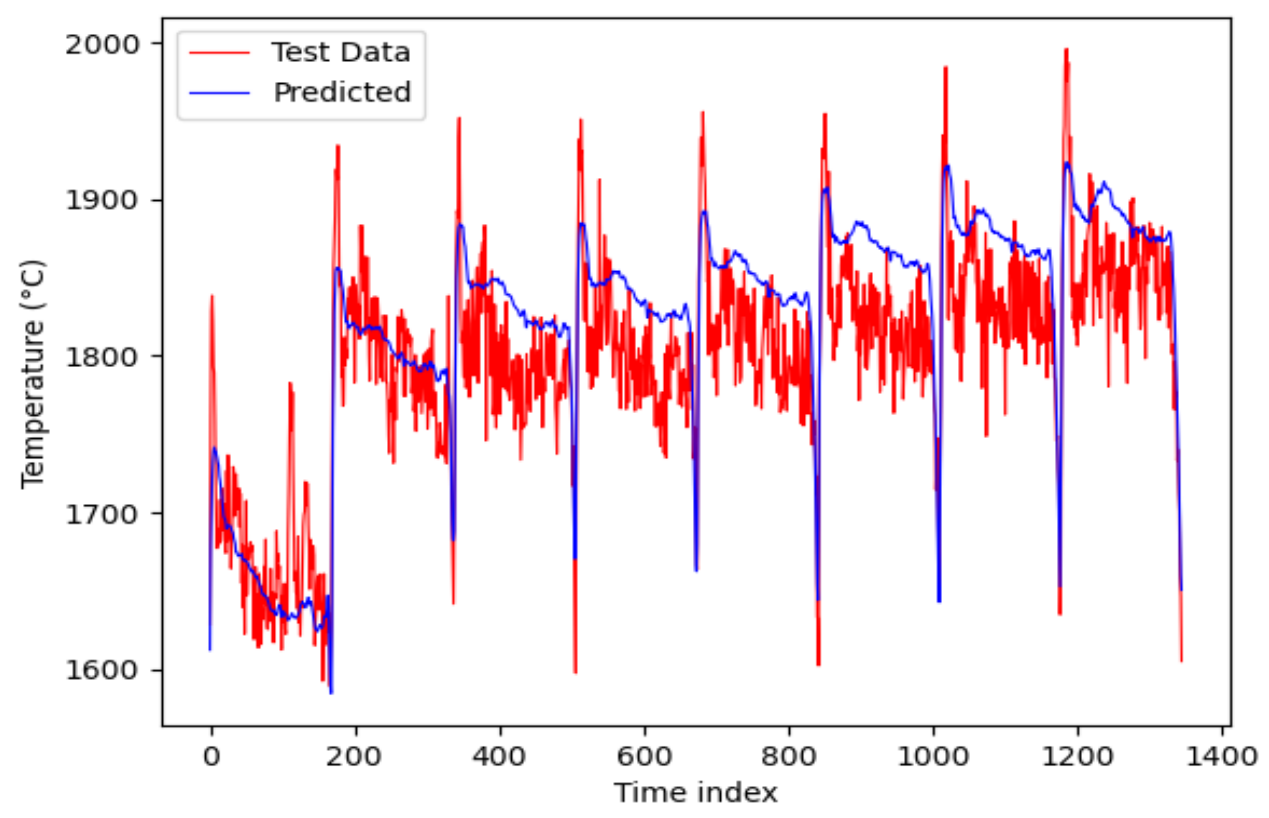

Figure 6: Predicted and observed molten pool temperature of experiment 21 (laser power: 250 $W$, scanning speed: $3 \mathrm{~mm} / \mathrm{s}$ )

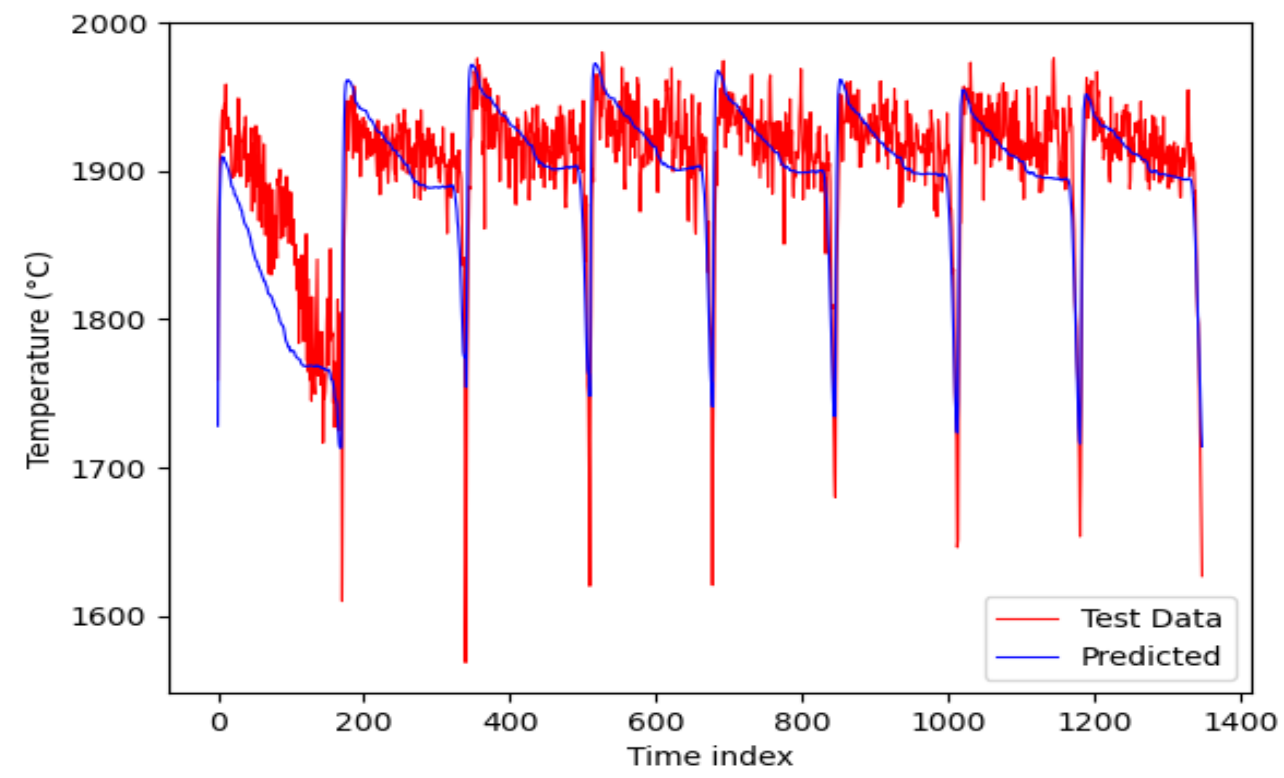

Figure 7: Predicted and observed molten pool temperature of experiment 25 (laser power: 350 $W$, scanning speed: $3 \mathrm{~mm} / \mathrm{s}$ ) 


\subsection{Data Collection}

\subsubsection{Material Selection}

316 Stainless Steel (UNS S31600) are molybdenum bearing austenitic stainless steel which is better wear resistant than other stainless steel. This alloy is better with high creep, higher tensile strength properties and widely used for durable and high stress situations. (Montemor et. al, 1999)

Table 6: Chemical composition of 316 stainless steel

\begin{tabular}{cccccccccc}
\hline $\mathrm{C}$ & $\mathrm{Mn}$ & $\mathrm{Si}$ & $\mathrm{Cr}$ & $\mathrm{Ni}$ & $\mathrm{Mb}$ & $\mathrm{P}$ & $\mathrm{S}$ & $\mathrm{N}$ & $\mathrm{Fe}$ \\
\hline 0.08 & 2 & 0.75 & 16 & 10 & 2 & 0.045 & 0.03 & 0.1 & Bal. \\
\hline
\end{tabular}

\subsubsection{Specimen Design}

Dog bone specimens are a common design for tensile strength test. According to the ASTM standard, the total length of the dog bone specimen should be $165 \mathrm{~mm}$, width $19 \mathrm{~mm}$ and the thickness $3.2 \mathrm{~mm}$. (ASTM52900-15) In our work, the standard dimensions are scaled down to half: length $82.5 \mathrm{~mm}$, width $9.50 \mathrm{~mm}$ but keeping the height $3 \mathrm{~mm}$ to avoid laborious printing time and powder waste. There are 5 layers and height of each layer is $0.54 \mathrm{~mm}$. Though 316 Stainless Steel is selected for the powder material, low carbon steel is used as substrate.

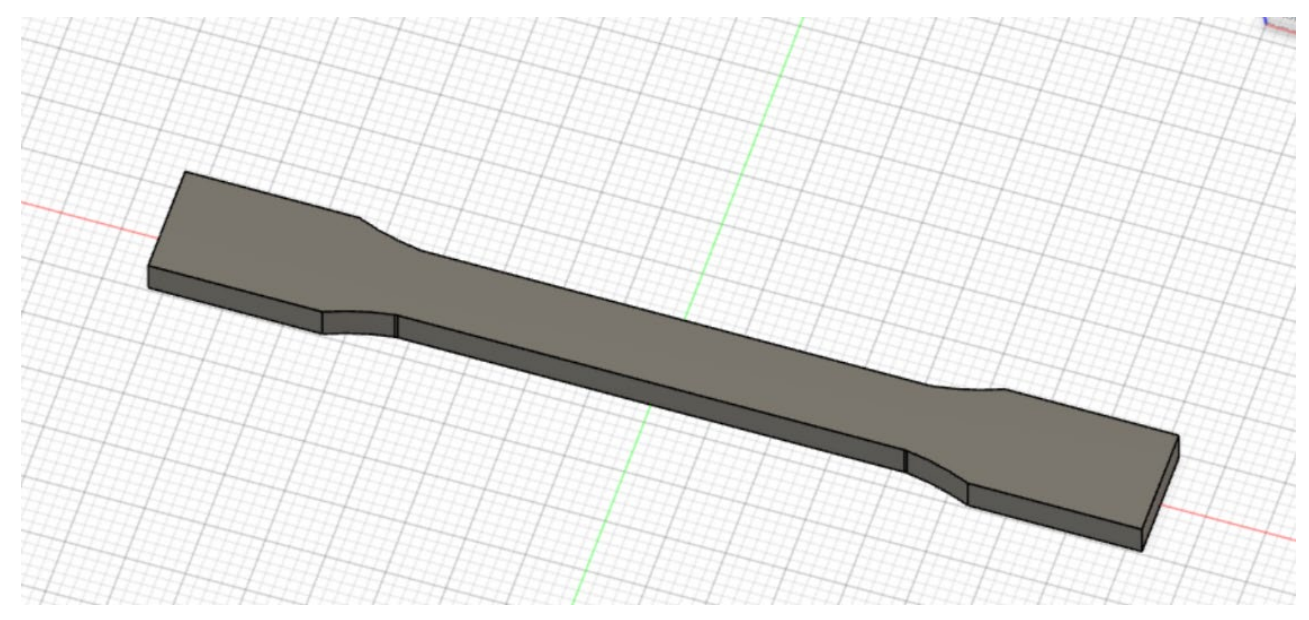

Figure 8: Final design of Dog bone specimen

\subsubsection{Design of Experiment:}

Total 25 dog bone specimens are printed on low carbon steel, plain and rectangular substrate using 25 different recipes. There are 5 groups based on 5 different scanning speeds. The design of experiment is shown in the table below. Powder feed rate, laser spot diameter, nozzle gas, shield gas and carrier gas supply rate were constant for all the experiments. From these 25 Dog bones with 5 layers, each sample will be cut from the original one so that we finally can have each specimen per recipe. Total cycle time per specimen is on average 13 minutes. 
Table 7: Design of experiment.

\begin{tabular}{|c|c|}
\hline Process parameters & Values \\
\hline Laser Power $(\mathrm{W})$ & $600,700,800,900,1000$ \\
\hline Scanning Speed $(\mathrm{mm} / \mathrm{s})$ & $8,9,10,11,12$ \\
\hline Powder Feed Rate $(\mathrm{g} / \mathrm{mm})$ & 5 \\
\hline Laser Spot Diameter, $(\mathrm{mm})$ & 2 \\
\hline Nozzle Gas $(\mathrm{L} / \mathrm{min})$ & 9 \\
\hline Shield Gas $(\mathrm{L} / \mathrm{mm})$ & 12 \\
\hline Carrier Gas $(\mathrm{L} . \mathrm{mm})$ & 5 \\
\hline Hatch Space $(\mathrm{mm})$ & 0.54 \\
\hline
\end{tabular}

Table 8: Design of Experiment with groups of parameters

\begin{tabular}{|c|c|c|c|c|}
\hline $\begin{array}{c}\text { Group } \\
\text { No }\end{array}$ & $\begin{array}{c}\text { Laser Power } \\
(\mathbf{W})\end{array}$ & $\begin{array}{c}\text { Scanning Speed } \\
(\mathbf{m m} / \mathbf{s})\end{array}$ & $\begin{array}{c}\text { Layer Height } \\
(\mathbf{m m})\end{array}$ & $\begin{array}{c}\text { Energy Density } \\
\left(\mathbf{J} / \mathbf{m m}^{\mathbf{2}}\right)\end{array}$ \\
\hline 1 & 600 & 8 & 1.51 & 18.8 \\
\hline 1 & 700 & 8 & 1.52 & 21.9 \\
\hline 1 & 800 & 8 & 1.75 & 25.0 \\
\hline 1 & 900 & 8 & 1.8 & 28.1 \\
\hline 1 & 1000 & 8 & 1.75 & 31.3 \\
\hline 2 & 600 & 9 & 1.32 & 16.7 \\
\hline 2 & 700 & 9 & 1.47 & 19.4 \\
\hline 2 & 800 & 9 & 1.34 & 22.2 \\
\hline 2 & 900 & 9 & 1.37 & 25.0 \\
\hline 2 & 1000 & 9 & 1.35 & 27.8 \\
\hline 3 & 600 & 10 & 0.79 & 15.0 \\
\hline 3 & 700 & 10 & 1.71 & 17.5 \\
\hline 3 & 800 & 10 & 1.7 & 20.0 \\
\hline 3 & 900 & 10 & 1.77 & 22.5 \\
\hline 3 & 1000 & 10 & 1.83 & 25.0 \\
\hline 4 & 600 & 11 & 1.38 & 13.6 \\
\hline 4 & 700 & 11 & 1.41 & 15.9 \\
\hline 4 & 800 & 11 & 1.49 & 20.5 \\
\hline 4 & 900 & 11 & 1.62 & 22.7 \\
\hline 4 & 1000 & 11 & 1.73 & 12.5 \\
\hline 5 & 600 & 12 & 1.27 & 14.6 \\
\hline 5 & 700 & 12 & 1.42 & 16.7 \\
\hline 5 & 800 & 12 & 1.51 & 20.8 \\
\hline 5 & 900 & 12 & 1.54 & \\
\hline 5 & 1000 & 12 & 1.37 & \\
\hline
\end{tabular}




\subsubsection{Experiment Setup}

Materials tensile behaviors, such as ultimate tensile strength, elongation, yield strength, is collected from the tensile test experiments. Dog bone specimen preparation and testing consisted of three stages-

1. Dog bone specimen fabrication with DED

2. EDM of the specimen

3. Tensile test

\subsubsection{Dog bone specimen fabrication with DED:}

The most common type of DED system is a powder-based laser deposition system optimized for metals. In Laser based DED, a deposition head is utilized to deposit material onto the substrate. A deposition head consists of laser optics, powder nozzles, inert gas tubing. The substrate can be either a flat one on which a new part will be fabricated or an existing part on which additional geometry can be added. There is a relative differential motion between the substrate and deposition head to control the deposition.

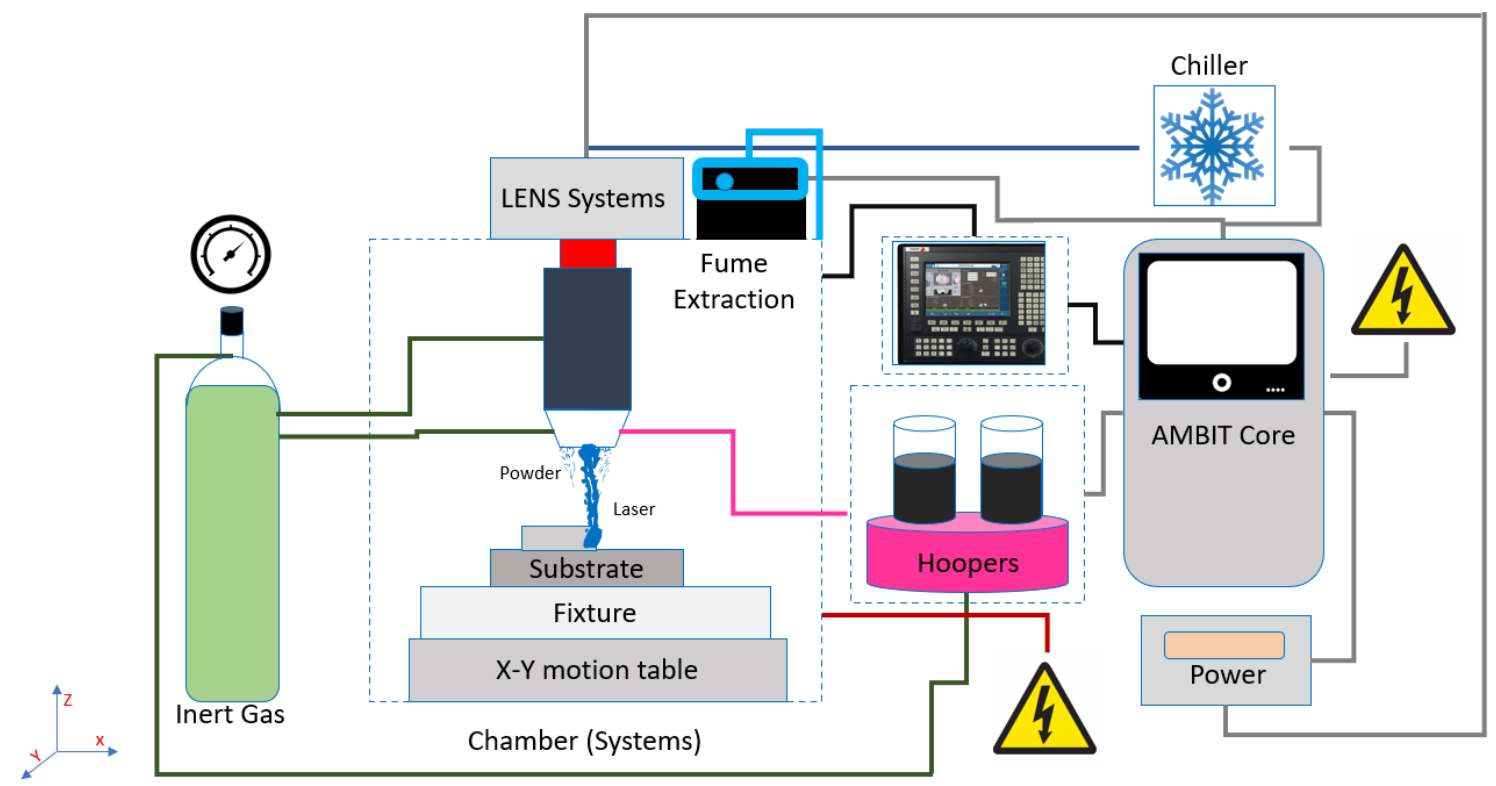

Figure 9: Schematic diagram of AMBIT ${ }^{T M}$ laser based Directed Energy Deposition Hybrid System

The AMBIT ${ }^{\mathrm{TM}}$ core L-DED machine shown in the figure is consisted of an inert gas supply, a system chamber including the X-Y axes motion table, the deposition head and the lens system, powder hoppers, AMBIT core, operator control, a chiller and a fume extractor. Inert gas is supplied to the system's chamber to the deposition head, AMBIT core controls the laser power, powder 
delivery, cooling down cycle with the fume extraction and operator control. Using operator control the deferential motion between the motion table and the deposition head is monitored.

The G code is generated by Autodesk Fusion 360. In DED, a subsequent layer is typically deposited in a different orientation than the previous layer. Common scan patterns from layer to layer are usually multiples of $30^{\circ}, 45^{\circ}$, and $90^{\circ}$. (Gibson et. al, 2015)

Layer orientations can also be randomized between layers. In our setup, the first and fifth layers are at $0^{\circ}$, second layer at $45^{\circ}$, third layer at $135^{\circ}$, fourth layer at $45^{\circ}$. They are exhibited in table 9. The orientation variation from layer to layer eliminates preferential grain growth, which otherwise makes the properties anisotropic and reduces residual stresses. (Gibson et. al, 2015)

Table 9: Infill Structure of Each layer

\begin{tabular}{|c|c|c|c|c|c|}
\hline Layers \# & 1 & 2 & 3 & 4 & 5 \\
\hline $\begin{array}{c}\text { Infill } \\
\text { Structure }\end{array}$ & & & \\
\hline
\end{tabular}

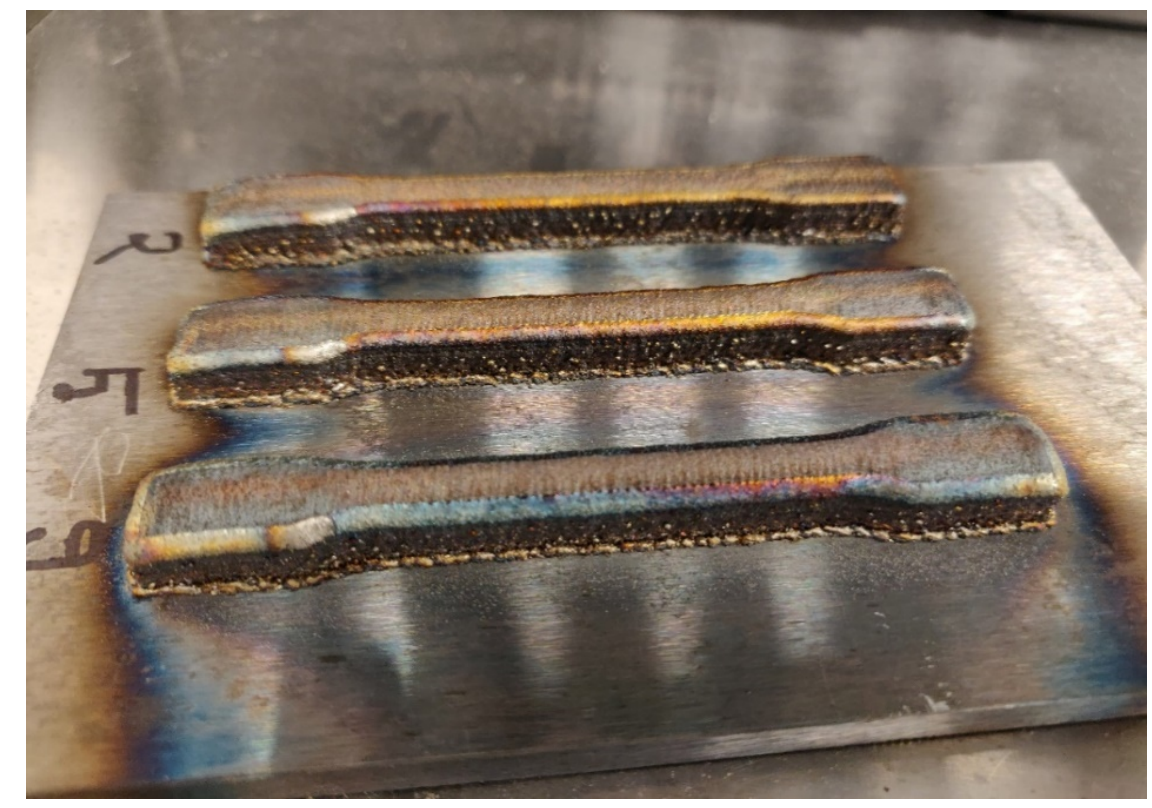

Figure 10: 3D printed 316 SS Dog bone specimens by L-DED process. 


\subsubsection{EDM of the dog bone parts:}

We have employed electric discharge machining for the subtraction of the dog bones from the base. Electric Discharge Machining (EDM) is a programmable metal removal process which uses electric spark as a cutting tool. This is an electro-thermal non-traditional machining where electric energy is used to create the spark and thermal energy for material removal (IIT, kanpur).
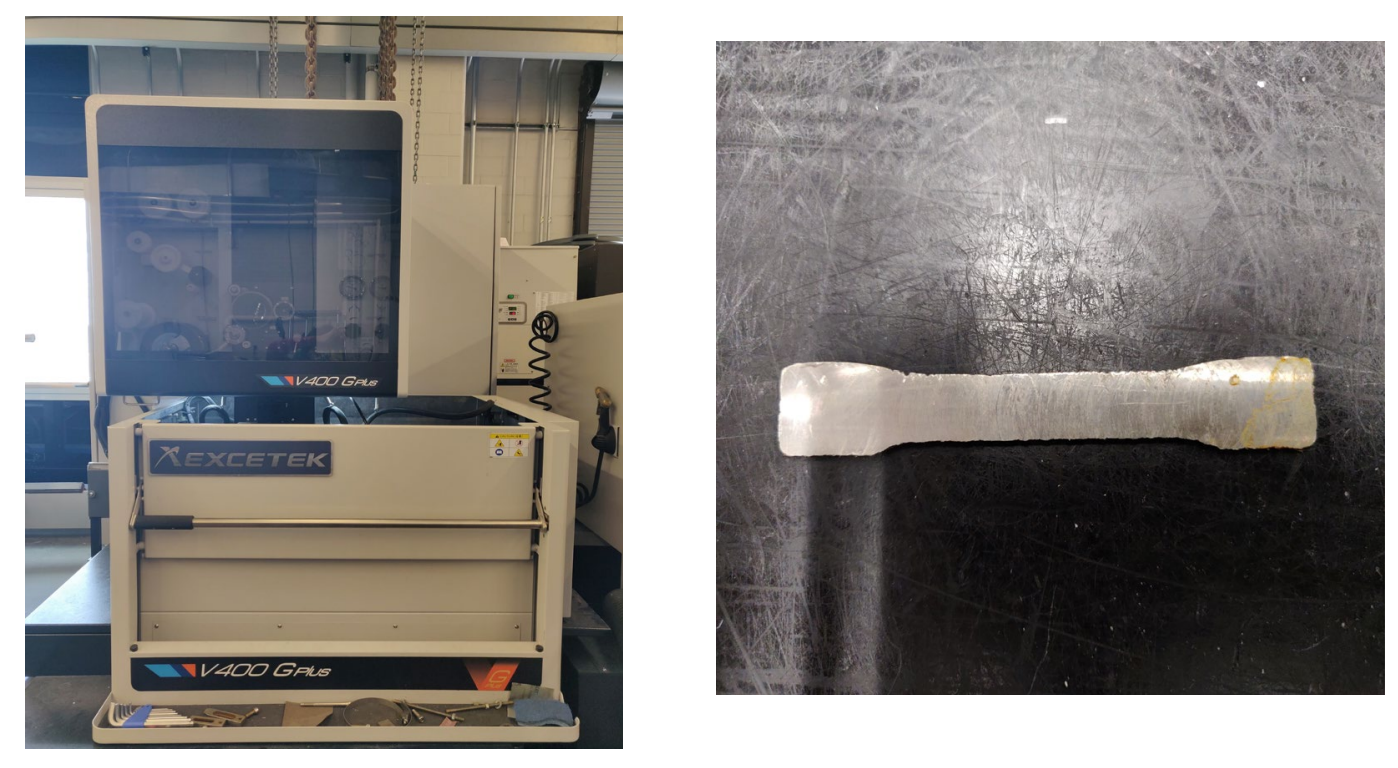

Figure 11: Electric Discharge Machining and the final dog bone specimen.

As we printed three dog bone specimens on each rectangular substrate, each specimen was first separated with substrate with the help of vertical bandsaw. Then each of the specimen were cut from its substrate with the height of $2 \mathrm{~mm}$ with the help of a wire EDM machine (VG plus series, EXCETEC Technologies Co. Ltd, Taiwan). A brass wire with small diameter of $0.23 \mathrm{~mm}$ cut the narrow width of $2 \mathrm{~mm}$ from the original specimen. The workpiece moved steadily around the wire so that it can have the accurate tool path. Numerical control was used during cutting to maintain the precision of the motion. While cutting, the wire continuously moved between from a supply and take in reels of wire to maintain the constant diameter of the electrode. Dielectric fluid was constantly flushed to wash away all the debris. Final finish was done to maintain the average 1.51 $\mathrm{mm}$ thickness of the specimen.

\subsubsection{Tensile Testing:}

Tensile testing is a good scheme to test the mechanical properties of materials i.e. Yield Strength, Ultimate Tensile Strength and Elongation (\%). In our work, the tensile test was performed on a universal testing machine (AGS-X, Shimadzu Co., Kyoto, Japan) with a $10 \mathrm{KN}$ capacity load cell to evaluate the tensile properties according to ASMT E8 The gauge length was $12.5 \mathrm{~mm}$ and testing speed was $1.5 \mathrm{~mm} / \mathrm{min}$.

The process is simple, to apply the load axially on the both sides till failure. Stress and strain are calculated from the recorded tensile loads and extensions. 

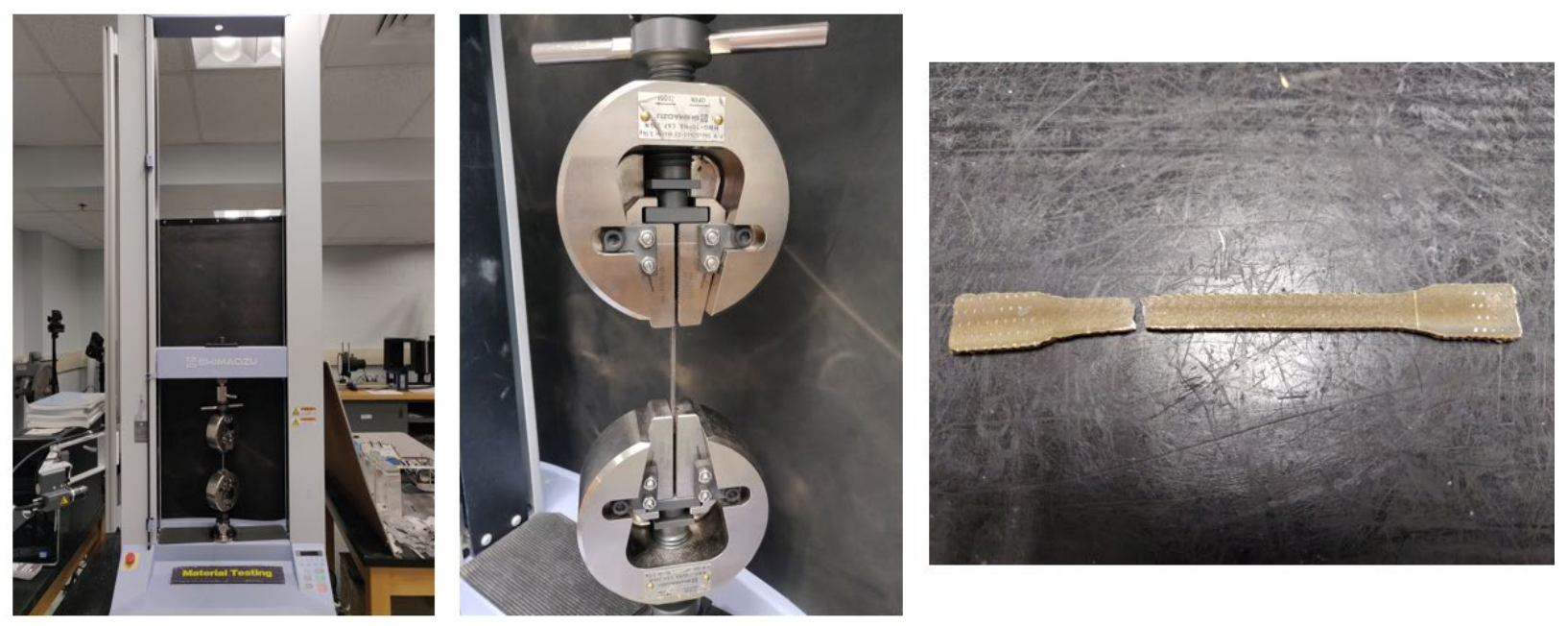

Figure 12: Universal testing Machine and dog bone specimen after failure.

For the process to start, first the cross-sectional area of each of the dog bone specimen is measured. Then the specimen is mounted to the both clamps of the machine tightly to avoid distortion while load is applied. The plastic deformation is visible while the experimentation is going. The graph is originated by the Trapezium software connected to the universal testing machine. The graphs we obtained have clearly visible all the engineering stress curves.

Our graphs contain defined four stress zones i.e. the elastic zone, yield strength, then maximum withstanding lead region and finally the fracture. The final data set is provided by the software to calculate the tensile strengths.

Yield Strength: Yield Strength obtained at the end of the elastic zone area of the stress-strain curve. It can be calculated by load at yielding $\boldsymbol{P}_{\boldsymbol{y}}$ divided by original area $A$.

$$
\sigma_{y}=\frac{P_{y}}{A}
$$

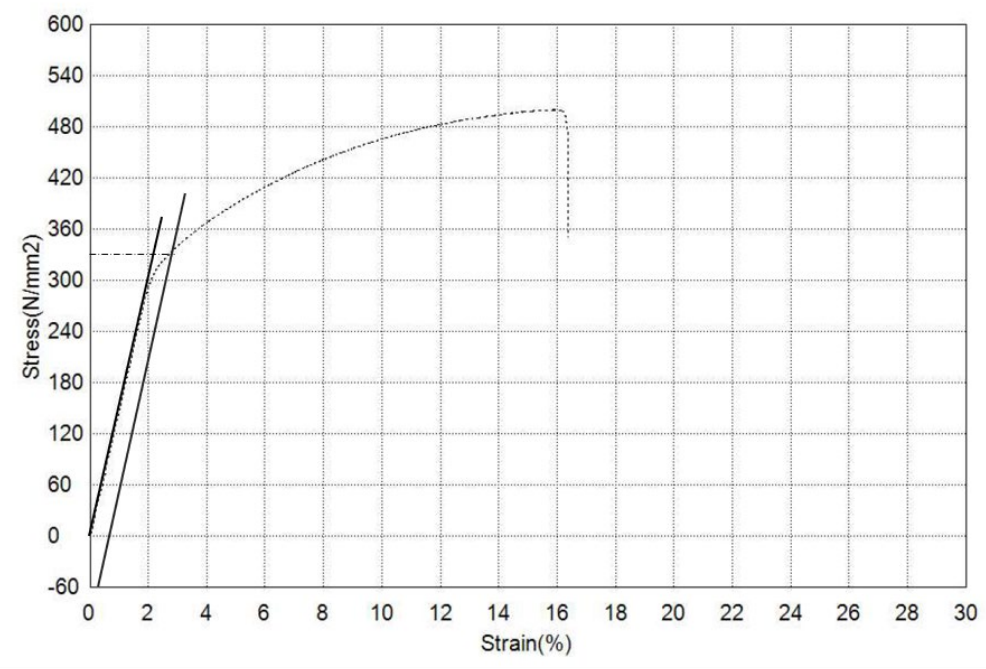

Figure 13: Yield Strength from the stress-strain curve graph (for sample \#6) 
Yield Strength is determined by drawing a straight parallel line to the stress-strain curve at $0.2 \%$ offset for metals like steel. In Figure 13, the interception point of the parallel line with the stressstrain curve is the respective yield strength. The graph is generated from Trapezium software. From the figure above, we get the yield strength of the sample \#6 is $326.4 \mathrm{MPa}$.

Ultimate Tensile Strength: Beyond yield point, the elastic zone end and further loading induces plastic deformation of the material. If the load is continuously applied, the stress-strain reaches the maximum point $\boldsymbol{P}_{\text {max }}$, from where the necking begins. This maximum point is called the ultimate tensile strength.

$$
\sigma_{u t s}=\frac{P_{\max }}{A}
$$

The ultimate tensile strength is calculated from the data file generated by Trapezium software connected to the universal tensile testing machine.

Elongation (\%): Elongation (\%) or reduction in area (\%) represents the tensile ductility. The equation of determining elongation (\%) is below:

$$
\text { Elongation (\%) }=\frac{\Delta L}{L_{0}} \times 100 \%
$$

Here, $L_{0}$ is the initial length of the sample and $\Delta L$ is the difference between the initial length and the elongated final length. Elongation (\%) is also calculated from the data file generated by Trapezium software connected to the universal tensile testing machine.

The average of the yield strength, ultimate tensile strength and elongation (\%) of the collected data from the five groups of $316 \mathrm{SS}$ fabricated parts are compared with the similar properties of raw 316 stainless steel (ASTM:A240) to verify that the printed parts have similar mechanical properties as the raw material.

Table 10: Comparison between the mechanical properties of printed and raw $316 \mathrm{SS}$

\begin{tabular}{|c|c|c|c|}
\hline Material & $\begin{array}{c}\text { Yield Strength } \\
\text { (MPa) }\end{array}$ & $\begin{array}{c}\text { Ultimate Tensile } \\
\text { Strength (MPa) }\end{array}$ & Elongation (\%) \\
\hline $\begin{array}{c}\text { Printed 316 SS by } \\
\text { L-DED }\end{array}$ & 310.5 & 544.6 & $32.4 \%$ \\
\hline Raw 316 SS & 207 & 504 & $40 \%$ \\
\hline
\end{tabular}

The mechanical properties obtained in our work are close to the ASTM standard values. (ASTM52900-15). The average yield strength that we obtained is 50\%, ultimate tensile strength is $8.06 \%$ improved but elongation (\%) is 19\% less than the ASTM standard. The fabricated parts behaved less ductile than the raw 316 stainless steel but possess more strength than the raw ones. 


\subsection{Data preprocessing}

We have collected three data set from the tensile testing i.e. Yield strength, Ultimate tensile strength and Elongation (\%). The data is preprocessed cause the data collected was in load (N) format. The cross-sectional area was calculated to determine the stress from the data. Elongation (\%) data was collected as the final length and then the percent elongation is calculated.

\subsubsection{Process Parameters}

We have considered four process parameters i.e. laser power, scanning speed, layer height and energy density for the input array $\left(\mathrm{X}_{0}\right)$ for three separate outputs $\left(\mathrm{Y}_{0}\right)$ Yield, Ultimate Tensile Strength and Elongation (\%) of the XGBoost model.

Laser Power: Laser power is one of the main parameters of the LENS processes. It controls both the amount the energy absorbed by the specimen and powder efficiency directly. (Padmanaban et. al, 2010)

With higher power, the heat is increased which melts more powder in the melt pool and the thickness of the specimen increases as a result. (Khalil et. al, 2016) The laser power ranged starting from $600 \mathrm{~W}$ to $1000 \mathrm{~W}$. All three mechanical properties are plotted against the laser power to check the trendline.

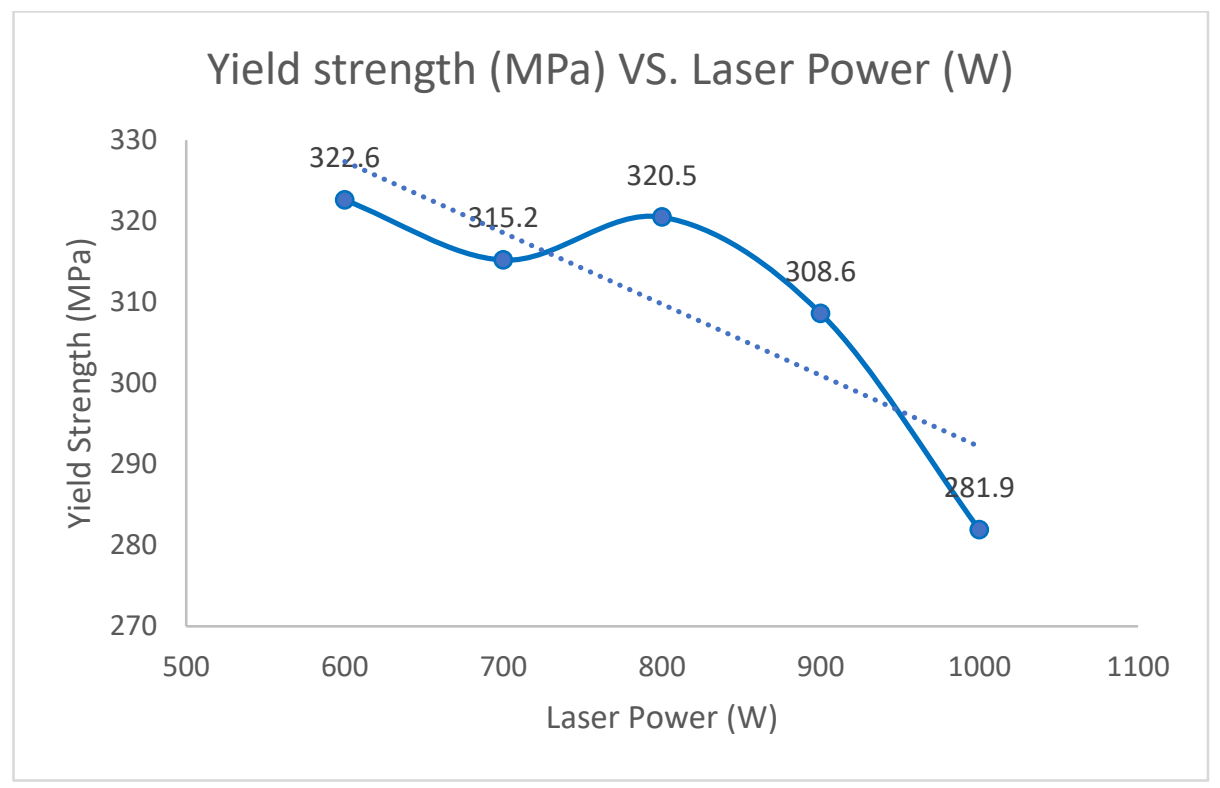

Figure 14: Yield Strength (MPa) VS. Laser Power (W) for $11 \mathrm{~mm} / \mathrm{sec}$ scanning speed 


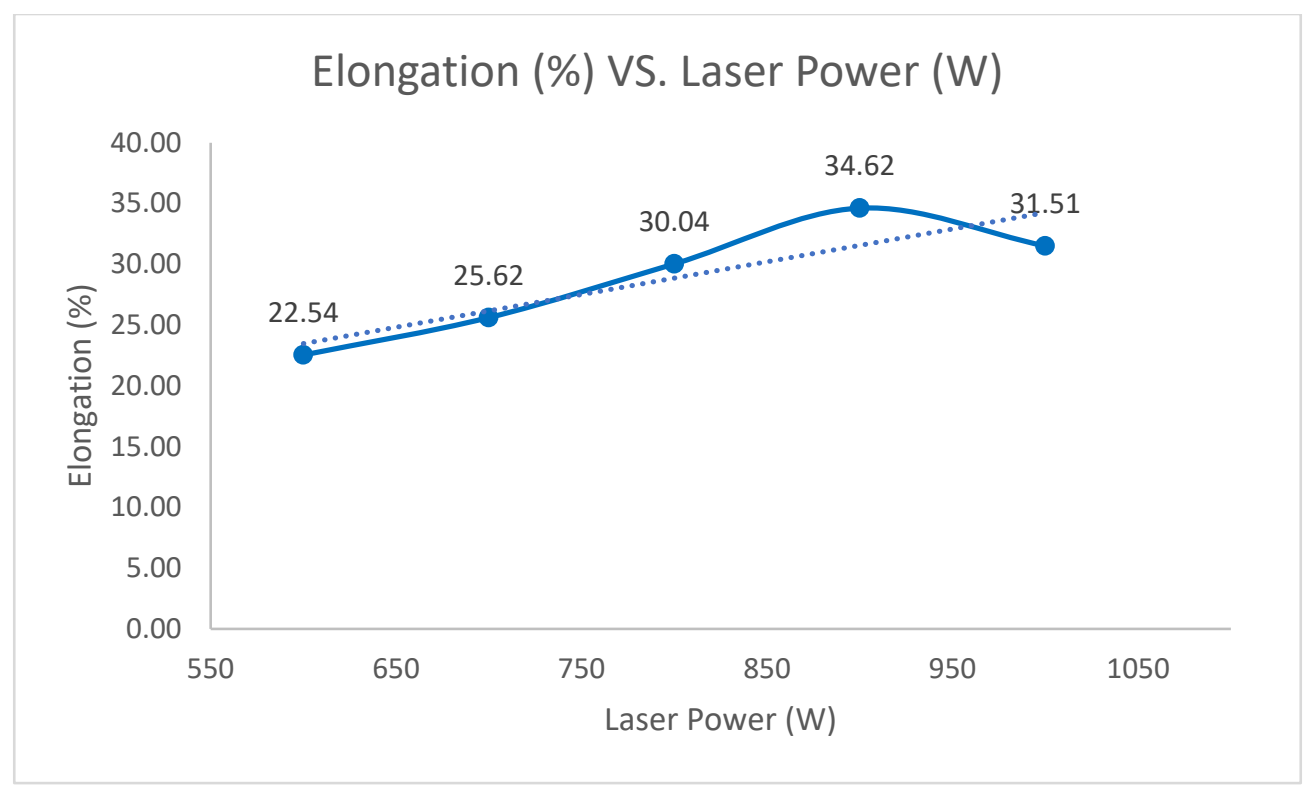

Figure 15: Elongation (\%) VS. Laser Power (W) for $11 \mathrm{~mm} / \mathrm{sec}$ scanning speed

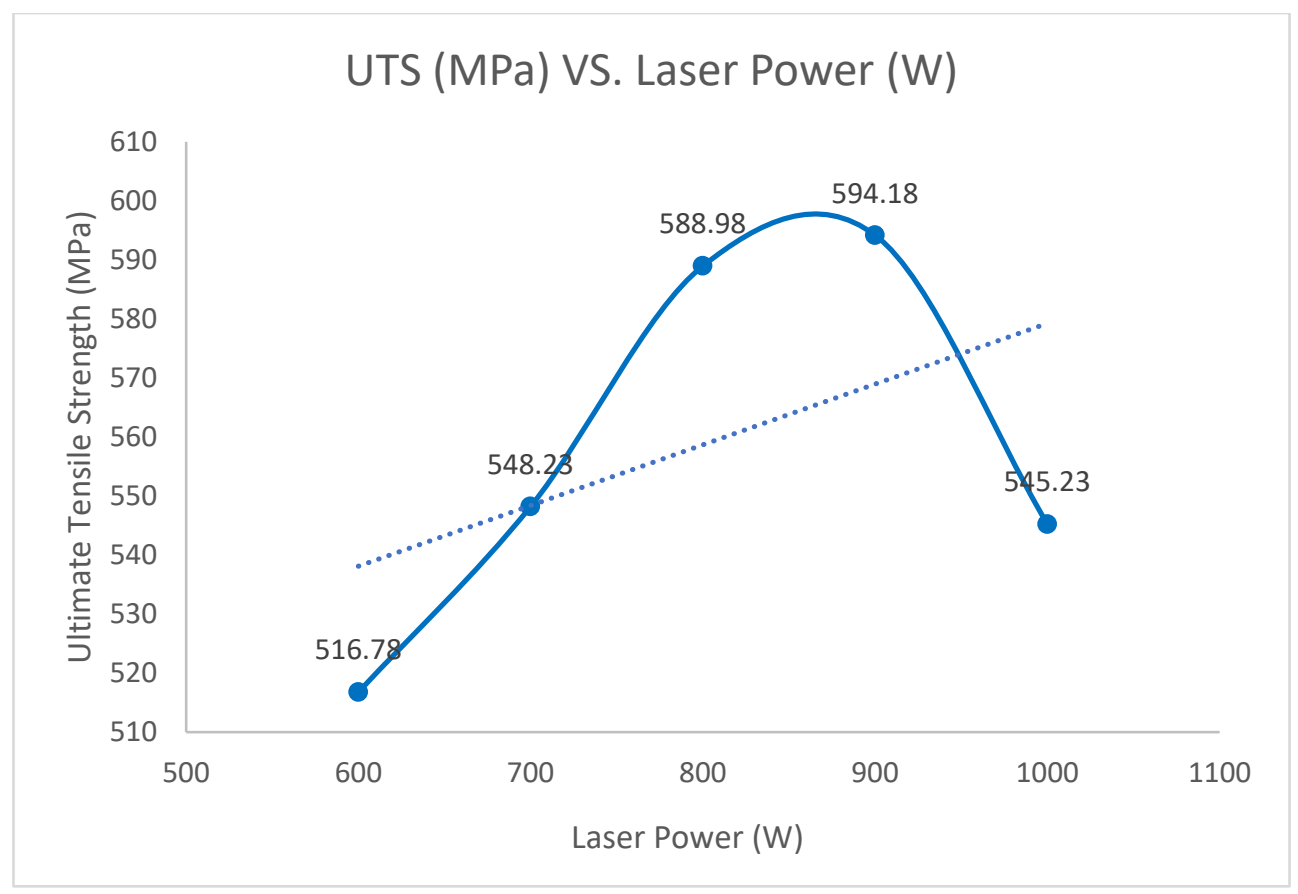

Figure 16: Ultimate Tensile Strength (MPa) VS. Laser Power (W) for $11 \mathrm{~mm} / \mathrm{sec}$ scanning speed.

From the figure 14, 15 and 16, yield strength $(\mathrm{MPa})$ has a descending trendline with the increase of the laser power whereas ultimate tensile strength (MPa) and elongation (\%) has an ascending trendline with the increase of laser power. But all the three curves dropped down at the maximum laser power $1000 \mathrm{~W}$. The yield strength obtained ranged from 278.6 MPa to 326.4 MPa. It's found that both the maximum and minimum yield strength $(\mathrm{MPa})$ are at 700 Watts. 
Energy Density: Energy density here is basically the effective energy provided by the laser. This is a parameter which defined how much energy is absorbed during melting of the surface of the substrate and the powder. (Shim et. al, 2016)

$$
E\left(J / m m^{2}\right)=\frac{P}{v D}
$$

Here, $\boldsymbol{P}$ is the laser power (W or $\mathrm{J} / \mathrm{s}), \boldsymbol{v}$ is the scanning speed $(\mathrm{mm} / \mathrm{sec}$ ) of the operating tool and $\boldsymbol{D}$ is the diameter $(\mathrm{mm})$ of the laser spot. In our work. $\boldsymbol{P}=[600,1000], \boldsymbol{v}=[8,12]$ and $\boldsymbol{D}=2 \mathrm{~mm}$ (constant).

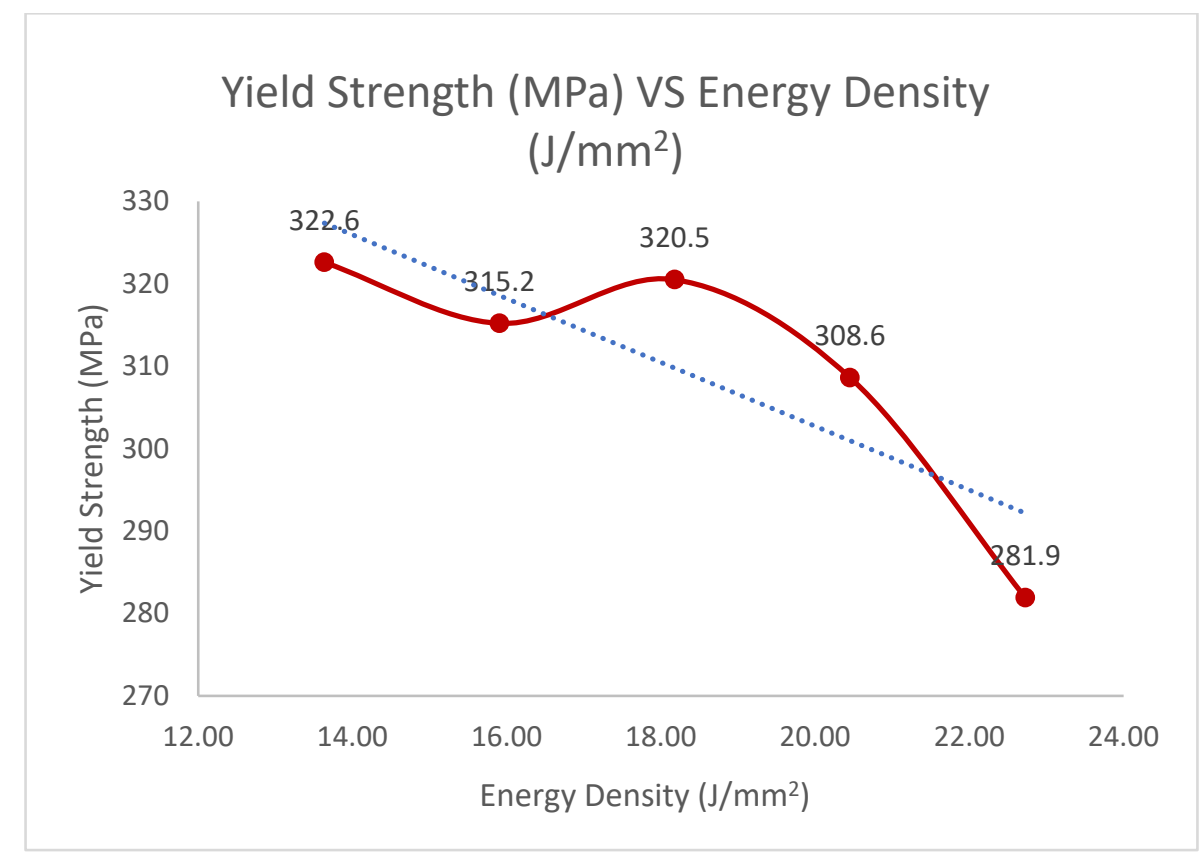

Figure 17: Yield strength (MPa) VS. energy density $\left(\mathrm{J} / \mathrm{mm}^{2}\right)$ for $11 \mathrm{~mm} / \mathrm{sec}$ scanning speed

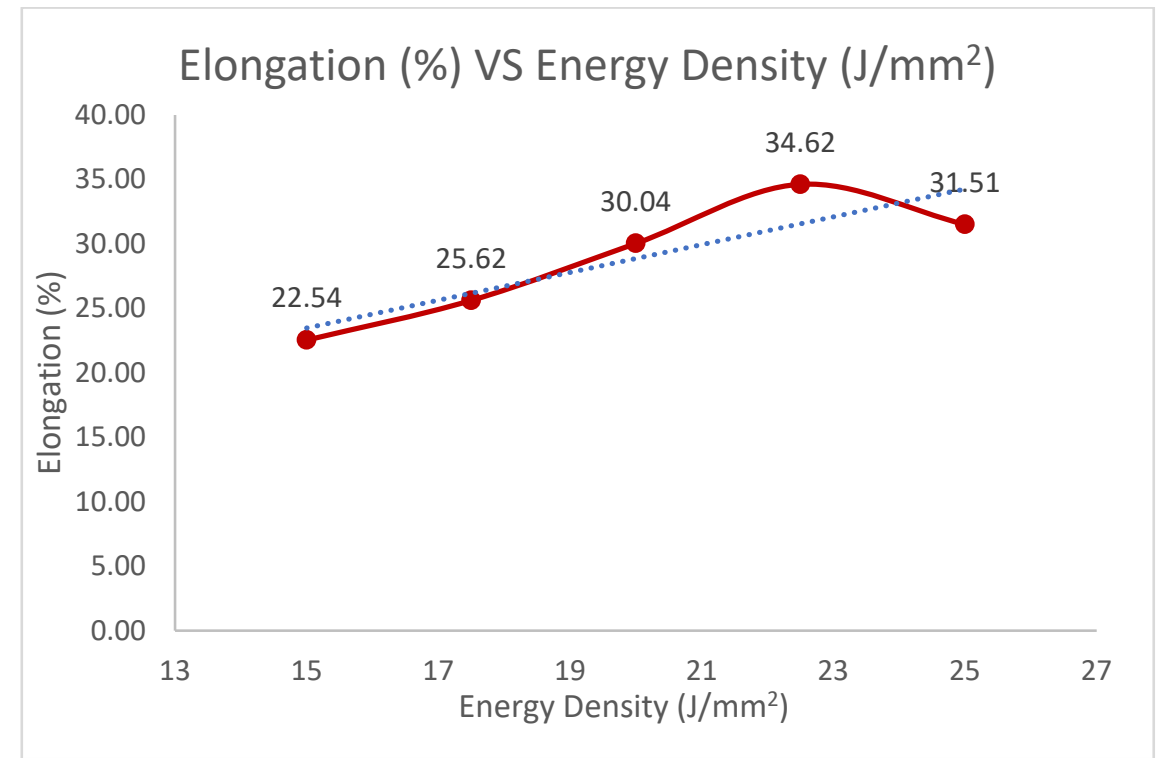

Figure 18: Elongation (\%) VS. energy density $\left(\mathrm{J} / \mathrm{mm}^{2}\right)$ for $11 \mathrm{~mm} / \mathrm{sec}$ scanning speed 


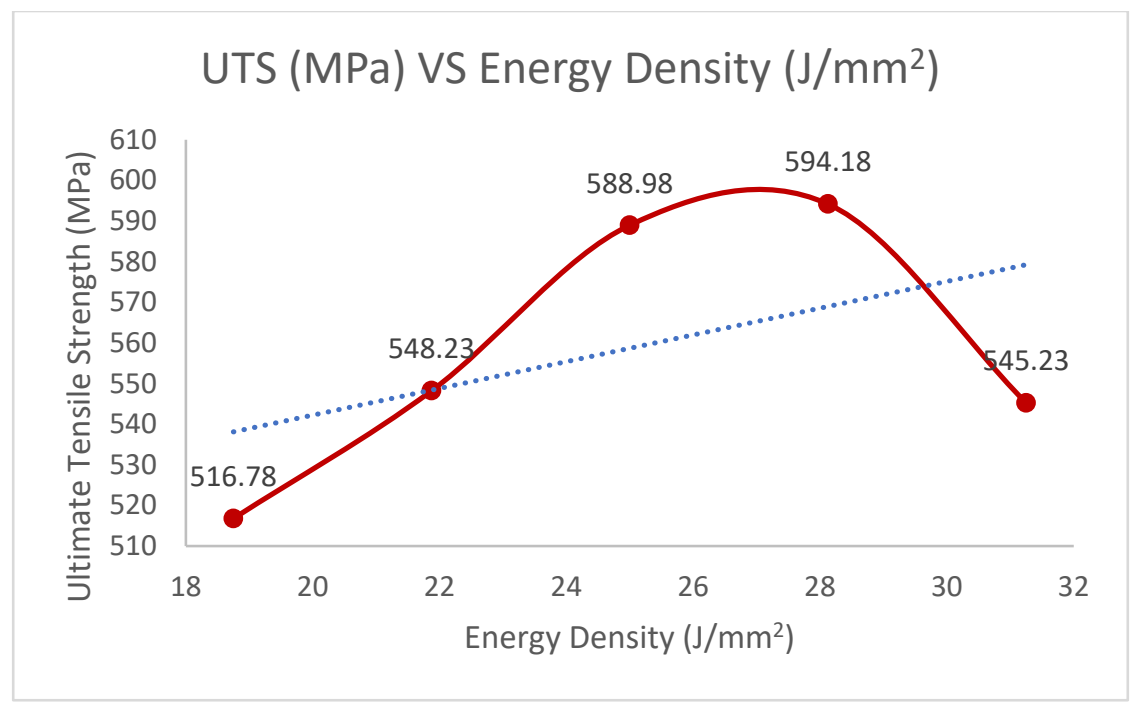

Figure 19: Ultimate tensile strength (MPa) VS. energy density $\left(\mathrm{J} / \mathrm{mm}^{2}\right)$ for $11 \mathrm{~mm} / \mathrm{sec}$ scanning speed.

From Figure 17,18 and 19, yield strength (MPa), elongation (\%) and ultimate tensile strength (MPa) showed the similar behavior around energy density $\left(\mathrm{J} / \mathrm{mm}^{2}\right)$ as laser power (W). These similarities are explained as energy density is the combined effect of laser power and scanning speed of the process. Therefore, the similarity of the trend to laser power is due the direct relationship of energy density with it.

Layer Height: Layer height affects not only the geometrical accuracies of the printed parts but also has influence on the mechanical properties as well. (Shim et al, 2016). In our work, we already mentioned there are five layers in each of the printed dog bone specimen. Here, we took the average single layer height for each of the specimen as one of the affecting parameters.

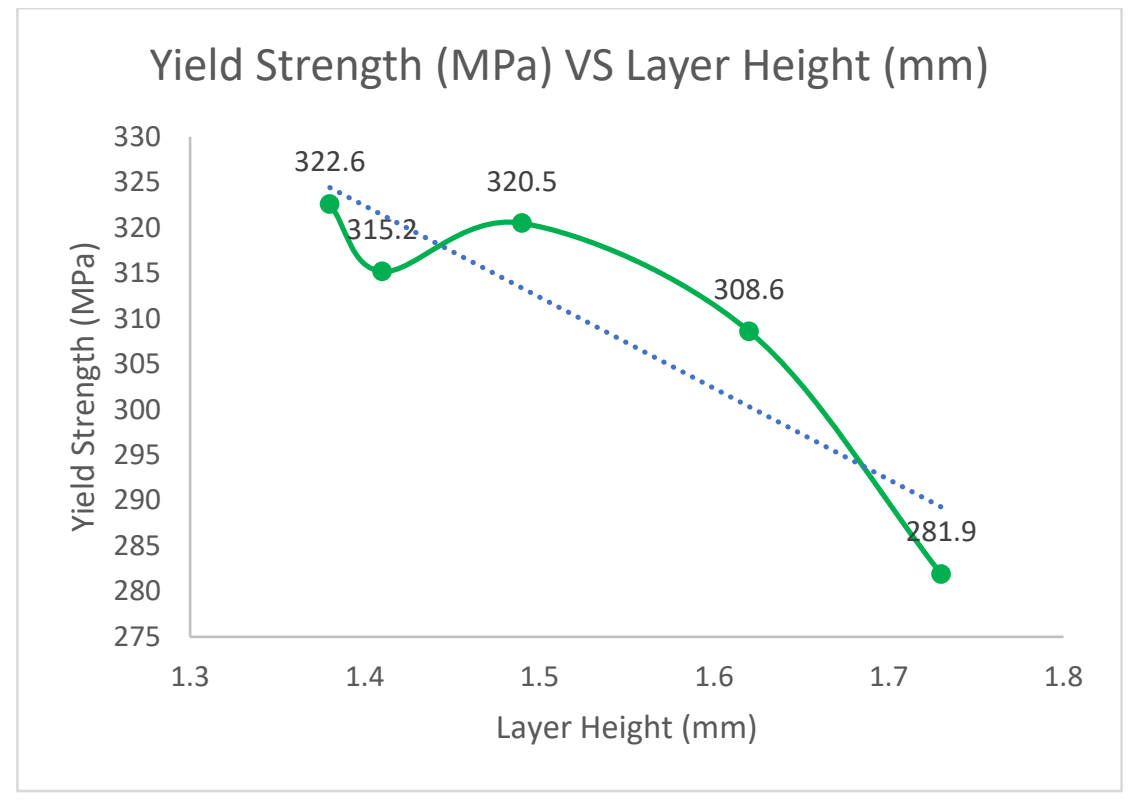

Figure 20: Yield Strength (MPa) VS. Layer Height (mm) for $11 \mathrm{~mm} / \mathrm{sec}$ scanning speed 


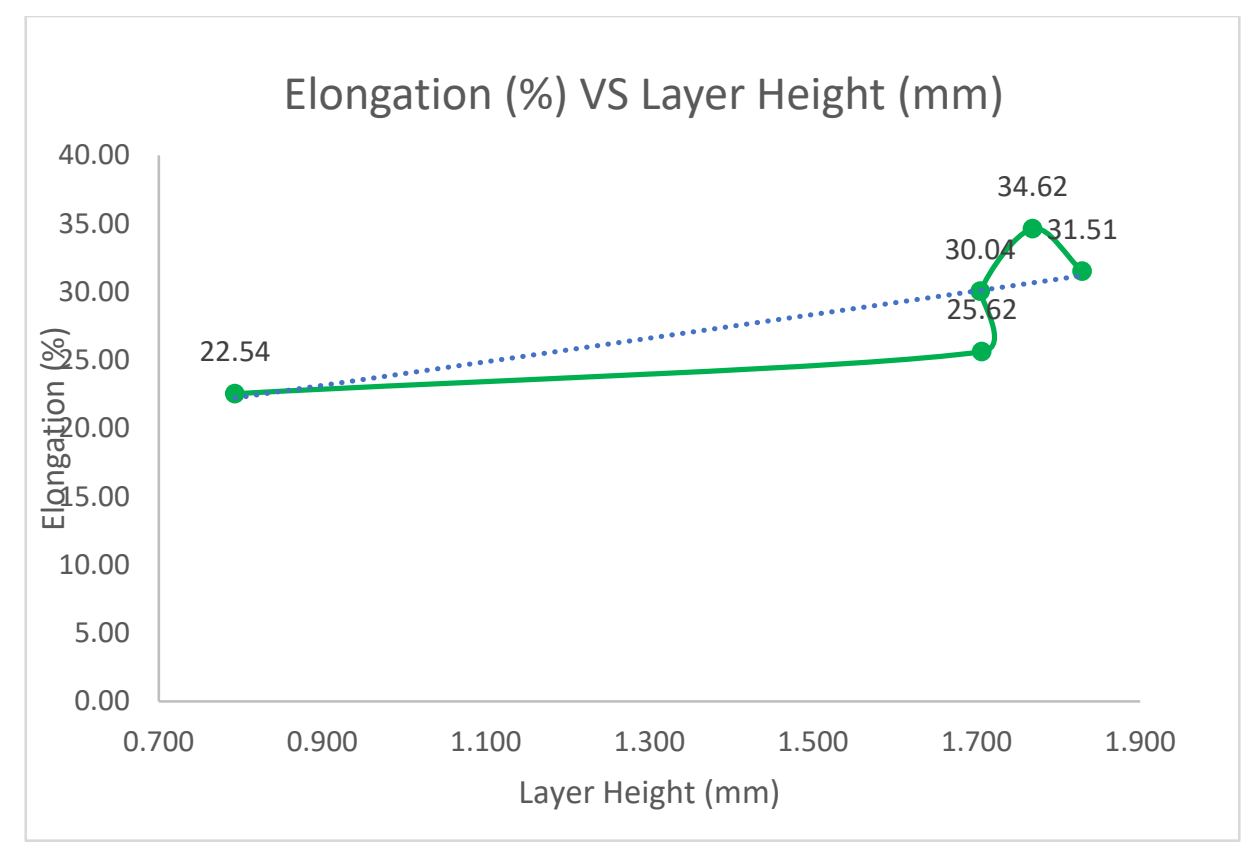

Figure 21: Elongation (\%) VS. Layer Height (mm) for $11 \mathrm{~mm} / \mathrm{sec}$ scanning speed.

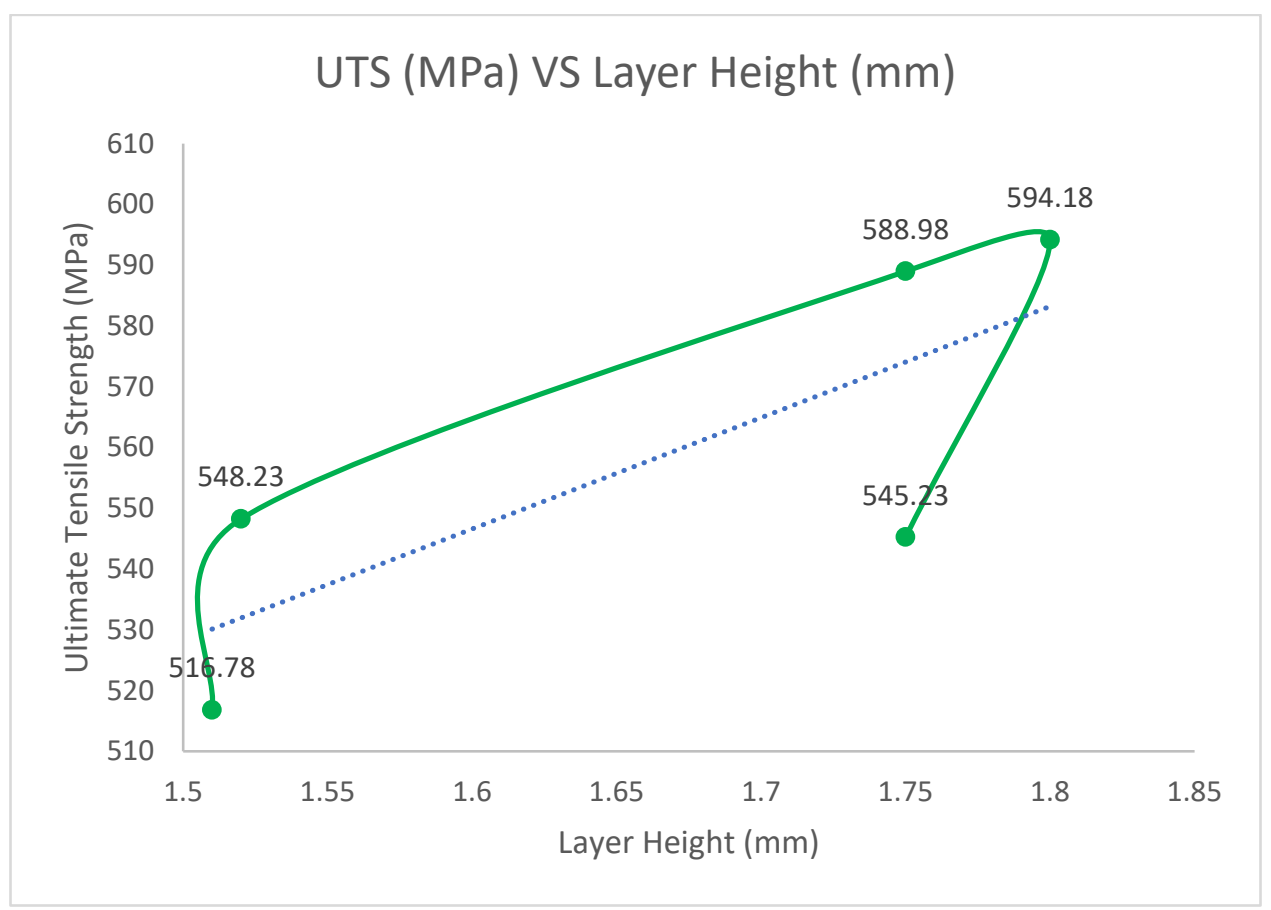

Figure22: Ultimate Tensile Strength (MPa) VS. Layer Height (mm) for $11 \mathrm{~mm} / \mathrm{sec}$ scanning speed.

In the Figure 20, 21 and 22, layer height ( $\mathrm{mm}$ ) seems to look has similar effect on yield strength (MPa), elongation (\%) and ultimate tensile strength (MPa) like other two process parameters. From all the nine graphs its evident that except for yield strength, rest of the tensile properties i.e. ultimate tensile strength and elongation (\%) are maximum at $900(\mathrm{~W})$ at $11 \mathrm{~mm} / \mathrm{sec}$ scanning speed. 
Lower laser power induces higher un-melted particles in the layers and also on the surface which is also responsible for shrinkage. So, the graph tends to rise with the rise of power but with higher laser power, there are higher chances of gas porosity which also means there is a higher thermal gradient. It induces higher residual stress which degrade the mechanical properties. Therefore, the graph tends to drop at the maximum laser power. As energy density is a combination of laser power and scanning speed, the graph behaved similar as laser power.

\subsubsection{Feature Selection and Importance}

One of the most notable problem in machine learning and data mining is to depict the data in terms of all the features. (Gao et. al, 2013) All the original features are not always proved to be beneficial for both regression and classification problems as some of the features are noises or redundant. (Liang et. al, 2020) And these redundant features affect the model's performance negatively (Güneş et. al, 2010) To optimize the trade-off between the better performance and computational cost, application of feature selection is suggested by machine learning users. Guyun et. al, 2002 formed F score as a feature selection for classification. Chen et. al, 2003 proposed a combined approach of SVM with F score. Polat et. al, 2009 introduced a new feature selection called kernel F score to remove redundant features from medical data. Güneş et. al, 2010 applied multi class F score approach to classification of obstructive sleep apnea syndrome. Gao et. al, 2013 used F score and extreme learning for lie detection. Song et. al, 2017 used FDA based F score for on UCI datasets. Liang et. al, 2020 compared F score with the DGI analysis of the single cell transcriptome of mRNA signature. Zhang et. al, 2019 showed the features importance of angle of incline, overlapping length and number of shells on the tensile strength of specimens fabricated by 3D cooperative printing. Over the period of numerous studies, machine learning algorithms have become the leading tools to use data driven predictive modelling. But the area of investigating the relationship among the parameters and tensile properties of L-DED specimens is still not well explored by ML users. The purpose of our work is to investigate and predict the correlation among tensile strength of the printed parts and the process parameters of Laser Based Directed Energy Deposition using machine learning. And in our work, we have employed XGBoost as this algorithm is a suitable choice for medium length tabular data.

Feature selection plays an important role in pattern recognition and machine learning. (Chen et. al, 2003) F-score is one of the most basic and effective method to assess the importance of each feature or parameters of the dataset. (Polat et. al, 2009) For statistical analysis of binary classification, F score is a measure of accuracy of the test. The score is calculated from the precision and recall. (Leon et. al, 2016) Precision is the number of true positive results divided by the number of all positive results with the wrong identified ones as well. Precision also is referred as positive prediction. Recall is the number of true positive results divided by the number of samples identified as positive and false negative. It indicates the sensitivity of the algorithm. (Leon et. al, 2016)

$$
\begin{gathered}
\text { Recall }=\frac{T P}{T P+F N} \\
\text { Precision }=\frac{T P}{T P+F P}
\end{gathered}
$$




$$
F(i)=\frac{\left(\overline{\mathrm{x}}_{\mathrm{i}}^{(+)}-\overline{\mathrm{x}}_{\mathrm{l}}\right)^{2}+\left(\overline{\mathrm{x}}_{\mathrm{i}}^{(-)}-\overline{\mathrm{x}}_{\mathrm{l}}\right)^{2}}{\frac{1}{\mathrm{n}_{+}-1} \Sigma_{\mathrm{k}=1}^{\mathrm{n}_{+}}\left(\mathrm{x}_{\mathrm{k}, \mathrm{i}}^{(+)}-\mathrm{x}_{\mathrm{i}}^{(+)}\right)^{2}+\frac{1}{\mathrm{n}_{-}-1} \Sigma_{\mathrm{k}=1}^{\mathrm{n}_{+}}\left(\mathrm{x}_{\mathrm{k}, \mathrm{i}}^{(-)}-\mathrm{x}_{\mathrm{i}}^{(-)}\right)^{2}}
$$

Here, where $\bar{x}_{1}, \bar{x}_{i}^{(+)}, \bar{x}_{i}^{(-)}$are the average of the ith feature of the whole, positive, and negative datasets respectively; $\mathrm{x}_{\mathrm{k}, \mathrm{i}}^{(+)}$is the ith feature of the $\mathrm{kth}$ positive instance; and is the $\mathrm{x}_{\mathrm{k}, \mathrm{i}}^{(-)}$ith feature of the kth negative instance. (Song et. al, 2016) The numerator indicates the difference between the positive and the negative sets, the denominator is the sum of the deviations from each feature set. Larger $\mathrm{F}$ value is a good indication of better discriminative power between the positive and negative sets. (Leon et. al, 2016)

There are three data set prepared with 25 set of yield strength, elongation (\%) and ultimate tensile strength. The test size is chosen $25 \%$ for the small size of the data. The samples are taken randomly by test_train_split function. The effects of the process parameters are then determined by XGBoost based on F score. The XGBoost library provides a built-in function to plot features by their importance. (Jason Brownlee, 2018) Four parameters i.e. Laser Power, Scanning Speed, Layer Height and Energy Density are considered to predict three tensile properties of the specimens separately. The three tensile properties are Yield Strength, Ultimate Tensile Strength and Elongation (\%). The features are automatically denoted according to their index in the input array (X), in our case, from f0 to f4. Laser power is denoted by f0, Scanning Speed by f1, Layer Height by f2 and Energy Density by f3.

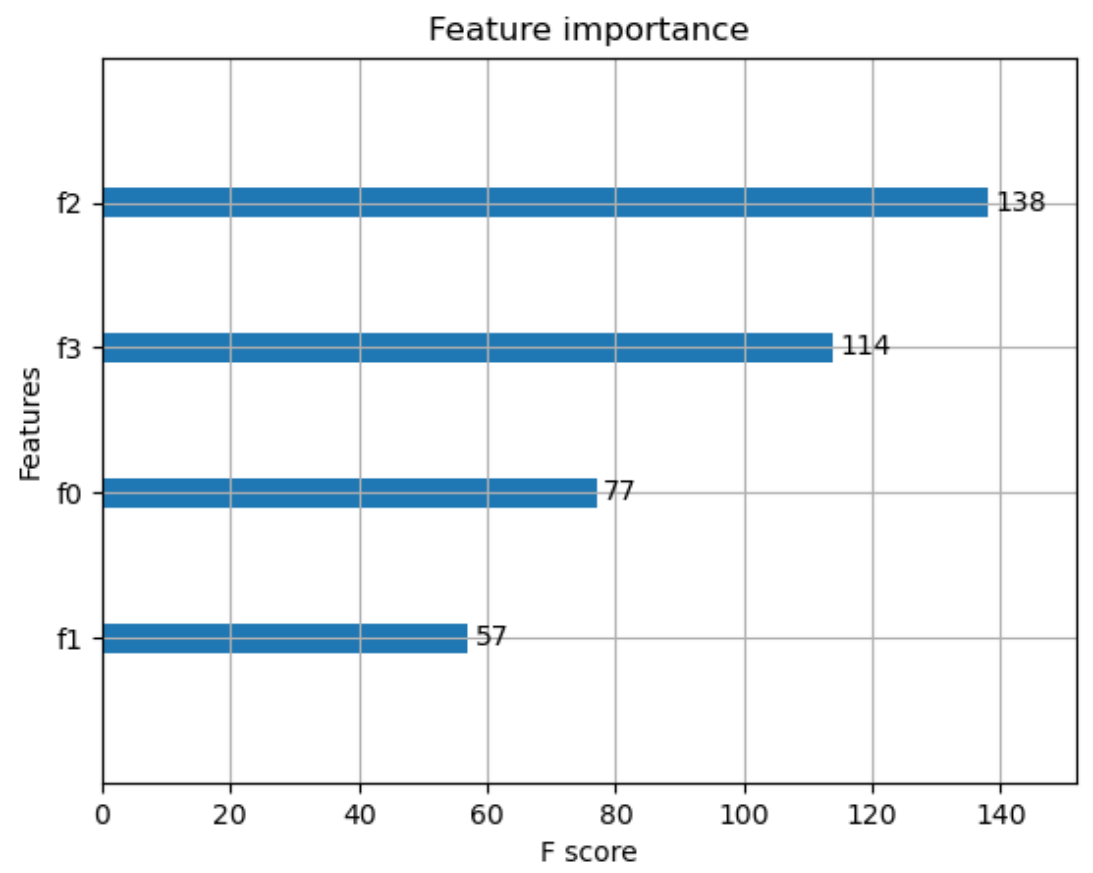

Figure 23: Feature Importance for Yield Strength (MPa) 


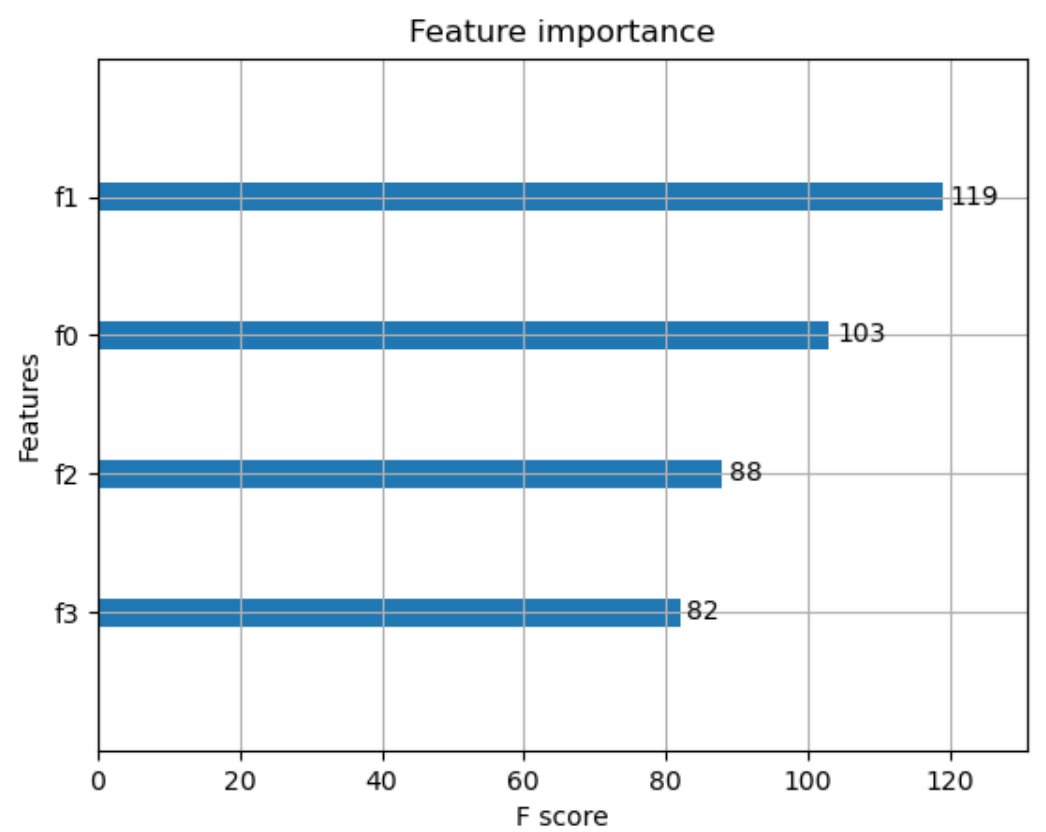

Figure 24: Feature Importance for Elongation (\%)

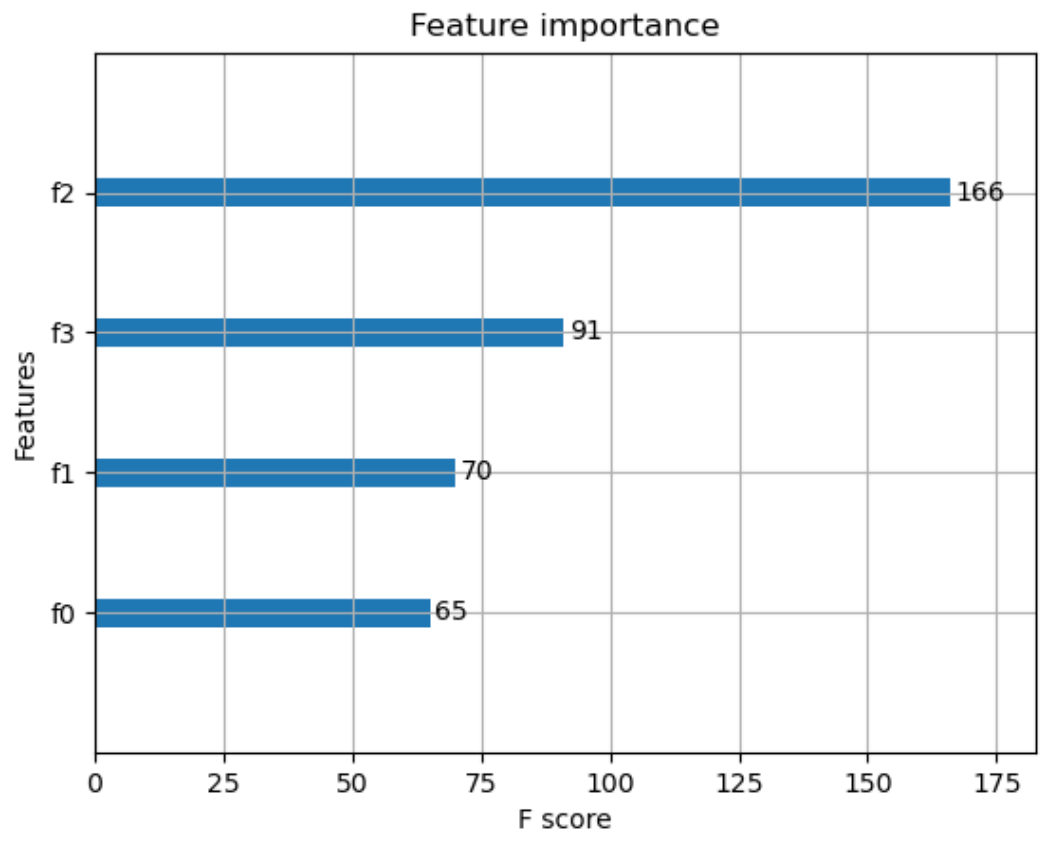

Figure 25: Feature Importance for Ultimate Tensile Strength (MPa)

In the graphs in Figure 23,24 and 25, layer height (f2) has the highest impact on the Yield Strength in our case and Scanning Speed (f1) the lowest. In case of ultimate tensile strength, layer height (f2) again has the maximum impact and laser power (f0) has the lowest. In case of elongation (\%), 
Scanning Speed (f1) has the maximum impact and energy density (f3) has the lowest from the other two graphs. 


\section{4. $\underline{\text { Result \& Discussion }}$}

\subsection{Prediction by XGBoost}

Every machine learning model follows a basic structure: Calling the built in functions from a machine learning library, load the data, define the input array $\left(\mathrm{X}_{0}\right)$ and the output array $\left(\mathrm{Y}_{0}\right)$, then define the training and testing split function, create the hyperparameter metrics as the param_grid and chose the optimization tool, next part is to call the prediction function then define the performance evaluation metrics. Graphical representation of the prediction is optional, depends on the users.

In our work we have used Scikit learn as the library and PyCharm 2020.2 version as the writing module. XGBoost has built-in functions in Scikit learn library. Both numpy and pandas library were called for the data loading and graphical representation purpose. The data files are loaded in .CSV format. If we recall Table 2: The summary of the architecture of the model, the param_grid created with the max_depth, n_estimators, learning_rate, min_child_weitgh, reg_alpha, reg_lamda and random_state.

Hyperparameter tuning is completed using RandomizedSearch cross validation and XGRegressor as the main fitting function are called from the sk.learn_model selection. The predictions are fitted against the randomly selected test data. The plots are created comparing the predicted and test data below:

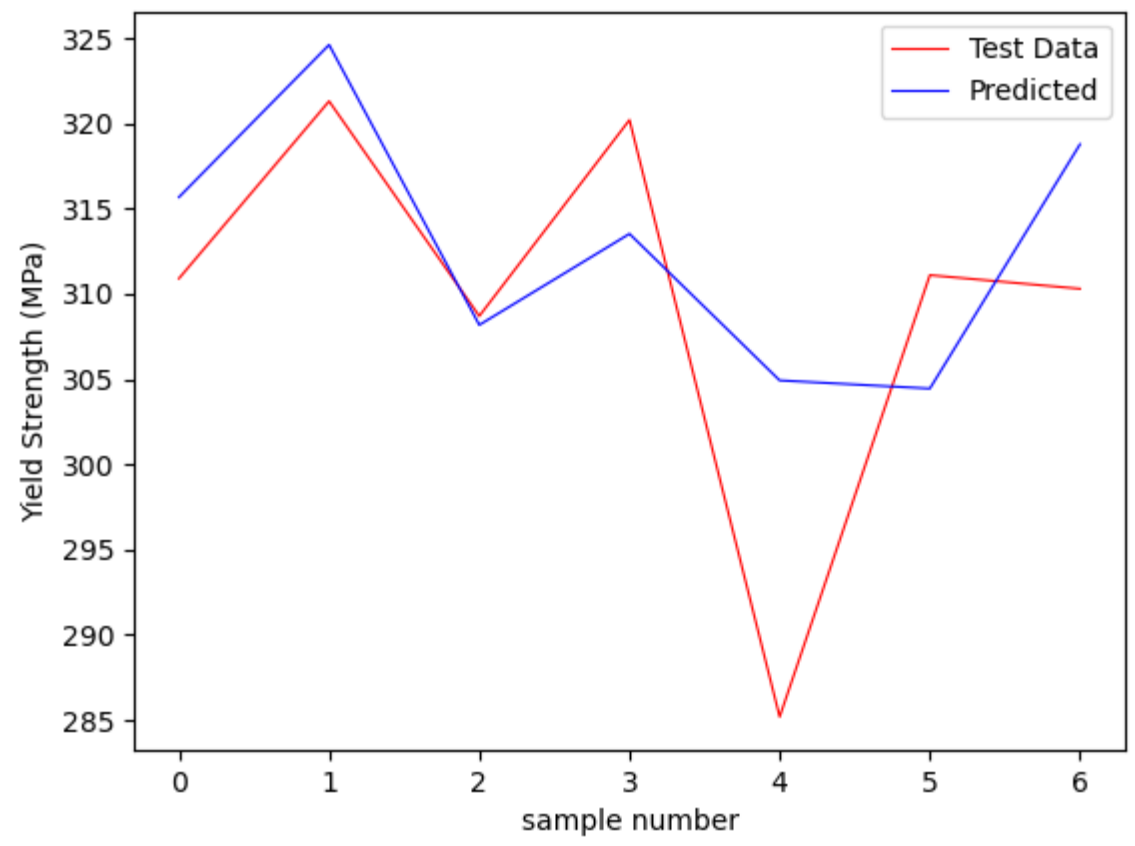

Figure 26: The predicted and test data for Yield Strength (MPa) by XGBoost 


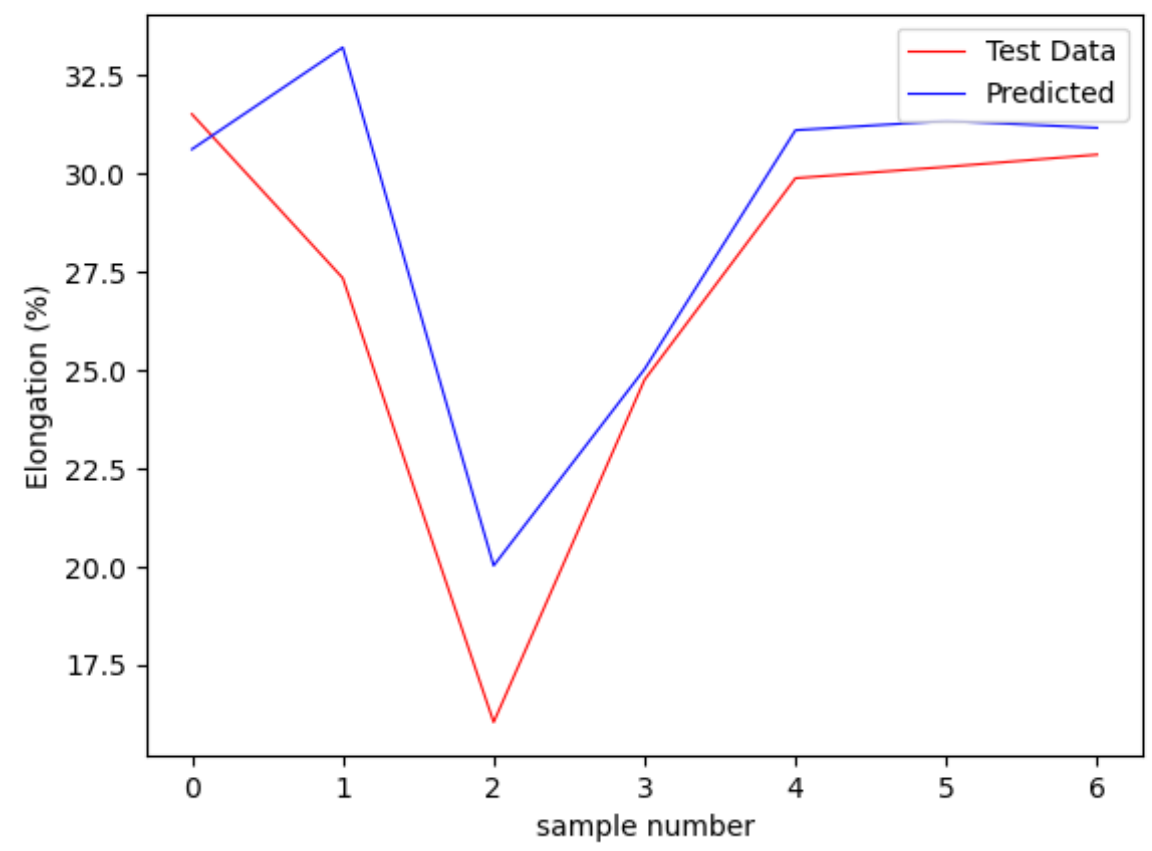

Figure 27: The predicted and test data for Elongation (\%) by XGBoost

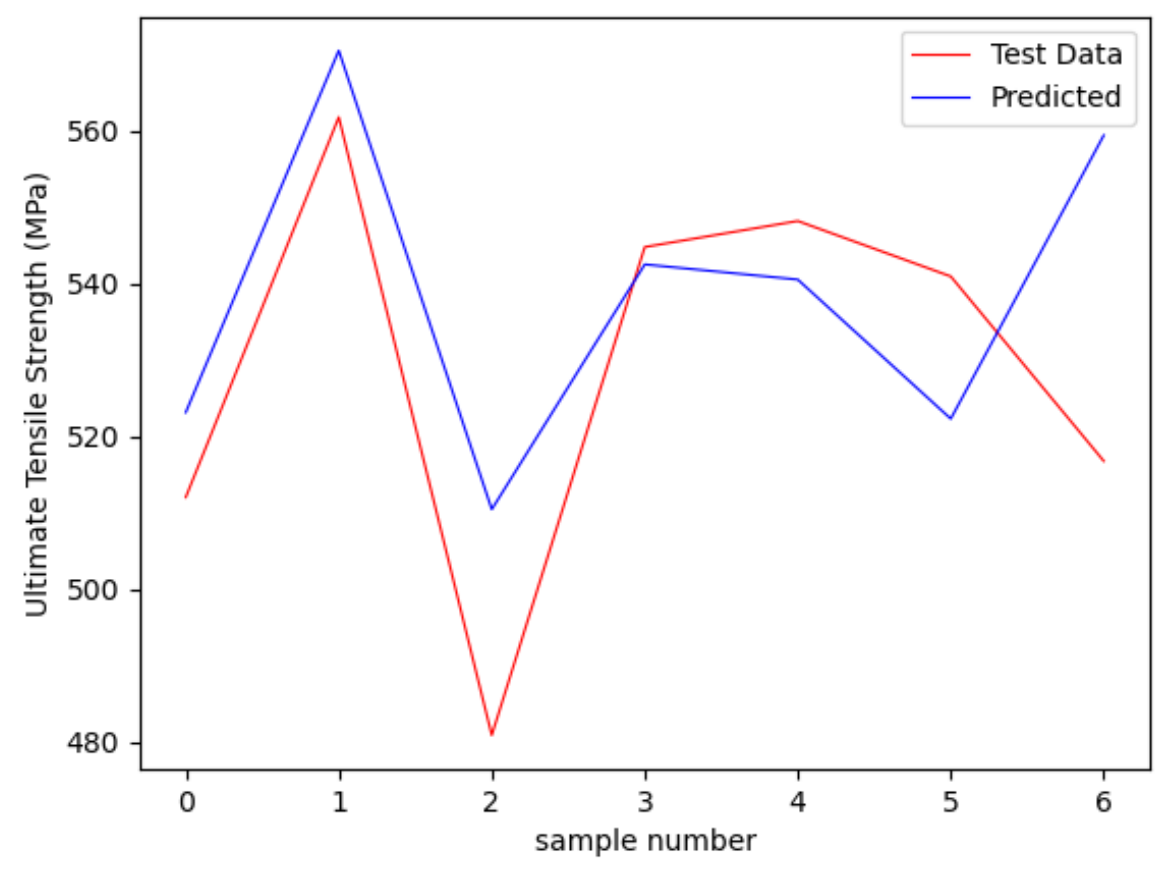

Figure 28: The predicted and test data for UTS (MPa) by XGBoost 
The graphs in Figure 26,27 and 28 are representation of how well the model fitted to the data and smaller residuals are observed in few points which is a good indicator of the performance accuracy of the model. To get a clearer idea of the accuracy, performance evaluation is performed on the predicted and test data.

\subsubsection{Performance Evaluation}

As we mentioned before, performance evaluation is an indicator of the model's accuracy and precision of predicting the output. For regression modeling, root mean square error, co-efficient of determination, relative error are suitable statistical metrics for this purpose.

Root Mean Square Error (RMSE): RMSE has been a standard statistical metric to measure the model performance. (Chai et. al, 2014). To calculate RMSE, we assume there are n number of samples and unbiased error in the prediction and test data. Smaller RMSE value indicates better performance of the model.

$$
R M S E=\sqrt{\sum_{i=1}^{n} \frac{\left(\widehat{y}_{l}-y_{i}\right)^{2}}{n}}
$$

Relative Error (RE): Relative error is the ratio of absolute error and the real measurement taken. This is basically used to explain how accurate the prediction was compared to the real value.

$$
R E=\frac{1}{n}\left|\sum_{i=1}^{n} \frac{\hat{y}_{i}-y_{i}}{y_{i}}\right| \times 100 \%
$$

Here, $\widehat{\boldsymbol{y}}_{\boldsymbol{l}}=$ predicted values, $\boldsymbol{y}_{\boldsymbol{i}}=$ observed values, $\overrightarrow{\boldsymbol{y}}_{\boldsymbol{i}}=$ mean of the observed values.

Co-efficient of determination $\mathbf{R}^{2}$ : Coefficient of determination explains how much of the data has fitted the regression model. It is denoted by $\mathrm{R}^{2}$. Usually closer the value to 1 indicates the model has fitted the data well but not always a higher value is a good indication of better fitted model.

$$
R^{2}=1-\frac{\sum_{i=1}^{n}\left(\widehat{y}_{l}-y_{i}\right)^{2}}{\sum_{i=1}^{n}\left(\vec{y}_{i}-y_{i}\right)^{2}}
$$

Here, $\widehat{\boldsymbol{y}}_{\boldsymbol{l}}=$ predicted values, $\boldsymbol{y}_{\boldsymbol{i}}=$ observed values, $\overrightarrow{\boldsymbol{y}}_{\boldsymbol{i}}=$ mean of the observed values.

The performance evaluation metrics of our XGBoost model are computed using the sk.learn_metrics. For RMSE, mean_sqaure_error, for coefficient of determination r2_score is called from the respective library. The relative error calculation is manually imported to the metrics. 
Table 11: The performance evaluation metrics for XGBoost

\begin{tabular}{|c|c|c|c|}
\hline Test & RMSE & $\mathbf{R}^{2}$ & $\mathbf{R E}$ \\
\hline Yield Strength (MPa) & 6.09 & 0.71 & $1.11 \%$ \\
\hline Ultimate Tensile Strength (MPa) & 11.89 & 0.65 & $3.23 \%$ \\
\hline Elongation (\%) & 2.64 & 0.73 & $0.83 \%$ \\
\hline
\end{tabular}

The RMSE we obtained for the three outputs in the Table 11, indicates the aggregated means of the deviation of the predicted values from the test data. The deviation is highest for ultimate tensile strength (MPa) (11.89) and lowest for the elongation (\%) (2.64), which indicates how well the model fitted to the training data of ultimate tensile strength (MPa) and elongation (\%). Coefficient of determination $\mathrm{R}^{2}$ values meant here is the model fitted to the $71 \%$ of the yield strength (MPa) data, $65 \%$ of the ultimate tensile strength (MPa) data and $73 \%$ of the elongation (\%) data. From the relative errors results, we interpret that the relative uncertainty of the prediction to the actual values for yield strength is $1.11 \%$, for ultimate tensile strength (MPa) is $3.23 \%$ and for elongation $(\%)$ is $0.83 \%$.

\subsubsection{Confidence Interval by Bootstrap}

Bootstrap method is a resampling technique to estimate statistics on a population by sampling with replacement. Samples are formed by drawing them from a large data set one at a time and then returning them to the data after they have been selected. This allows the given observation to be included in the small sample more than once.

The procedure starts with choosing the bootstrap sample size and for each bootstrap sample, drawing a sample with replacement with chosen size. Then fit the model on the data and the estimate the skill of the model. Then calculate the mean of the sample of model skill estimates.

The sample will be selected with replacement using resample() function from sk.learn. Any row that is not included in the sample are retrieved and used as the test data. Next a XGBoost_regressor fit the data and evaluated the test set. The RMSE is calculated and then added to the list RMSE calculated from all the bootstrap samples. Once the scores are collected, a histogram is created to exhibit how the distribution of the score.

The histogram of confidence interval of yield strength (MPa), ultimate tensile strength (MPa) and elongation (\%) are below: 


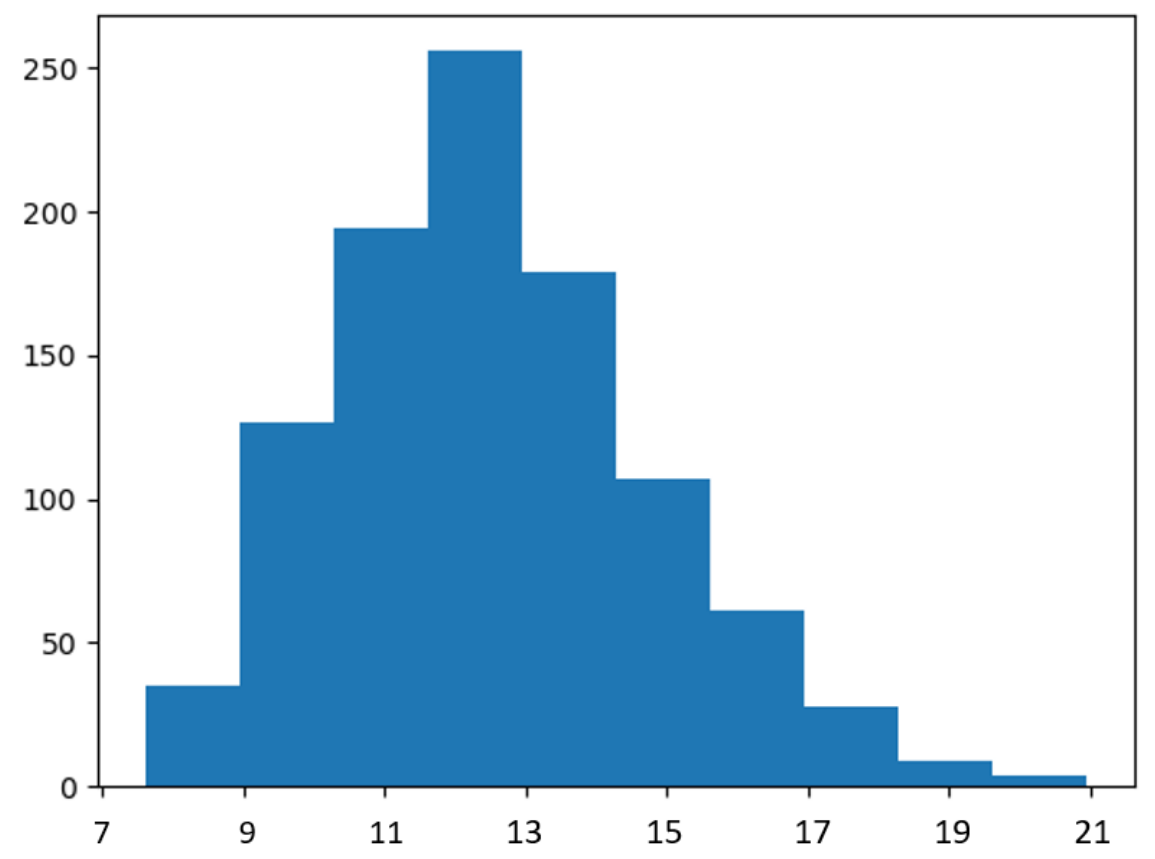

Figure 29: The histogram of distribution of RMSE of yield strength (MPa) by bootstrap

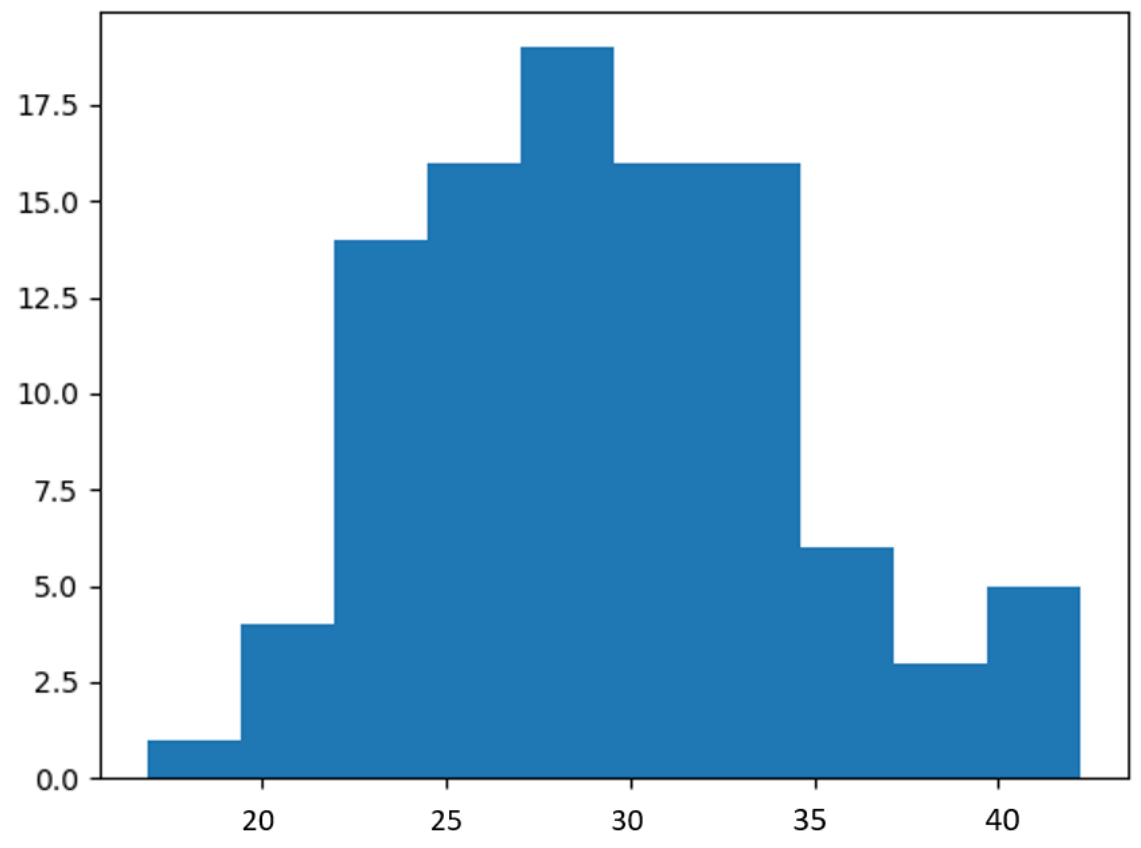

Figure 30: The histogram of distribution of RMSE of ultimate tensile strength (MPa) by bootstrap 


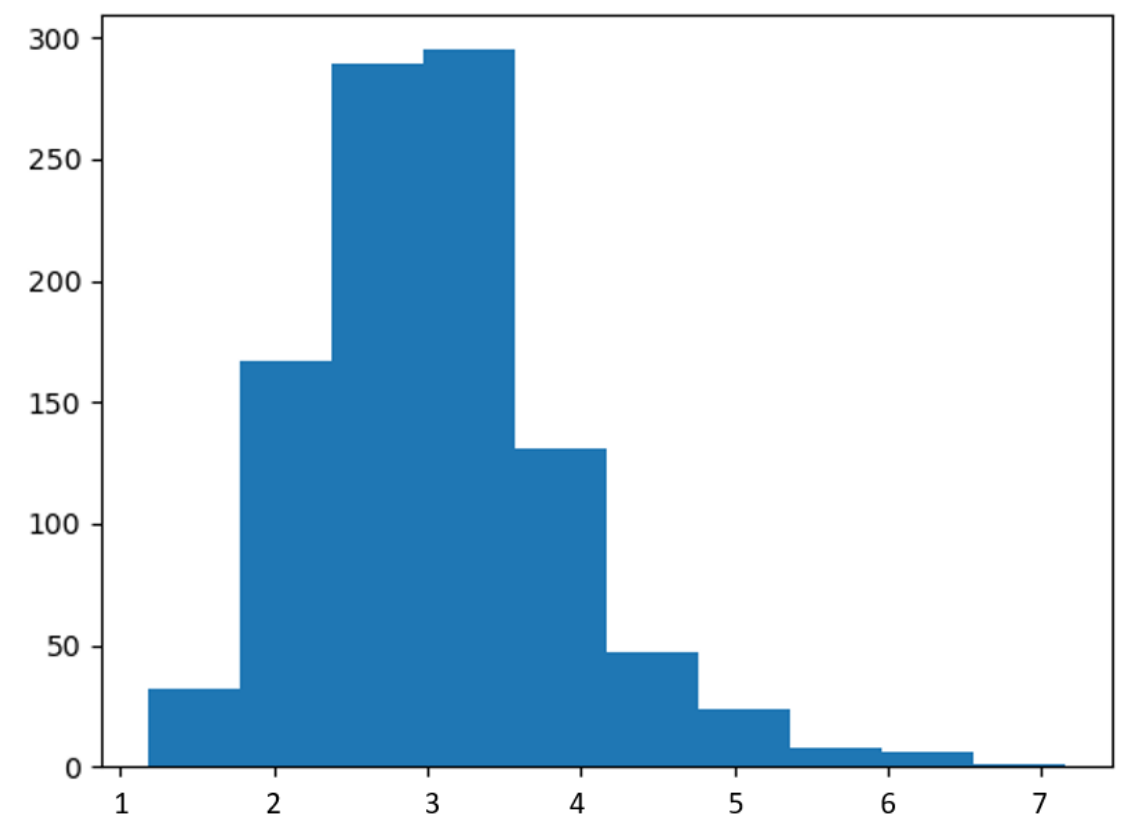

Figure 31: The histogram of distribution of RMSE of elongation (\%) by bootstrap

The distribution graphs we obtained from the three tensile strength properties are gaussian in nature, slightly right skewed for both yield strength and elongation (\%) and symmetrical for UTS. In case of yield strength (MPa), there is a 95\% likelihood that the confidence interval 9.01 and 15.99 covers the true skill of the model. For ultimate tensile strength (MPa), there is a $95 \%$ likelihood that the confidence interval 15.76 and 36 and for elongation (\%) 1.98 and 4.64 cover the true skill of the model.

\subsection{Comparison with the statistical models}

The performance accuracy of our XGBoost model is compare to the performance of two conventional statistical methods. First the linear regression model of the three tensile properties are built in order to check the relationship between the explanatory variables with the response variables. Then Ridge Regression is employed to the data for better comparison.

\subsubsection{Linear Regression}

Linear regression Linear Regression is performed to establish the relationship between the variables i.e. laser power, scanning speed, energy density, layer height with response variables i.e. yield strength, ultimate tensile strength and elongation (\%). The coefficient obtained indicates the effect of the variables on the response. T value and $\mathrm{P}$ value describe the statistical significance of individual variables. The ratio of coefficient and the respective standard error is denoted by $\mathrm{T}$ values. P value explains the probability against the null hypothesis of the test. (Zhang et.al, 2019)

ANOVA is a traditional statistical method to test research hypothesis. The degree of freedom represents the amount of information used in the analysis. The variation of the response which can be explained by the variables are represented by adjusted sum (adj ss). The Adj SS of error 
represents the variation of the response which can't be explained by the variable. Adjustable mean square addresses the variation with degree of freedom. F value explains the statistical importance of a variable and is calculated by the ratio of Adj MS of the respective variable to Adj MS of the error. (Zhang et. al, 2019). Linear regression and ANOVA analysis were performed on the three data set by Minitab 14 .

\subsubsection{Regression Analysis: Elongation (\%) versus Power (W), Scanning Speed (mm/sec), Layer Height (mm), Energy Density $\left(\mathrm{J} / \mathrm{mm}^{2}\right)$}

\section{Regression Equation}

Elongation (\%) $=-20.9-0.0103$ Power $(\mathrm{W})+3.00$ Scanning Speed $(\mathrm{mm} / \mathrm{sec})+3.77$

Height $(\mathrm{mm})+1.06$ Energy Density $(\mathrm{J} / \mathrm{mm} 2)$

Table 12: Coefficients Regression analysis of Elongation (\%)

\begin{tabular}{|c|c|c|c|c|c|}
\hline Term & Coef & SE Coef & T-Value & P-Value & VIF \\
\hline Constant & -17.4 & 21.1 & -0.83 & 0.419 & \\
\hline Power $(\mathrm{W})$ & -0.0086 & 0.0253 & -0.34 & 0.739 & 34.01 \\
\hline Scanning Speed $(\mathrm{mm} / \mathrm{sec})$ & 2.51 & 2.06 & 1.22 & 0.238 & 22.67 \\
\hline Layer Height $(\mathrm{mm})$ & 3.14 & 3.30 & 0.95 & 0.352 & 1.45 \\
\hline Energy Density $\left(\mathrm{J} / \mathrm{mm}^{2}\right)$ & 0.884 & 0.976 & 0.91 & 0.376 & 55.74 \\
\hline
\end{tabular}

Table 13: Analysis of Variance of Elongation (\%)

\begin{tabular}{|c|c|c|c|c|}
\hline Source & DF & Adj SS & Adj MS & F-Value \\
\hline Regression & 4 & 170.651 & 42.663 & 4.54 \\
\hline Power $(\mathrm{W})$ & 1 & 1.076 & 1.076 & 0.11 \\
\hline Scanning Speed $(\mathrm{mm} / \mathrm{sec})$ & 1 & 13.872 & 13.872 & 1.48 \\
\hline Layer Height $(\mathrm{mm})$ & 1 & 8.522 & 8.522 & 0.91 \\
\hline Energy Density $\left(\mathrm{J} / \mathrm{mm}^{2}\right)$ & 1 & 7.700 & 7.700 & 0.82 \\
\hline Error & 20 & 187.886 & 9.394 & \\
\hline Total & 24 & 358.537 & & \\
\hline
\end{tabular}

\subsubsection{Regression Analysis: UTS (MPa) versus Power (W), Scanning Speed (mm/sec), laver Height (mm), Energy Density $\left(\mathrm{J} / \mathrm{mm}^{2}\right)$}

\section{Regression Equation}

UTS $(\mathrm{MPa})=9-0.478$ Power $(\mathrm{W})+44.5$ Scanning Speed $(\mathrm{mm} / \mathrm{sec})+53.5$ layer Height $(\mathrm{mm})+19.19$ Energy Density $(\mathrm{J} / \mathrm{mm} 2)$ 
Table 14: Coefficients of regression analysis of UTS (MPa)

\begin{tabular}{|c|c|c|c|c|c|}
\hline Term & Coef & SE Coef & T-Value & P-Value & VIF \\
\hline Constant & 9 & 191 & 0.05 & 0.962 & \\
\hline Power $(\mathrm{W})$ & -0.478 & 0.229 & -2.09 & 0.050 & 34.01 \\
\hline Scanning Speed $(\mathrm{mm} / \mathrm{sec})$ & 44.5 & 18.7 & 2.38 & 0.028 & 22.67 \\
\hline layer Height $(\mathrm{mm})$ & 53.5 & 30.0 & 1.78 & 0.089 & 1.45 \\
\hline Energy Density $\left(\mathrm{J} / \mathrm{mm}^{2}\right)$ & 19.19 & 8.84 & 2.17 & 0.042 & 55.74 \\
\hline
\end{tabular}

Table 15: Analysis of Variance of UTS (MPa)

\begin{tabular}{|c|c|c|c|c|}
\hline Source & DF & Adj SS & Adj MS & F-Value \\
\hline Regression & 4 & 8402 & 2100.4 & 2.72 \\
\hline Power (W) & 1 & 3357 & 3356.8 & 4.35 \\
\hline Scanning Speed (mm/sec) & 1 & 4361 & 4361.2 & 5.65 \\
\hline layer Height (mm) & 1 & 2458 & 2458.4 & 3.19 \\
\hline Energy Density (J/mm $\left.{ }^{2}\right)$ & 1 & 3633 & 3632.6 & 4.71 \\
\hline Error & 20 & 15431 & 771.6 & \\
\hline Total & 24 & 23833 & & \\
\hline
\end{tabular}

\subsubsection{Regression Analysis: Yield Strength (MPa) versus Power (W), Scanning Speed $\underline{\mathrm{mm} / \mathrm{sec}) \text {, Height }(\mathrm{mm}) \text {, Energy Density }\left(\mathrm{J} / \mathrm{mm}^{2}\right)}$}

\section{Regression Equation}

Yield Strength $=193-0.196$ Power $(\mathrm{W})+12.2$ Scanning Speed $(\mathrm{mm} / \mathrm{sec})$

(MPa) - 2.7 Height $(\mathrm{mm})+7.47$ Energy Density $(\mathrm{J} / \mathrm{mm} 2)$

Table 16: Coefficients of regression analysis of Yield Strength (MPa)

\begin{tabular}{|c|c|c|c|c|c|}
\hline Term & Coef & SE Coef & T-Value & P-Value & VIF \\
\hline Constant & 193 & 111 & 1.74 & 0.098 & \\
\hline Power $(\mathrm{W})$ & -0.196 & 0.133 & -1.47 & 0.157 & 34.01 \\
\hline Scanning Speed (mm/sec) & 12.2 & 10.9 & 1.12 & 0.274 & 22.67 \\
\hline Height (mm) & -2.7 & 17.4 & -0.16 & 0.877 & 1.45 \\
\hline
\end{tabular}




\begin{tabular}{|l|l|l|l|l|l|}
\hline Energy Density $\left(\mathrm{J} / \mathrm{mm}^{2}\right)$ & 7.47 & 5.14 & 1.45 & 0.162 & 55.74 \\
\hline
\end{tabular}

Table 17: Analysis of Variance of Yield Strength (MPa)

\begin{tabular}{|c|c|c|c|c|}
\hline Source & DF & Adj SS & Adj MS & F-Value \\
\hline Regression & 4 & 1079.30 & 269.825 & 1.03 \\
\hline Power $(\mathrm{W})$ & 1 & 564.62 & 564.623 & 2.16 \\
\hline Scanning Speed $(\mathrm{mm} / \mathrm{sec})$ & 1 & 329.50 & 329.502 & 1.26 \\
\hline Height $(\mathrm{mm})$ & 1 & 6.43 & 6.429 & 0.02 \\
\hline Energy Density $\left(\mathrm{J} / \mathrm{mm}^{2}\right)$ & 1 & 550.76 & 550.760 & 2.11 \\
\hline Error & 20 & 5219.15 & 260.957 & \\
\hline Total & 24 & 6298.45 & & \\
\hline
\end{tabular}

\subsubsection{Observation}

The linear relationship between two or more variables is referred as multicollinearity. This is a kind of data related difficulty which may causes the reliability issues of the parameter estimators of the model. (Aylin Alin, 2010) Though multicollinearity affects the regression model and the relationship between the response and explanatory variables, it doesn't affect the model's significance. (Aylin Alin, 2010). It's possible to attain a significant model even if all the variables are insignificant. (David A. Belsley, 2014). There is a difference between correlation and collinearity. Therefore, collinearity may exist even all the correlations are lower. (Chatterjee et. al, 2013). Determinant of $\mathrm{R}$ is a good way to detect multicollinearity, but it also faces the difficulties with pairwise correlation of the variables. Despite being close to 1, there still possibility of multicollinearity among the explanatory variables. (Chatterjee et. al, 2013). In this case, variance inflation factor or VIF is a very popular and useful diagnostic means for multicollinearity.

From regression coefficients, the VIF values obtained for Laser power is 34.01, for scanning speed is 22.67 , layer height is 1.45 , for energy density is 55.74. The most used threshold value for VIF value is usually 10. (Aylin Alin, 2010). The T values we obtained are very smaller. Almost all the $P$ values we obtained are higher than the level of significance, $\alpha=0.05$, except for ultimate tensile strength, the $\mathrm{P}$ value for laser power (0.05), scanning speed (0.028) and energy density (0.042). These are clear indications of existence of multicollinearity which might have affected the variables being nonsignificant here. This also proves there exist no simple way to represent the linear relationship between the variables with the response of our data.

Multicollinearity can be of two types: data related where the data can't be manipulated which might be our case and structure related where the new variables are considered. (Stephanie Glan, 2015) Another reason of the multicollinearity existing in our experiment is the smaller data size. 
We could only perform 25 experiments with 5 groups of specimens. To avoid the multicollinearity related difficulties, we employed Ridge Regression to our problem.

\subsubsection{Ridge Regression}

Ridge regression is a suitable choice for data set with multicollinearity. If there is a possibility of multicollinearity, the variance of the lease square estimates gets too large to be closer to the true value though the estimates are unbiased. (McDonald et. al, 2009) The ridge regression equation as follows (Chapter 335, NCSS):

$$
\boldsymbol{Y}=\boldsymbol{B} \boldsymbol{X}+\boldsymbol{e}
$$

Here, $\mathrm{Y}$ denotes dependent variable, $\mathrm{X}$ denotes independent variable, $\mathrm{B}$ represents regression coefficient to be estimated, e denotes residuals/errors.

In ridge regression, standardization of both dependable and independent variables is a must. Standardization is basically subtracting the mean and then dividing by the standard deviation. Ridge regression is calculated on standardized variable. But the final regression co-efficient are estimated, they are adjusted back to the original scale. However, the ridge trace which will be discussed in later section is in standardized scale.

Ridge Trace: Ridge trace is represented by $\mathrm{k}$. The main challenge of ridge regression is selection of appropriate value of $\mathrm{k}$. Horel et. al, 1970, described ridge regression using a graph called ridge trace where ridge regression coefficients are displayed as a function of $\mathrm{k}$. First the value of $\mathrm{k}$ is selected then the regression coefficients get stabilized. But in most of the cases, the regression coefficient varies for different values of k. So, it's recommended to select smaller values of k. For smaller value of $\mathrm{k}$ which introduces smaller biases, the coefficients tend to stay constant. With the rise of the values of k, the coefficients reach to zero. (Michel Friendly, 2012)

From least square formulae, the regression coefficients are estimated using the following equation (Chapter 335, NCSS):

$$
B=\left(X^{\prime} X\right)^{-1} X^{\prime} Y
$$

Here we take $\boldsymbol{X}^{\prime} \boldsymbol{X}=\boldsymbol{R}$, which is a correlation matrix of independent variables. The estimates are unbiased. The expected values of the estimates are

$$
E(B)=\widehat{B}
$$

The variance-covariance matrix of the coefficients

And we assumed $\mathrm{Y}$ is standardized, so $\sigma^{2}=1$,

$$
V(B)=\sigma^{2} R^{-1}
$$

So, we found from the above equations,

$$
V\left(b_{j}\right)=r_{j j}=\frac{1}{1-R_{j}^{2}}
$$

$\boldsymbol{R}_{j}^{2}$ is the $\boldsymbol{R}$ squared value we obtained from the $\mathrm{X}_{\mathrm{j}}$ variables. Here the variance is the VIF we obtained.

The ridge regression proceeds with 


$$
V(B)=(R+k I)^{-1} X^{\prime} Y
$$

Here, $\boldsymbol{k}$ is the ridge trace which is a positive number less than 1 .

Now, the amount of bias from the estimators is following

$$
E(B-\widehat{B})=\left[\left(X^{\prime} X+k I\right)^{-1} X^{\prime} X-I\right] B
$$

The covariance matrix becomes,

$$
V(B)=\left[\left(X^{\prime} X+k I\right)^{-1} X^{\prime} X\left(X^{\prime} X+k I\right)^{-1}\right]
$$

We followed the same structure during building the Ridge Regression model. The data was first standardized by using MinMaxScaler() function called from Sklearn.preprocessing. For comparison purpose the test size kept $25 \%$ of the data using test_train_split. For the three tensile strength behavior. The predicted and test values of the three tensile properties are compared below:

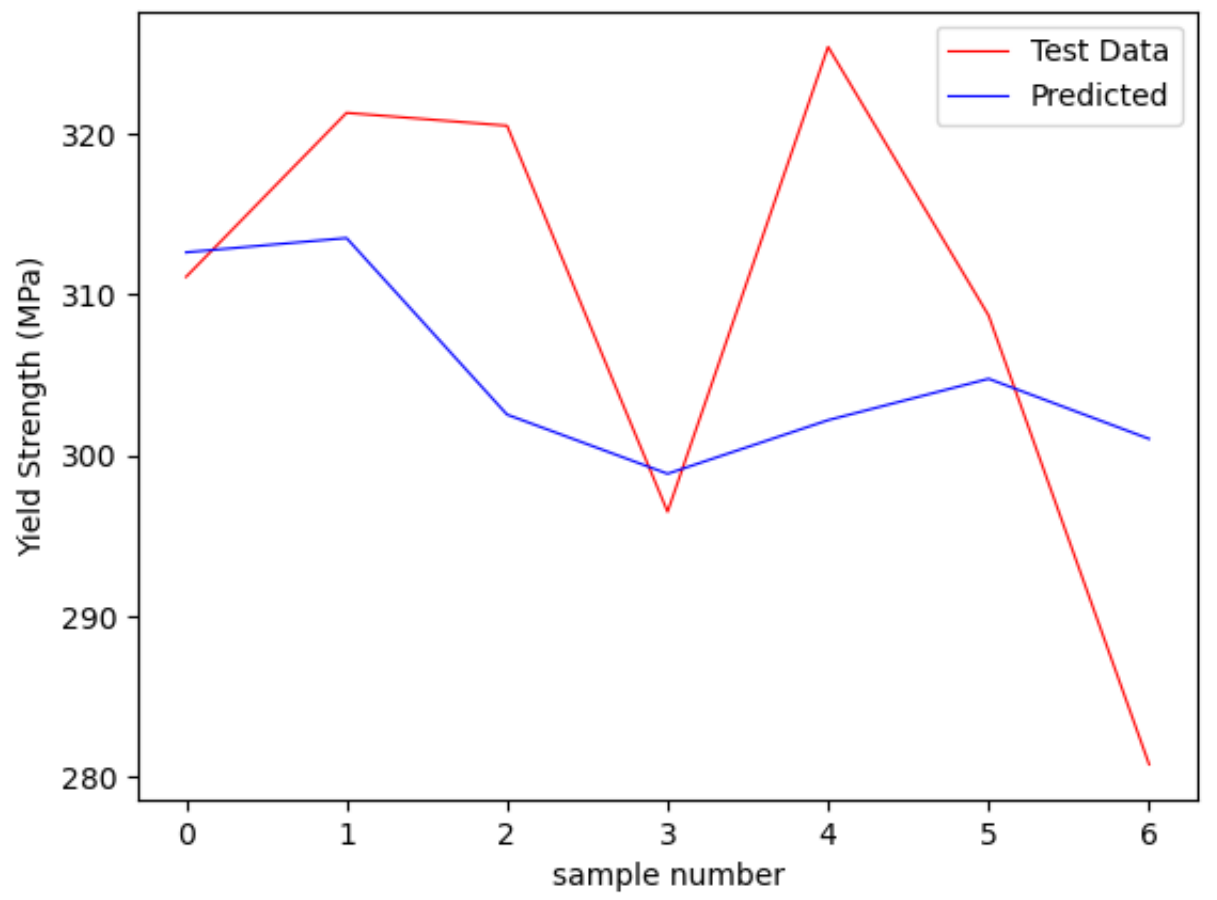

Figure 32: The predicted and test data for Yield Strength (MPa) by Ridge Regression. 


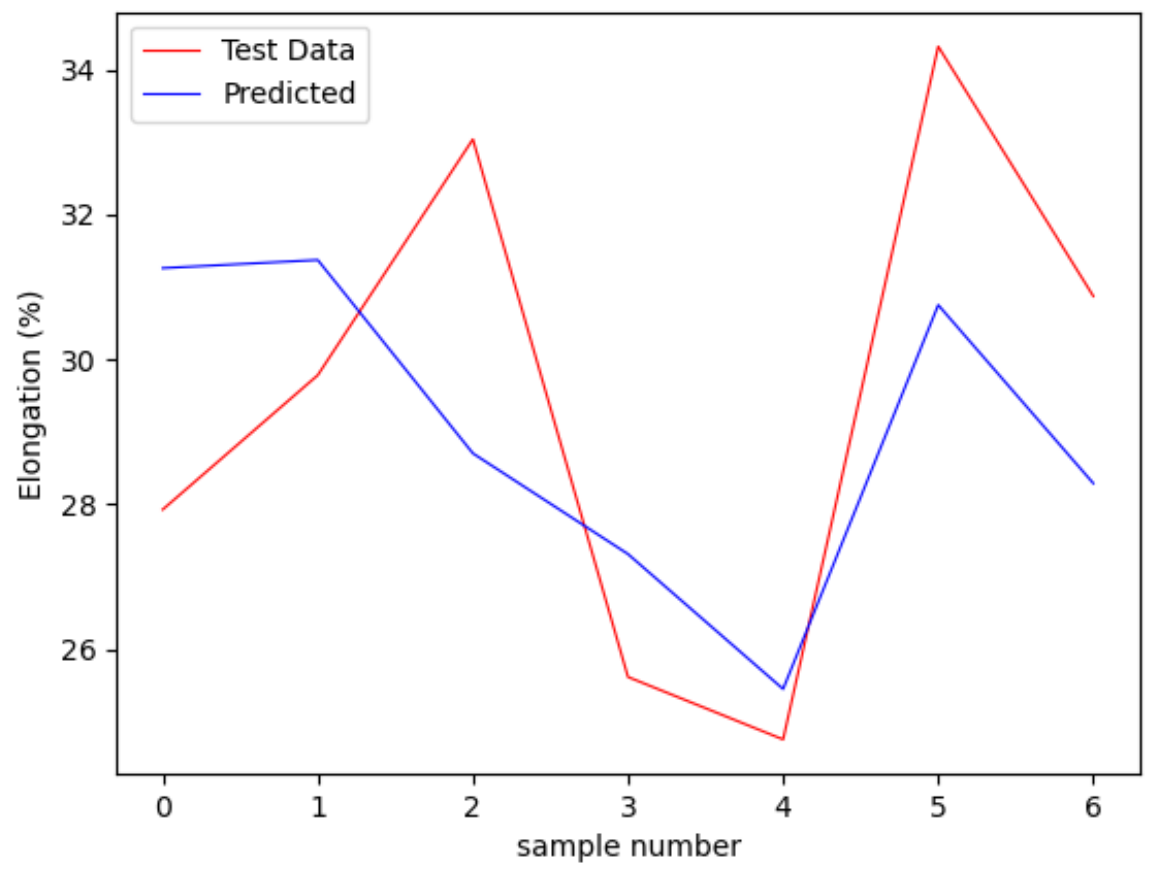

Figure 33: The predicted and test data for Elongation (\%) by Ridge Regression.

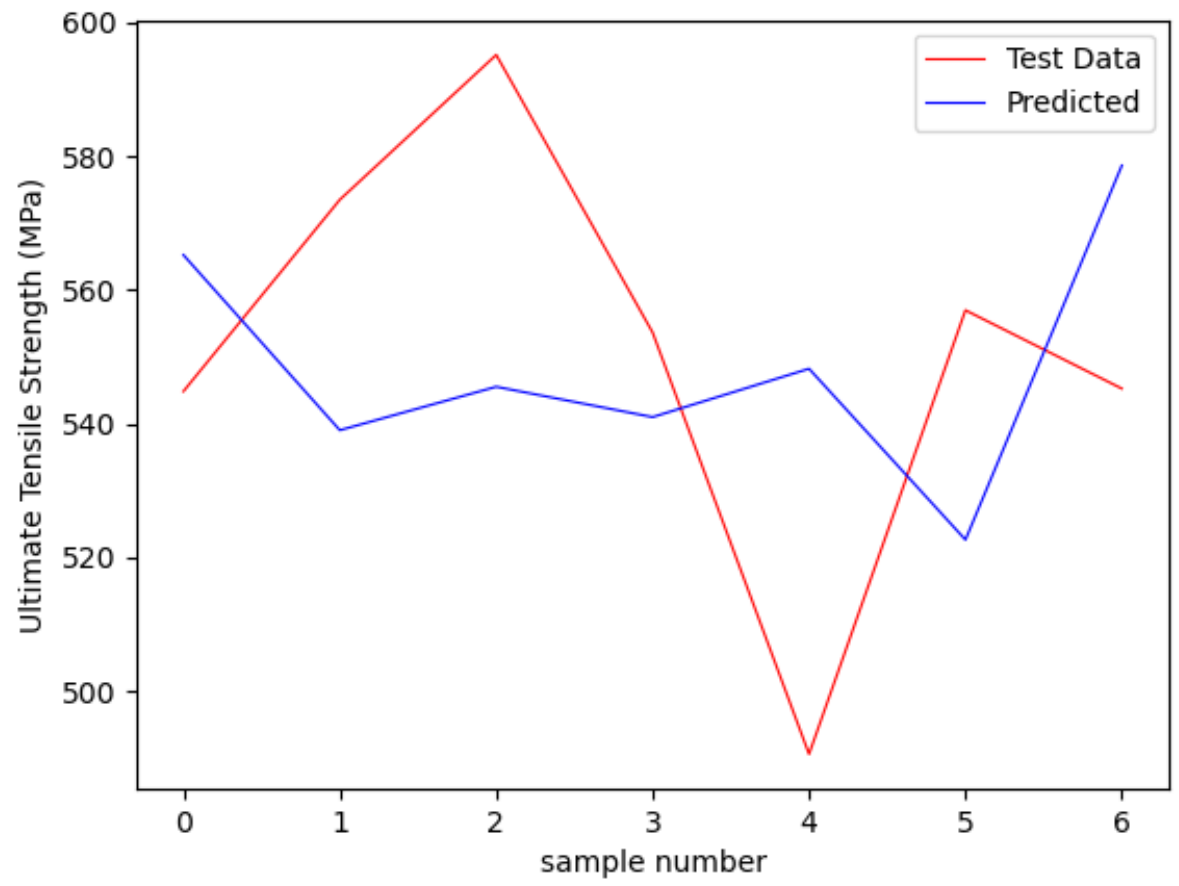

Figure 34: The predicted and test data for ultimate tensile strength (MPa) by Ridge Regression. 
Table 18: The performance evaluation metrics for Ridge Regression

\begin{tabular}{|c|c|c|c|}
\hline Test & RMSE & $\mathbf{R}^{2}$ & RE \\
\hline Yield Strength (MPa) & 21.89 & 0.49 & $2.93 \%$ \\
\hline Ultimate Tensile Strength (MPa) & 33.25 & 0.56 & $4.61 \%$ \\
\hline Elongation (\%) & 4.59 & 0.58 & $1.89 \%$ \\
\hline
\end{tabular}

From Table 18, The RMSE values obtained for the three outputs meant deviation of the predicted values from the test data. Similar to XGBoost, the deviation is highest for ultimate tensile strength (MPa) (33.25) and lowest for the elongation (\%) (4.59), which represented how much the model fitted to the training data of ultimate tensile strength (MPa) and elongation (\%). Coefficient of determination $\mathrm{R}^{2}$ values denoted is the model fitted to the $49 \%$ of the yield strength (MPa) data, $56 \%$ of the ultimate tensile strength (MPa) data and $58 \%$ of the elongation (\%) data. From the relative errors results, out take is that the relative uncertainty of the prediction to the actual values for yield strength is $2.93 \%$, for ultimate tensile strength (MPa) is $4.61 \%$ and for elongation (\%) is $1.89 \%$. The overall performance accuracy of the model is better but still didn't surpass the XGBoost model.

\subsection{Discussion}

Comparing the evaluation metrics form the Tables 11 and 18, the performance accuracy of XGBoost in terms of RMSE for yield strength improved $72.17 \%$, for ultimate tensile strength $64.24 \%$, for elongation (\%) $42.48 \%$ compared to ridge regression. Coefficient of determination, $\mathrm{R}^{2}$ has improved for yield strength $45 \%$, for ultimate tensile strength $15.56 \%$ and for elongation (\%) $25.2 \%$ compared to ridge regression. Lastly in terms of relative error, the performance improved for yield strength $62.1 \%$, for ultimate tensile strength $29.93 \%$ and for elongation (\%) $52.5 \%$ compared to ridge regression.

Although the internal mechanism of the algorithm is not easy to interpret, XGBoost still is a better choice for prediction problems. The basic mechanism which makes XGBoost faster is the weights are calculated as the ratio of the square of the sum of the residuals to the sum of the number of residuals and the regularization factor whereas for Ridge Regression, it's the sum of the squared residuals in the denominator. So, in case of XGBoost, the sum of the residuals first neutralizes the negative values which reduces the overall values of residual. On the other hand, the squared values of the residuals in Ridge Regression are always non-negative which increases the values of residuals for ridge compared to XGBoost.

Moreover, XGBoost possess both reproducibility and replicability characteristics. Reproducibility in terms of machine learning is a characteristic of a model which let it to obtain similar results on 
the same data set if applied several times. (McDonnel et. al, 2019) Replicability is the feature which let the model obtain closer result to the original one on different data set. (McDonnel et. al, 2019) The first model that we have built to verify the prediction quality on the same molten pool data with the work of Zhang et. al, 2020, showed almost similar outcome with very slight deviation from the published work. If we recall the RMSE values, in first case the RMSE of our model improved by $2.08 \%$ from the Zhang et. al, 2020. In second case, the original model improved $40 \%$ than our model. In case three our model scored exactly same RMSE value and in fourth case, the original model beat our model with $25 \%$ improvement. Our model was able to reproduce the results with very high accuracy. We used the similar model architecture except for few customizations for the tensile properties data to generate similar performance to get advantage of replicability as well.

Except for ensemble models XGBoost proved to outperform deep learning and other vector machine learning methods when it comes to smaller size of tabular data. (Shwartz-Ziv et. al, 202). Ridge regression, Lasso regression, XGBoost and the ensemble model of all three of them were performed on Ames Housing data set on Kaggle competition of which the author claimed is with huge multicollinearity. (Nasser Bashkeel, 2018). The RMSE values are used as the performance evaluation where Ridge regression came first with 0.1125 and XGboost came with 0.1230 . The ensemble learning had the RMSE equal to 0.1220. According to Shwartz-Ziv et. al, 2021, XGBoost performed the second best with 3.4\% accuracy compared to the $1 \mathrm{D}-\mathrm{CNN}$ with $7.5 \%$, TabNet with $10.5 \%$, DNF-Net with $11.7 \%$, NODE with $14.2 \%$ but the ensemble of all these models scored the best performance with $2.32 \%$ average relative increase. In our work the same outcome reflected by XGBoost outperforming ridge regression compared to the evaluation metrics of ridge regression.

\subsection{Limitations}

The multicollinearity observed in the data could be another reason the models might have suffered to predict the tensile properties with better accuracy. Even for XGBoost which is capable to fit small size of tabular data, our data is insufficient for the model to train. The experimentation includes three extensive lab work i.e. fabrication, preparation of the final specimens by EDM and finally the tensile testing. Fabrication by the L-DED machine is very time consuming and expensive. Due to time and facility constraints due to COVID 19, EDM facilities weren't available to the users. For future work, more experimentations are recommended to perform to deal with multicollinearity and to improve the performance accuracy of the model.

Variation in heat affected zones (HAZ) on the substrate is observed in Figure 10. Specimens printed with higher laser power have more HAZ compared to ones with lower laser power. In Group 3, 4 layers are printed instead of 5 layers because scanning speed is the lowest and more powder is required. To print 5 layers, the difference of heights between the deposition head and the top layer becomes $5 \mathrm{~mm}$ which should be at least $10 \mathrm{~mm}$. This may cause severe damage to the deposition head.

The height of specimen with lower laser power is comparatively lower than the specimens printed at higher laser power. This happened because laser power affects the powder efficiency meaning to what extent the powder is getting melted. With more power, larger amount of powder gets melted, thus increasing the height of the specimen. 
Its already mentioned before DED process is prone to rough surface finish and internal porosity. (Gibson et. al, 2015). The stress-strain curve we have obtained are similar but not identical between any two specimens. This variation of tensile properties possibly resulted from the variation of the layer thickness (Shim et. al, 2016), which cause by the surface and internal defects of the fabricated specimens. According to Zheng et. al, 2019 the internal defects observed in 316 Stainless Steel specimens fabricated by L-DED process and these defects can be divided mainly into two kinds: gas porosity and lack-of-fusion porosity. The final specimens are cut from the original one with the substrate by electric discharge machining. The final thickness was preset to $2 \mathrm{~mm}$ for all the specimens, but the cutting process faced difficulties due to defects of the specimens. Therefore, the thickness couldn't be maintained constant to $2 \mathrm{~mm}$ for all the groups and after manual surface finishing the final layer height ranged from $0.79 \mathrm{~mm}$ to $1.83 \mathrm{~mm}$ with an average of $1.51 \mathrm{~mm}$.

It was observed that, the first two groups with higher energy density due to its lower scanning speeds $8 \mathrm{~mm} / \mathrm{sec}$ and $9 \mathrm{~mm} / \mathrm{sec}$ had more constant layer thickness and was closer to $2 \mathrm{~mm}$ while the deviation from $2 \mathrm{~mm}$ and the more variation in the layer height was observed in the rest of the groups as well. It can be concluded that with higher energy density which means lower scanning speed, there are less defects like entrapped gas porosity and un-melted particles found in the specimen.

From Figure 23, 24 and 25, feature importance from XGBoost model, layer height attained the highest $\mathrm{F}$ score in both cases of yield strength and ultimate tensile strength, which means controlling layer heights of the specimen will yield better results for both the tensile strengths for 316 SS parts. Energy density came second in terms of F score for both yield and ultimate tensile strength. This means the combined effect of laser power and scanning speed have a control over the tensile properties of the specimens as well. It was observed just after the printing that with higher laser power, the powder efficiency increased. Though higher powder efficiency is responsible for the higher thickness of the original specimen, it is evident that it couldn't control the un-melted particles in the melt pool. 


\section{Conclusion \& Future Work}

\subsection{Conclusion}

The goal of this work is to employ machine learning to predict the tensile behavior of 316 Stainless Steel printed by laser assisted directed energy deposition process. The motivation behind this work is to examine the tensile properties of the printed specimens by L-DED process and at the same time to be able to predict the tensile behavior of the fabricated parts without performing the series of laborious experiments. The results from the tensile testing provided outstanding insight which exhibited better mechanical properties of the printed parts compared to raw 316 Stainless Steel. A total of 25 experiments with three sets of data have been performed and XGBoost has been selected because of its capability to predict with smaller tabular data. The model successfully predicted both the molten pool temperature which is used for verification of this model as well as the tensile properties of the fabricated parts which were collected experimentally. From this research work, it can be concluded:

- The yield strength and ultimate tensile strength quality of the printed 316 Stainless Steel were better than the raw materials.

- The XGBoost model was capable of both reproducibility in case of molten pool temperature data and replicability while performing on the tensile strength data.

- This model outperformed Ridge Regression and handled the multicollinearity better than the Ridge Regression model.

- Our study found from the most affecting parameters to the least affecting one for the tensile behaviors of the fabricated parts. Layer Heights for both yield (MPa) and ultimate tensile strength (MPa) and scanning speed for elongation (\%).

\subsection{Future Work}

We have faced few challenges and difficulties during the whole research which leaves us with the future prospects and forthcoming investigation of this study.

- We didn't take into account of the effects of thermal behavior and solidification on the print quality in our study. Due to varied laser power and additive layer nature of the process, the specimen experienced complex thermal cycles in the different location of the built. (Zheng et. al, 2019). Thermal behaviors can add to a great deal to the prediction quality of the model.

- According to (Zheng et. al, 2019), a traversed molten pool formed by the finely focused laser beam causes the higher cooling rate and solidification. These complex thermal behaviors are responsible for microstructure complexity due to which the consistency of the tensile properties might have suffered. So, microstructure can be used as one of the outcomes along with the tensile properties to establish the correlation among them.

- Furthermore, the inconsistency of the tensile properties of different groups also affected the learning curve of the model during training. So, we should be careful during data collection to avoid multicollinearity and other data related difficulties which worsen the prediction quality. 
- To improve the model's accuracy, different parameters should be considered along with the current ones in terms of $\mathrm{F}$ score to find out the optimum combination of parameters affecting the results. Based on feature importance, more experiments should be performed while modeling to examine whether the model performs better with and without the most important factors.

- Moreover, more experiments should be performed to enlarge the data set, at least to 100 samples, to overcome the multicollinearity and to improve the prediction accuracy as well. 


\section{$\underline{\text { References }}$}

[1] "ASTM52900-15 Standard Terminology for Additive Manufacturing-General PrinciplesTerminology." doi:10.1520/f3177-15.

[2] Aarshay JainAarshay. XGBoost Parameters: XGBoost Parameter Tuning. 23 Nov. 2020, www.analyticsvidhya.com/blog/2016/03/complete-guide-parameter-tuning-xgboost-withcodes-python/.

[3] Alin, Aylin. "Multicollinearity." Wiley Interdisciplinary Reviews: Computational Statistics, vol. 2, no. 3, 2010, pp. 370-374.

[4] Bashkeel. "EDA to Ensemble Model: Lasso + Ridge + XGBoost." Kaggle, Kaggle, 30 Oct. 2018, www.kaggle.com/bashkeel/eda-to-ensemble-model-lasso-ridge-xgboost.

[5] Belsley, David A. "Conditioning Diagnostics." Wiley StatsRef: Statistics Reference Online, 2014.

[6] Benardos, P.G, and G.C Vosniakos. "Prediction of Surface Roughness in CNC Face Milling Using Neural Networks and Taguchi's Design of Experiments." Robotics and ComputerIntegrated Manufacturing, vol. 18, no. 5-6, 2002, pp. 343-354., doi:10.1016/s07365845(02)00005-4.

[7] Bouckaert, Remco R., and Eibe Frank. "Evaluating the Replicability of Significance Tests for Comparing Learning Algorithms." Advances in Knowledge Discovery and Data Mining, 2004, pp. 3-12.

[8] Brownlee, Jason. "A Gentle Introduction to XGBoost for Applied Machine Learning." Machine Learning Mastery, 16 Feb. 2021, machinelearningmastery.com/gentle-introductionxgboost-applied-machine-learning/.

[9] Caiazzo, Fabrizia, and Alessandra Caggiano. "Laser Direct Metal Deposition of 2024 Al Alloy: Trace Geometry Prediction via Machine Learning." Materials, vol. 11, no. 3, 2018, p. 444., doi:10.3390/ma11030444.

[10] Cao, Lin, et al. "Effect of Laser Energy Density on Defects Behavior of Direct Laser Depositing 24CrNiMo Alloy Steel.” Optics \& Laser Technology, vol. 111, 2019, pp. 541-553., doi:10.1016/j.optlastec.2018.10.025.

[11] Chapter 335 Ridge Regression - NCSS. ncss-wpengine.netdna-ssl.com/wp content/themes/ncss/pdf/Procedures/NCSS/Ridge_Regression.pdf.

[12] Chatterjee, Samprit, and Ali S. Hadi. Regression Analysis by Example. Wiley, 2013.

[13] Chen, Tianqi, and Carlos Guestrin. "XGBoost." Proceedings of the 22nd ACM SIGKDD International Conference on Knowledge Discovery and Data Mining, 2016, doi:10.1145/2939672.2939785.

[14] Chen, Yi-Wei, and Chih-Jen Lin. "Combining SVMs with Various Feature Selection Strategies." Feature Extraction, pp. 315-324., doi:10.1007/978-3-540-35488-8_13.

[15] Cortez, Paulo, and Mark J. Embrechts. "Using Sensitivity Analysis and Visualization Techniques to Open Black Box Data Mining Models." Information Sciences, vol. 225, 2013, pp. 1-17., doi:10.1016/j.ins.2012.10.039.

[16] Dass, Adrita, and Atieh Moridi. "State of the Art in Directed Energy Deposition: From Additive Manufacturing to Materials Design.” Coatings, vol. 9, no. 7, 2019, p. 418., doi:10.3390/coatings9070418.

[17] De La Batut, Benoit, et al. "Analytical and Numerical Temperature Prediction in Direct Metal Deposition of Ti6Al4V." Journal of Manufacturing and Materials Processing, vol. 1, no. 1, 2017, p. 3., doi:10.3390/jmmp1010003. 
[18] Farrar, Donald E, and Robert R Glauber. "MULTICOLLINEARITY IN REGRESSION ANALYSIS: THE PROBLEM REVISITED." The Review of Economics and Statistics, vol. 49, no. 1, 1969, pp. 92-107.

[19] Feng, Ji, et al. "Multi-Layered Gradient Boosting Decision Trees." NeurIPS 2018, 2018, doi:https://arxiv.org/abs/1806.00007.

[20] Friendly, Michael. "The Generalized Ridge Trace Plot: Visualizing Bias and Precision." Journal of Computational and Graphical Statistics, vol. 22, no. 1, 2012, pp. 50-68.

[21] Froes, F. H., and Rodney Boyer. Additive Manufacturing for the Aerospace Industry. Elsevier, 2019.

[22] Garg, Akhil, and K. Tai. "An Ensemble Approach of Machine Learning in Evaluation of Mechanical Property of the Rapid Prototyping Fabricated Prototype." Applied Mechanics and Materials, vol. 575, 2014, pp. 493-496., doi:10.4028/www.scientific.net/amm.575.493.

[23] Garg, Ashu, and Anirban Bhattacharya. "An Insight to the Failure of FDM Parts under Tensile Loading: Finite Element Analysis and Experimental Study." International Journal of Mechanical Sciences, vol. 120, 2017, pp. 225-236., doi:10.1016/j.ijmecsci.2016.11.032.

[24] Gibson, Ian, et al. "Additive Manufacturing Technologies.” 2015, doi:10.1007/978-1-49392113-3.

[25] Gao, Junfeng, et al. "A Novel Approach for Lie Detection Based on F-Score and Extreme Learning Machine." PLoS ONE, vol. 8, no. 6, 2013.

[26] Güneş, Salih, et al. "Multi-Class f-Score Feature Selection Approach to Classification of Obstructive Sleep Apnea Syndrome.” Expert Systems with Applications, vol. 37, no. 2, 2010, pp. 998-1004.

[27] Haitovsky, Yoel. "Multicollinearity in Regression Analysis: Comment." The Review of Economics and Statistics, vol. 51, no. 4, 1969, p. 486.

[28] Hansel, Adam, et al. "Study on Consistently Optimum Deposition Conditions of Typical Metal Material Using Additive/Subtractive Hybrid Machine Tool.” Procedia CIRP, vol. 46, 2016, pp. 579-582., doi:10.1016/j.procir.2016.04.113.

[29] Hayes, Brian J., et al. "Predicting Tensile Properties of Ti-6Al-4V Produced via Directed Energy Deposition." Acta Materialia, vol. 133, 2017, pp. 120-133., doi:10.1016/j.actamat.2017.05.025.

[30] "How to Control Your XGBoost Model." Capital One, www.capitalone.com/tech/machinelearning/how-to-control-your-xgboost-model/.

[31] Jesse Steinweg-Woods, Ph.D. A Guide to Gradient Boosted Trees with XGBoost in Python. jessesw.com/XG-Boost/.

[32] Jiang, Qiuhong, et al. "A Principal Component Analysis Based Three-Dimensional Sustainability Assessment Model to Evaluate Corporate Sustainable Performance." Journal of Cleaner Production, vol. 187, 2018, pp. 625-637., doi:10.1016/j.jclepro.2018.03.255.

[33] Keist, Jayme S., and Todd A. Palmer. "Role of Geometry on Properties of Additively Manufactured Ti-6Al-4V Structures Fabricated Using Laser Based Directed Energy Deposition." Materials \& Design, vol. 106, 2016, pp. 482-494., doi:10.1016/j.matdes.2016.05.045.

[34] Khairallah, Saad A., et al. "Laser Powder-Bed Fusion Additive Manufacturing: Physics of Complex Melt Flow and Formation Mechanisms of Pores, Spatter, and Denudation Zones." Acta Materialia, vol. 108, 2016, pp. 36-45., doi:10.1016/j.actamat.2016.02.014.

[35] Khalil, Yas, et al. "Influence of Laser Power on Tensile Properties and Material Characteristics of Laser-Sintered UHMWPE.” Manufacturing Review, vol. 3, 2016, p. 15. 
[36] Khanzadeh, Mojtaba, et al. "In-Situ Monitoring of Melt Pool Images for Porosity Prediction in Directed Energy Deposition Processes.” IISE Transactions, vol. 51, no. 5, 2018, pp. 437455., doi:10.1080/24725854.2017.1417656.

[37] Khanzadeh, Mojtaba, et al. "Porosity Prediction: Supervised-Learning of Thermal History for Direct Laser Deposition." Journal of Manufacturing Systems, vol. 47, 2018, pp. 69-82., doi:10.1016/j.jmsy.2018.04.001.

[38] Kumar, Kapil, and Sanjay Agarwal. "Multi-Objective Parametric Optimization on Machining with Wire Electric Discharge Machining." The International Journal of Advanced Manufacturing Technology, vol. 62, no. 5-8, 2011, pp. 617-633.

[39] Leon, Derczynski. "Complementarity, F-Score, and NLP Evaluation.” LREC (2016).

[40] Li, Zhixiong, et al. "Degradation Modeling and Remaining Useful Life Prediction of Aircraft Engines Using Ensemble Learning." Journal of Engineering for Gas Turbines and Power, vol. 141, no. 4, 2018, doi:10.1115/1.4041674.

[41] Li, Zhixiong, et al. "Prediction of Surface Roughness in Extrusion-Based Additive Manufacturing with Machine Learning." Robotics and Computer-Integrated Manufacturing, vol. 57, 2019, pp. 488-495., doi:10.1016/j.rcim.2019.01.004.

[42] Liao, Baoyi, et al. "Mathematical Model for Prediction and Optimization of Weld Bead Geometry in All-Position Automatic Welding of Pipes." Metals, vol. 8, no. 10, 2018, p. 756., doi:10.3390/met8100756.

[43] Liang, Pengfei, et al. "Machine Learning of Single-Cell Transcriptome Highly Identifies MRNA Signature by Comparing F-Score Selection with DGE Analysis." Molecular Therapy - Nucleic Acids, vol. 20, 2020, pp. 155-163.

[44] Maladkar, Kishan. Why Is Random Search Better Than Grid Search For Machine Learning. 10 Oct. 2020, analyticsindiamag.com/why-is-random-search-better-than-grid-search-formachine-learning/.

[45] Mazumder, Jyoti. "Design for Metallic Additive Manufacturing Machine with Capability for 'Certify as You Build."' Procedia CIRP, vol. 36, 2015, pp. 187-192., doi:10.1016/j.procir.2015.01.009.

[46] McDermott, Matthew B. A., et al. "Reproducibility in Machine Learning for Health." ArXiv.org, 2 July 2019, arxiv.org/abs/1907.01463.

[47] McDonald, Gary C. "Ridge Regression." Wiley Interdisciplinary Reviews: Computational Statistics, vol. 1, no. 1, 2009, pp. 93-100.

[48] Melenka, Garrett W., et al. "Evaluation and Prediction of the Tensile Properties of Continuous Fiber-Reinforced 3D Printed Structures." Composite Structures, vol. 153, 2016, pp. 866-875., doi:10.1016/j.compstruct.2016.07.018.

[49] Montemor, M.F., et al. "The Role of Mo in the Chemical Composition and Semiconductive Behaviour of Oxide Films Formed on Stainless Steels.” Corrosion Science, vol. 41, no. 1, 1999, pp. 17-34., doi:10.1016/s0010-938x(98)00126-7.

[50] Mozaffar, Mojtaba, et al. "Data-Driven Prediction of the High-Dimensional Thermal History in Directed Energy Deposition Processes via Recurrent Neural Networks." Manufacturing Letters, vol. 18, 2018, pp. 35-39., doi:10.1016/j.mfglet.2018.10.002.

[51] Mysinski, Wojciech. "Power Supply Unit for an Electric Discharge Machine." 2008 13th International Power Electronics and Motion Control Conference, 2008.

[52] Natekin, Alexey, and Alois Knoll. "Gradient Boosting Machines, a Tutorial." Frontiers in Neurorobotics, vol. 7, 2013. 
[53] Onwubolu, Godfrey C., and Farzad Rayegani. "Characterization and Optimization of Mechanical Properties of ABS Parts Manufactured by the Fused Deposition Modelling Process." International Journal of Manufacturing Engineering, vol. 2014, 2014, pp. 1-13., doi: $10.1155 / 2014 / 598531$.

[54] Padmanaban, G., and V. Balasubramanian. "Optimization of Laser Beam Welding Process Parameters to Attain Maximum Tensile Strength in AZ31B Magnesium Alloy.” Optics \& Laser Technology, vol. 42, no. 8, 2010, pp. 1253-1260.

[55] Polat, Kemal, and Salih Güneş. "A New Feature Selection Method on Classification of Medical Datasets: Kernel F-Score Feature Selection.” Expert Systems with Applications, vol. 36, no. 7, 2009, pp. 10367-10373., doi:10.1016/j.eswa.2009.01.041.

[56] Rao, R. Venkata, and Dhiraj P. Rai. "Optimization of Fused Deposition Modeling Process Using Teaching-Learning-Based Optimization Algorithm." Engineering Science and Technology, an International Journal, vol. 19, no. 1, 2016, pp. 587-603., doi:10.1016/j.jestch.2015.09.008.

[57] Scime, Luke, and Jack Beuth. "Using Machine Learning to Identify in-Situ Melt Pool Signatures Indicative of Flaw Formation in a Laser Powder Bed Fusion Additive Manufacturing Process." Additive Manufacturing, vol. 25, 2019, pp. 151-165., doi:10.1016/j.addma.2018.11.010.

[58] Shah, Kamran, et al. "Effects of Melt Pool Variables and Process Parameters in Laser Direct Metal Deposition of Aerospace Alloys." Materials and Manufacturing Processes, vol. 25, no. 12, 2010, pp. 1372-1380., doi:10.1080/10426914.2010.480999.

[59] Shamsaei, Nima, et al. "An Overview of Direct Laser Deposition for Additive Manufacturing; Part II: Mechanical Behavior, Process Parameter Optimization and Control." Additive Manufacturing, vol. 8, 2015, pp. 12-35., doi:10.1016/j.addma.2015.07.002.

[60] Shikhar. The Recent Queen of ML Algorithms: XGBoost, and It's Future. 16 Oct. 2019, medium.com/analytics-vidhya/the-recent-queen-of-ai-algos-xgboost-and-its-future$22 \mathrm{~d} 6 \mathrm{df} 3 \mathrm{~cd} 206$.

[61] Shim, Do-Sik, et al. "Effect of Layer Thickness Setting on Deposition Characteristics in Direct Energy Deposition (DED) Process." Optics \& Laser Technology, vol. 86, 2016, pp. 69-78.

[62] Shwartz-Ziv, Ravid, and Amitai Armon. "Tabular Data: Deep Learning Is Not All You Need." ArXiv.org, 6 June 2021, arxiv.org/abs/2106.03253v1.

[63] Song, QingJun, et al. "Feature Selection Based on FDA and F-Score for Multi-Class Classification." Expert Systems with Applications, vol. 81, 2017, pp. 22-27.

[64] Stephanie. "Multicollinearity: Definition, Causes, Examples." Statistics How To, 16 Sept. 2020, www.statisticshowto.com/multicollinearity/.

[65] "Tensile Test Experiment." Michigan Technological University, www.mtu.edu/materials/k12/experiments/tensile/.

[66] Wolff, Sarah J., et al. "Experimentally Validated Predictions of Thermal History and Microhardness in Laser-Deposited Inconel 718 on Carbon Steel." Additive Manufacturing, vol. 27, 2019, pp. 540-551., doi:10.1016/j.addma.2019.03.019.

[67] Wu, Dazhong, et al. "A Comparative Study on Machine Learning Algorithms for Smart Manufacturing: Tool Wear Prediction Using Random Forests." Journal of Manufacturing Science and Engineering, vol. 139, no. 7, 2017, doi:10.1115/1.4036350.

[68] Wu, Dazhong, et al. "Predictive Modelling of Surface Roughness in Fused Deposition Modelling Using Data Fusion.” International Journal of Production Research, vol. 57, no. 12, 2018, pp. 3992-4006., doi:10.1080/00207543.2018.1505058. 
[69] Zare, Yasser, and Kyong Yop Rhee. "Tensile Strength Prediction of Carbon Nanotube Reinforced Composites by Expansion of Cross-Orthogonal Skeleton Structure.” Composites Part B: Engineering, vol. 161, 2019, pp. 601-607., doi:10.1016/j.compositesb.2019.01.001.

[70] Zhang, Jianjing, et al. "Deep Learning-Based Tensile Strength Prediction in Fused Deposition Modeling." Computers in Industry, vol. 107, 2019, pp. 11-21., doi:10.1016/j.compind.2019.01.011.

[71] Zhang, Ziyang, et al. "Data-Driven Predictive Modeling of Tensile Behavior of Parts Fabricated by Cooperative 3D Printing." Journal of Computing and Information Science in Engineering, vol. 20, no. 2, 2019, doi:10.1115/1.4045290.

[72] Zhang, Ziyang, et al. "Prediction of Melt Pool Temperature in Directed Energy Deposition Using Machine Learning." Additive Manufacturing, vol. 37, 2021, p. 101692. doi:10.1016/j.addma.2020.101692.

[73] Zheng, B., et al. "On the Evolution of Microstructure and Defect Control in 316L SS Components Fabricated via Directed Energy Deposition.” Materials Science and Engineering: $A$, vol. 764, 2019, p. 138243.

[74] Zhong, Chongliang, et al. "Microstructures and Tensile Properties of Inconel 718 Formed by High Deposition-Rate Laser Metal Deposition.” Journal of Laser Applications, vol. 28, no. 2, 2016, p. 022010., doi:10.2351/1.4943290. 